\title{
An Extension of the Poisson Summation Formula to a Semi-Direct Product
}

\author{
by \\ Jason Lind

\begin{abstract}
A thesis submitted to the Faculty of Graduate and Postdoctoral Affairs in partial fulfillment of the requirements for the degree of
\end{abstract}

\author{
Master of Science \\ in \\ Pure Mathematics
}

Carleton University

Ottawa, Ontario

(C)Copyright

2011, Jason Lind 
Library and Archives

Canada

Published Heritage Branch

395 Wellington Street Ottawa ON K1A ON4 Canada
Bibliothèque et

Archives Canada

Direction du

Patrimoine de l'édition

395 , rue Wellington

Ottawa ON K1A ON4

Canada
Your file Votre référence

ISBN: 978-0-494-81704-9

Our file Notre référence

ISBN: 978-0-494-81704-9
NOTICE:

The author has granted a nonexclusive license allowing Library and Archives Canada to reproduce, publish, archive, preserve, conserve, communicate to the public by telecommunication or on the Internet, loan, distribute and sell theses worldwide, for commercial or noncommercial purposes, in microform, paper, electronic and/or any other formats.

The author retains copyright ownership and moral rights in this thesis. Neither the thesis nor substantial extracts from it may be printed or otherwise reproduced without the author's permission.
AVIS:

L'auteur a accordé une licence non exclusive permettant à la Bibliothèque et Archives Canada de reproduire, publier, archiver, sauvegarder, conserver, transmettre au public par télécommunication ou par l'Internet, prêter, distribuer et vendre des thèses partout dans le monde, à des fins commerciales ou autres, sur support microforme, papier, électronique et/ou autres formats.

L'auteur conserve la propriété du droit d'auteur et des droits moraux qui protège cette thèse. $\mathrm{Ni}$ la thèse ni des extraits substantiels de celle-ci ne doivent être imprimés ou autrement reproduits sans son autorisation.
In compliance with the Canadian Privacy Act some supporting forms may have been removed from this thesis.

While these forms may be included in the document page count, their removal does not represent any loss of content from the thesis.
Conformément à la loi canadienne sur la protection de la vie privée, quelques formulaires secondaires ont été enlevés de cette thèse.

Bien que ces formulaires aient inclus dans la pagination, il n'y aura aucun contenu manquant.

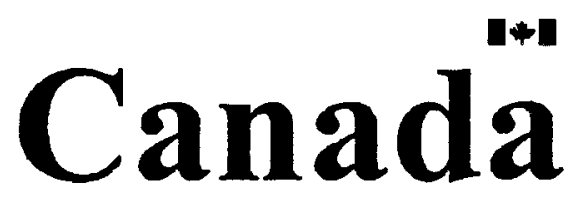




\section{Abstract}

Let $G$ be a second countable locally compact abelian group with a discrete subgroup $\Gamma$ such that the quotient space $G / \Gamma$ is compact. The Poisson Summation Formula states that for $f \in C_{c}(G)$, we have $\sum_{\gamma \in \Gamma} f(\gamma)=\sum_{\varphi \in \widehat{G / \Gamma}} \hat{f}(\varphi)$, whenever the sum on the right-hand side converges absolutely. Let $\Theta$ be a finite subgroup of $A u t(G)$ which preserves $\Gamma$. For a function $f \in C_{c}(G \rtimes \Theta)$, define the $\Gamma$-projection $f_{\Gamma} \in C(G / \Gamma \rtimes \Theta)$ by $f_{\Gamma}(x \Gamma, \theta)=\sum_{\gamma \in \Gamma} f(x \gamma, \theta)$ for all $(x \Gamma, \theta) \in G / \Gamma \rtimes \Theta$. Let $\overline{G / \Gamma \rtimes \Theta}$ denote the set of irreducible representations of $G / \Gamma \rtimes \Theta$ up to equivalence. For $f \in C_{c}(G \rtimes \Theta)$, we extend the Poisson Summation formula to $G / \Gamma \rtimes \Theta$ as

$$
\sum_{\gamma \in \Gamma} f\left(\gamma, e_{\Theta}\right)=\frac{1}{|\Theta|} \sum_{\pi \in G / \Gamma \times \Theta} \operatorname{deg}(\pi) \operatorname{tr} \int_{G / \Gamma} f_{\Gamma}\left(x \Gamma, e_{\Theta}\right) \pi\left(x^{-1} \Gamma, e_{\Theta}\right) d(x \Gamma)
$$

whenever the right-hand side converges absolutely, where $e_{\Theta}$ is the identity of $\Theta$. We make this formula explicit by classifying all $\pi \in G \widehat{/ \Gamma \rtimes} \Theta$ and computing their traces. 


\section{Acknowledgements}

First, I would like to thank my supervisor, Dr. Paul Mezo, for all the hard work he dedicated to this thesis. Thank you for keeping me on track and motivated throughout the process, as well as proving sound advice and encouragement. Without your continuous effort, this thesis would not be what it is today.

I would next like to thank all the other professors at Carleton University who have taught me everything I needed to know during my six years of undergrad and Masters studies. I would especially like to thank the efforts of Dr. Yuly Billig, Dr. Wojciech Jaworski and Dr. Benjamin Steinberg along with Dr. Paul Mezo in teaching me the fundamentals of topology, measure theory, functional analysis, group theory and representation theory. These subjects were at the core of my thesis and I would be nowhere without your sound teaching and guidance.

Finally, I would like to thank my mother Irene, my father Dennis, my brother Steve, my sister Emily and the countless other members of my family for always supporting me. Thanks to all my friends in mathematics for all the engaging discussions in the subject. A special thanks to Jay Crann for listening and helping in times where I was at a loss for ideas. Thanks to all my non-mathematical friends for your support. Even though you would be lost by the first sentence of the introduction of the thesis, you did your best to understand what I was working on. Thank You. 


\section{Contents}

$\begin{array}{ll}\text { Abstract } & \text { i }\end{array}$

1 Introduction $\quad 1$

2 Background Material $\quad 7$

2.1 Locally Compact Groups . . . . . . . . . . . . . . 7

2.2 Density of Characters of a Compact Group . . . . . . . . . . . . 12

3 The Poisson Summation Formula $\quad 21$

3.1 Invariant Measure On The Quotient Space . . . . . . . . . . 21

3.2 The Poisson Summation Formula . . . . . . . . . . . . 28

4 Traces of Linear Operators on $L^{2}(G / \Gamma) \quad 33$

4.1 The Regular Representation of $G / \Gamma \ldots \ldots 34$

4.2 A Related Representation of $G \ldots \ldots 43$

5 Traces of Linear Operators on $L^{2}(G / \Gamma \rtimes \Theta) \quad 47$

5.1 An Extension of $G / \Gamma$ using a Semi-Direct Product . . . . . . . . . 48

5.2 The Representation $R$ of $G \rtimes \Theta \ldots \ldots$. . . . . . . . . . 52

5.3 Computing the Integral Kernel . . . . . . . . . . . 55

5.4 The Right Regular Representation of $G / \Gamma \rtimes \Theta \ldots \ldots$

5.5 Computing the Trace of $R_{f} \ldots \ldots \ldots \ldots$. . . . . . . . 66 
6 Irreducible Representations of $G / \Gamma \rtimes \Theta$

6.1 A Group Action of $\Theta$ on $\widehat{G / \Gamma} \ldots \ldots$. . . . . . . . . . . 70

6.2 Induced Representations and Mackey's Irreducibility Criterion . . . . 73

6.3 The Classification of all Irreducible Representations of $G / \Gamma \times \Theta$. . 79

6.4 Computing the Traces of the Irreducible Representations . . . . . . 86

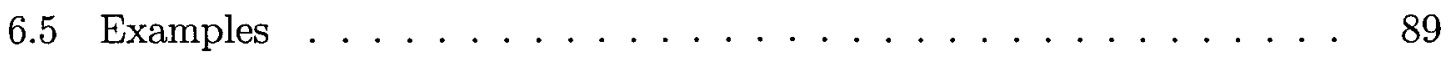

7 Extension of the Poisson Summation Formula $\quad 93$

7.1 Computing the Trace of a Linear Operator on $W_{j, \rho} \ldots \ldots$. . . . 94

7.2 The Peter-Weyl Theorem . . . . . . . . . . . . . . . . 96

7.3 Extending the Poisson Summation Formula to $G \rtimes \Theta \ldots \ldots 101$

7.4 The Independence of the Traces from $\Theta \ldots \ldots$. . . . . . . . 105

7.5 An Example on $\mathbb{Z}_{3} \ldots \ldots \ldots \ldots$. . . . . . . . . . . . 111

8 Conclusion $\quad 114$

$\begin{array}{ll}\text { Bibliography } & 115\end{array}$ 


\section{Chapter 1}

\section{Introduction}

For a topological space $X$, let $C_{c}(X)$ denote the space of continuous functions on $X$ with compact support. For $f \in C_{c}(\mathbb{R})$, the classical Poisson Summation Formula states that

$$
\sum_{n \in \mathbb{Z}} f(n)=\sum_{k \in \mathbb{Z}} \hat{f}(k)
$$

holds whenever the right-hand side converges absolutely. The function $\hat{f}: \mathbb{R} \rightarrow \mathbb{C}$ is the Fourier transform of $f$ defined by

$$
\widehat{f}(k)=\int_{-\infty}^{\infty} f(x) e^{-2 \pi \imath k x} d x
$$

for all $k \in \mathbb{R}$. Equivalently, one may define the Fourier transform $\hat{f}$ as a function on the group of continuous characters $\widehat{\mathbb{R}}$ of $\mathbb{R}$. The group $\widehat{\mathbb{R}}$ turns out to be isomorphic to $\mathbb{R}$ via the correspondence $k \leftrightarrow e^{2 \pi \imath k(\cdot)}$. This is why we have an equivalent definition. For a function $f \in C_{c}(\mathbb{R})$, we define the $\mathbb{Z}$-projection of $f, f_{\mathbb{Z}}: \mathbb{R} / \mathbb{Z} \rightarrow \mathbb{C}$, by $f_{\mathbb{Z}}(x+\mathbb{Z})=\sum_{k \in \mathbb{Z}} f(x+k)$ for all $x+\mathbb{Z} \in \mathbb{R} / \mathbb{Z}$. In this notation, (1.1) can be restated as

$$
f_{\mathbb{Z}}(0+\mathbb{Z})=\sum_{k \in \mathbb{Z}} \int_{0}^{1} f_{\mathbb{Z}}(x+\mathbb{Z}) e^{-2 \pi \imath k x} d x
$$


In more generality, the Fourier Inversion Theorem on $\mathbb{R} / \mathbb{Z}([22], \S 5.1 .5)$ implies that

$$
f_{\mathbb{Z}}(y+\mathbb{Z})=\sum_{k \in \mathbb{Z}} \hat{f}_{\mathbb{Z}}(k) e^{2 \pi \imath k y}=\sum_{k \in \mathbb{Z}}\left(\int_{0}^{1} f_{\mathbb{Z}}(x+\mathbb{Z}) e^{-2 \pi \imath k x} d x\right) e^{2 \pi \imath k y}
$$

holds true for every $y+\mathbb{Z} \in \mathbb{R} / \mathbb{Z}$ whenever the right-hand side converges absolutely. The Poisson Summation Formula follows from this implication of Fourier inversion by taking $y=0$. The original version of Poisson Summation Formula, as proved by Siméon Denis Poisson, had a much different language and was not proved using Fourier inversion on $\mathbb{R} / \mathbb{Z}([16])$. It wasn't until the early 20 th century that the Poisson Summation Formula began to take the form of (1.1) which we traditionally see today $([11]$, pg. 59).

The classical Poisson Summation Formula can be generalized to the theory of locally compact abelian groups. For a locally compact abelian group $H$, let $\hat{H}$ denote the set of continuous characters on $H$, and define the Fourier transform $\hat{f}$ of a function $f \in C_{c}(H)$ by

$$
\widehat{f}(\varphi)=\int_{H} f(x) \overline{\varphi(x)} d x
$$

for all $\varphi \in \hat{H}$, where integration is taken with respect to a fixed Haar measure on $H$. Consider a second countable locally compact abelian group $G$ with a discrete subgroup $\Gamma$ such that the quotient space $G / \Gamma$ is compact. As we shall see the group $\widehat{G / \Gamma}$ is discrete by the compactness of $G / \Gamma$. For $\varphi \in \widehat{G / \Gamma}$, let $\varphi^{\prime}$ be the character on $G$ given by $\varphi^{\prime}(x)=\varphi(x \Gamma)$ for all $x \in G$. For $f \in C_{c}(G)$, the Poisson Summation Formula is given by

$$
\sum_{\gamma \in \Gamma} f(\gamma)=\sum_{\varphi \in \widehat{G / \Gamma}} \hat{f}\left(\varphi^{\prime}\right)
$$


whenever the sum on the right-hand side is absolutely convergent. For $f \in C_{c}(G)$, we define the $\Gamma$-projection of $f, f_{\Gamma}: G / \Gamma \rightarrow \mathbb{C}$, by

$$
f_{\Gamma}(x \Gamma)=\sum_{\gamma \in \Gamma} f(x \gamma)
$$

As it happens, the $\Gamma$-projection of $f$ is a continuous function on $G / \Gamma$, and the Poisson Summation Formula can be restated as

$$
f_{\Gamma}(e \Gamma)=\sum_{\varphi \in \widehat{G / \Gamma}} \hat{f}_{\Gamma}(\varphi)
$$

whenever the right-hand side is absolutely convergent. With this approach, it suffices to look at continuous functions on the compact group $G / \Gamma$ in order to determine when the sum on the right-hand side converges absolutely. Although (1.2) is more general than the classical formula (1.1), this version of the Poisson Summation Formula is not much more difficult to prove. As with the classical formula, the proof follows from Fourier inversion on $G / \Gamma$, which implies that

$$
f_{\Gamma}(x \Gamma)=\sum_{\varphi \in \widehat{G / \Gamma}} \hat{f}_{\Gamma}(\varphi) \varphi(x \Gamma)
$$

holds true for all $x \Gamma \in G / \Gamma$ whenever the right-hand side converges absolutely.

It is proven in Hewitt and Ross ([9], Thm 34.5) that for a compact group $H$ and $g \in C_{c}(H)$, we have Fourier inversion on $H$ given by

$$
g(e)=\sum_{\pi \in \hat{H}} \operatorname{deg}(\pi) \operatorname{tr} \int_{H} g(x) \pi\left(x^{-1}\right) d x
$$

whenever the sum on the right converges absolutely. The space $\hat{H}$ is the complete set of all irreducible representations of $H$ (up to equivalence). Integration is taken with respect to a left Haar measure on $H$ that is normalized to have measure 1 on all of $H$, 
and is actually the integral of a matrix when $\operatorname{deg}(\pi)>1$. In this case of $\operatorname{deg}(\pi)>1$, we have that $\int_{H} g(x) \pi\left(x^{-1}\right) d x$ is a matrix defined by integrating the matrix coefficients, and the value $\operatorname{tr} \int_{H} g(x) \pi\left(x^{-1}\right) d x$ is its trace. The precise space of functions $g \in C_{c}(H)$ where the sum converges absolutely is proven to be $L^{2}(H) * L^{2}(H)([9]$, Thm 34.16), where * denotes the convolution of functions. When $H=G / \Gamma$ and $g=f_{\Gamma}$ for some $f \in C_{c}(G),(1.4)$ reduces to the generalized Poisson summation formula (1.3). This is because all irreducible representations of an abelian group have degree 1 , and $\hat{f}_{\Gamma}(\pi)=\int_{G / \Gamma} f_{\Gamma}(e \Gamma) \pi\left(x^{-1}\right) d x$ for every $\pi \in \widehat{G / \Gamma}$.

Returning to the general case of a compact group $H$ and $g \in C_{c}(H)$, we may rewrite the right-hand side of (1.4) in terms of a single representation $\bar{R}$ of $H$, called the right regular representation. Consider the bounded linear operator $\bar{R}_{g}$ on $L^{2}(H)$ given by

$$
\left(\bar{R}_{g} h\right)(y)=\int_{H} g(x)(\bar{R}(x) h)(y) d x=\int_{H} g(x) h(y x) d x
$$

where $g \in L^{2}(H)$ and $y \in H$. According to the Peter-Weyl Theorem, $\operatorname{tr}\left(\bar{R}_{g}\right)$ is equal to the right-hand side of (1.4). We call this side called the spectral side. The lefthand side of (1.4) is called the geometric side.

Our goal is to prove (1.4) in the case that $H=G / \Gamma \rtimes \Theta$, where $\Theta$ is a finite subgroup of $\operatorname{Aut}(G)$ which preserves $\Gamma$, and $g=f_{\Gamma}$, the $\Gamma$-projection of a function $f \in C_{c}(G \rtimes \Theta)$. The Poisson Summation Formula follows from Hewitt and Ross as above. However, as opposed to Hewitt and Ross, we prove (1.4) by computing $\operatorname{tr}\left(\bar{R}_{f_{\Gamma}}\right)$ using two separate methods. The first method is to prove that $\bar{R}_{f_{\Gamma}}$ is an integral operator. This is to say we prove the existence of a continuous function $K_{f_{\Gamma}} \in L^{2}((G / \Gamma \rtimes \Theta) \times(G / \Gamma \rtimes \Theta))$ such that

$$
\left(\bar{R}_{f_{\Gamma}} g\right)(y)=\int_{G / \Gamma \rtimes \Theta} K_{f_{\Gamma}}(y, x) g(x) d x
$$


for all $g \in L^{2}(G / \Gamma \rtimes \Theta)$ and $y \in G / \Gamma \rtimes \Theta$. The function $K_{f_{\Gamma}}$ is called the integral kernel of $\bar{R}_{f_{\Gamma}}$. It will be shown that $K_{f_{\Gamma}}(y, x)=f_{\Gamma}\left(y^{-1} x\right)$ for all $x, y \in G / \Gamma \rtimes \Theta$. According to Duflo ([4], Prop 3.3.1), the trace of $\bar{R}_{f_{\Gamma}}$ is given in terms of the integral kernel by

$$
\operatorname{tr}\left(\bar{R}_{f_{\Gamma}}\right)=\int_{G / \Gamma \rtimes \Theta} K_{f_{\Gamma}}(x, x) d x=|\Theta| f_{\Gamma}\left(e \Gamma, e_{\Theta}\right)
$$

whenever $\bar{R}_{f_{\Gamma}}$ is a trace class operator. The value of $|\Theta|$ in (1.5) comes from the fact that the measure we use on the compact group $G / \Gamma \rtimes \Theta$ is normalized to be $|\Theta|$ as opposed to 1 . This is the geometric computation for the trace of $\bar{R}_{f_{\Gamma}}$, and gives us the geometric side of (1.4). Chapter 5 is devoted to this geometric computation of the trace.

The second method involves the Peter-Weyl Theorem. As mentioned earlier, it follows from the Peter-Weyl Theorem that the trace of $\bar{R}_{f_{\Gamma}}$ is given by

$$
\operatorname{tr}\left(\bar{R}_{f_{\Gamma}}\right)=\sum_{\pi \in G / \Gamma \rtimes \Theta} \operatorname{deg}(\pi) \operatorname{tr} \int_{G / \Gamma \rtimes \Theta} f_{\Gamma}(x) \pi(x) d x
$$

whenever the sum on the right converges absolutely. Moreover, this sum converges absolutely if and only if $f_{\Gamma} \in L^{2}(G / \Gamma \rtimes \Theta) * L^{2}(G / \Gamma \rtimes \Theta)$. This is the spectral computation for the trace of $\bar{R}_{f_{\Gamma}}$, giving us the spectral side of (1.4). In Chapter 6, we will classify all irreducible representations of the group $G / \Gamma \rtimes \Theta$, which we have denoted here as elements of $\widehat{G / \Gamma \rtimes \Theta}$. Furthermore we compute their traces explicitly. In Chapter 7 , we determine the value of $\operatorname{tr} \int_{G / \Gamma \rtimes \Theta} f_{\Gamma}(x) \pi(x) d x$.

The geometric and spectral computations are then combined to form an extended Poisson Summation Formula for the group $G \rtimes \Theta$ given by

$$
\sum_{\gamma \in \Gamma} f\left(\gamma, e_{\Theta}\right)=\frac{1}{|\Theta|} \sum_{\pi \in G / \Gamma \rtimes \Theta} \operatorname{deg}(\pi) \sum_{\theta \in \Theta} \int_{G / \Gamma} f_{\Gamma}(x \Gamma, \theta) \pi(x \Gamma, \theta) d(x \Gamma)
$$


whenever the sum on the right-hand side converges absolutely. We will prove that that this formula only depends on the values of $f_{\Gamma}$ at the identity of $\Theta$, meaning that

$$
\sum_{\gamma \in \Gamma} f\left(\gamma, e_{\Theta}\right)=\frac{1}{|\Theta|} \sum_{\pi \in G / \Gamma \rtimes \Theta} \operatorname{deg}(\pi) \int_{G / \Gamma} f_{\Gamma}\left(x \Gamma, e_{\Theta}\right) \pi\left(x \Gamma, e_{\Theta}\right) d(x \Gamma) .
$$

Throughout, we will follow a particular example where $G=\mathbb{Z}$ and $\Gamma=3 \mathbb{Z}$ in order to illustrate theorems and formulas. Another common example that we shall use is $G=\mathbb{R}$ and $\Gamma=\mathbb{Z}$, which gives us an extension of the classical Poisson Summation Formula.

The motivation for proving (1.4) in the case where $H=G / \Gamma \rtimes \Theta$ is to try to understand the trace formula for $\left(G L\left(2, \mathbb{A}_{\mathbb{F}}\right) \rtimes G a l(\mathbb{F} / \mathbb{Q})\right) / G L(2, \mathbb{F})$, where $\mathbb{F}$ is a finite Galois extension of $\mathbb{Q}$ and $\mathbb{A}_{\mathbb{F}}$ is the adele ring of $\mathbb{F}$. As opposed to the methods of proof given in Hewitt and Ross for (1.4), our methods can be carried over to $\left(G L\left(2, \mathbb{A}_{\mathbb{F}}\right) \rtimes G a l(\mathbb{F} / \mathbb{Q})\right) / G L(2, \mathbb{F})$, where $\left(G L\left(2, \mathbb{A}_{\mathbb{F}}\right) \rtimes G a l(\mathbb{F} / \mathbb{Q})\right) / G L(2, \mathbb{F})$ is neither a group (as $G L\left(2, \mathbb{F}\right.$ ) is not normal in $G L\left(2, \mathbb{A}_{\mathbb{F}}\right)$ ) nor compact. The relevance of a trace formula for $\left(G L\left(2, \mathbb{A}_{\mathbb{F}}\right) \rtimes G a l(F / \mathbb{Q})\right) / G L(2, \mathbb{F})$ lies far beyond the intentions of this thesis. The interested reader may refer to ([14]).

It is assumed that the reader is familiar with the fundamental concepts of measure theory, topology, functional analysis, group theory and representation theory for finite groups. As references that will contain all prerequisites, we recommend [3] for measure theory, [23] for topology, [2] for functional analysis, [5] for group theory and [19] for representation theory. A great reference for further study in Harmonic Analysis is [7], which covers many of the topics presented here. 


\section{Chapter 2}

\section{Background Material}

\subsection{Locally Compact Groups}

A topological group $H$ is both a topological space and group such that the group multiplication operation, $(x, y) \mapsto x y$, and the inverse operation, $x \mapsto x^{-1}$, are continuous maps. The stipulation that these two functions be continuous is incredibly valuable. It ensures that every right translation $x \mapsto y x$, where $y \in H$, is a homeomorphism of $H([15], \S 2.3)$. Thus, if we have a neighbourhood $U$ of the identity, the set $y U=\{y u: \quad u \in U\}$ will be a neighbourhood of $y$ which shares the same topological properties as $U$.

A subset $U$ of a topological group $H$ is called symmetric if $U=U^{-1}$, where $U^{-1}=\left\{u^{-1}: u \in U\right\}$. It is proven in $([3], \S 9.1)$ that for every open neighbourhood $U$ of the identity, there is a symmetric open neighbourhood $V$ of the identity such that $V$ is contained in $U$, and also an open neighbourhood $W$ of the identity such that $W^{2}=\left\{w_{1} w_{2}: w_{1}, w_{2} \in W\right\}$ is contained in $U$. These two types of open neighbourhoods of the identity will be used throughout this paper. 
A topological space is called locally compact if every element of the space has a neighbourhood base consisting of compact neighbourhoods. A locally compact group is a topological group which is locally compact and Hausdorff. To show that a topological group is a locally compact group, we only need to check that the identity of the group has a compact neighbourhood. For topological groups, being a Hausdorff space is equivalent to the set $\{e\}$ being closed, where $e$ is the identity of the group ([15], §2.3). If a locally compact group is abelian, we call it a locally compact abelian group, or LCA group. A compact group is a topological group which is compact and Hausdorff. Obviously, it is true that every compact group is also a locally compact group.

A topological group is called a discrete group if its topology is the discrete topology. Since every right translation is a homeomorphism, a topological group is discrete if and only if the set $\{e\}$ is open. A subgroup $S$ of a topological group $H$ is a topological group when equipped with the subspace topology inherited from $H,([15], \S 2.3)$ and is called a topological subgroup. A topological subgroup $D$ is called a discrete subgroup if the subspace topology on $D$ is discrete. Note that every group can be turned into a topological group by giving it the discrete topology. In this sense, as suggested in $([15], \S 2.3)$, the theory of groups is just a specific case of the theory of topological groups.

If $H$ is a topological group, and $N$ is a normal subgroup of $H$, then the quotient group $H / N$ is a topological group when equipped with the quotient topology with respect to the surjection $\pi: H \rightarrow H / N([15], \S 2.3)$.

\section{Examples:}

1. The real numbers $\mathbb{R}$, under addition with the usual topology, is a locally compact group. $\mathbb{Z}$ is a discrete subgroup of $\mathbb{R}$, and the quotient group $\mathbb{R} / \mathbb{Z}$ is a compact topological group. 
2. The non-zero complex numbers $\mathbb{C}^{\times}$, under multiplication with the usual topology, is a locally compact group. The set of all $z \in \mathbb{C}^{\times}$with $|z|=1$, denoted by $\mathbb{T}$, is a compact subgroup of $\mathbb{C}^{\times} . \mathbb{T}$ is called the circle group or the torus (more specifically the 1 -torus), and is homeomorphic to $\mathbb{R} / \mathbb{Z}$.

3. Any group $H$ which is given the discrete topology is locally compact, and is compact if and only if it is finite.

Let $H$ be a topological group, and $\mathcal{B}(H)$ be the $\sigma$-algebra generated by the open sets of $H$, called the Borel sets of $H$. A measure $\mu_{H}$ on $H$ is called a Radon measure if it is finite on compact sets, outer regular on all Borel sets of $H$, and inner regular on all open sets of $H$. The definitions of inner and outer regular can be found in ([3], §7.2). A non-zero Radon measure $\mu_{H}$ is called a left Haar measure if it is invariant under left translations, that is $\mu_{H}(x A)=\mu_{H}(A)$ for every $x \in H$ and every $A \in \mathcal{B}(H)$. Similarly $\mu_{H}$ is called a right Haar measure if it is invariant under right translations. Obviously, for abelian topological groups every left Haar measure is a right Haar measure. However, this may not be the case for a non-abelian group ([15], §2.5). Typically we will work with a left Haar measure. If a group has a left Haar measure which is also a right Haar measure, it is called unimodular. It is proven in $([7], \S 2.4)$ that compact groups are unimodular.

The existence and uniqueness of a (left) Haar measure for a locally compact group is proven in ([3], §9.2). A (left) Haar measure $\mu$ on a locally compact group $H$ is unique in the sense that for any other (left) Haar measure $\nu$ on $H$, there exists a positive real number $\alpha$ such that $\mu=\alpha \nu$. The existence of Haar measure is the main reason for working under the assumption of a locally compact group. As shown in the earlier examples, many common topological groups are locally compact, making this assumption a reasonable one. 
If we are more specific and assume a compact group, what more can be said about this Haar measure? In fact, it is proven in $([3], \S 9.3)$ that a (left) Haar measure on a locally compact group $H$ is finite if and only if $H$ is compact. This is an interesting result which will be useful later on when we are dealing with compact groups. When $H$ is a compact group, we typically normalize the (left) Haar measure so that $\mu_{H}(H)=1$.

\section{Examples:}

1. When $H=(\mathbb{R},+)$ under the usual topology on $\mathbb{R}$, Lebesgue measure $\lambda$ is invariant under left and right translations ([3], §1.4). That is, for any measurable set $A \subseteq \mathbb{R}$ and any $x \in \mathbb{R}$, we have $\lambda(x+A)=\lambda(A)$. Lebesgue measure is regular ([3], §1.4), and thus is finite on compact sets, inner regular on open sets and outer regular on all Borel sets. Therefore Lebesgue measure is a Radon measure. Note that the Lebesguemeasurable sets strictly contain the Borel sets $([3], \S 2.1)$. Thus the restriction of $\lambda$ to the Borel sets of $\mathbb{R}$ is a Haar measure on $\mathbb{R}$.

2. If $H$ is a finite group equipped with the discrete topology, then the counting measure $\mu_{H}$, given by $\mu_{H}(A)=|A|$ for all $A \subseteq H$, is clearly a Haar measure on $H$.

The following lemma about compact Haar measure on open sets will be used to prove the Fourier Inversion Theorem in $\S 3.2$ and Theorem 5.5.1 in $\S 5.5$.

Lemma 2.1.1. Let $H$ be a compact group with (left) Haar measure $\mu_{H}$. Then every open neighbourhood of each point in $H$ has non-zero measure with respect to $\mu_{H}$.

Proof: We will prove by contradiction. Suppose $x \in H$ and $U$ is an open neighbourhood of $e$ such that $\mu_{H}(x U)=0$. As $\mu_{H}$ is a Haar measure, for every $y \in H$, we have

$$
\mu_{H}(y U)=\mu_{H}\left(y x^{-1} x U\right)=\mu_{H}(x U)=0
$$


Thus, $y U$ is an open neighbourhood of $y$ with measure 0 . The set $\{y U: y \in H\}$ is an open cover of the compact space $H$, and thus there exists a finite subcover $y_{1} U, \ldots, y_{n} U \subseteq H$, meaning

$$
H=\cup_{\imath=1}^{n} y_{\imath} U .
$$

Therefore, we get that

$$
\begin{aligned}
\mu_{H}(H) & =\mu_{H}\left(\cup_{\imath=1}^{n} y_{\imath} U\right) \\
& \leqslant \sum_{\imath=1}^{n} \mu_{H}\left(y_{n} U\right) \\
& =0 .
\end{aligned}
$$

This is clearly a contradiction, since $\mu_{H}(H)$ is non-zero, since Haar measures are non-zero measures.

Note that Lemma 2.1 .1 will also be true when $H$ is more generally a locally compact group. The more general case could be proved in a similar fashion using a compact neighbourhood of non-zero measure. We will only need the lemma in the case of a compact group, which is why we only proved it for such a case.

Consider a locally compact group $H$ and a left Haar measure $\mu_{H}$ on $H$. We define an equivalence relation $\equiv$ on the set of functions $g: H \rightarrow \mathbb{C}$ by $g_{1} \equiv g_{2}$ if and only if $g_{1}$ and $g_{2}$ are equal $\mu_{H}$-almost everywhere. The space $L^{2}\left(H, \mu_{H}\right)$ is the set of all equivalence classes $[g]$ of Borel measurable functions $g: H \rightarrow \mathbb{C}$ which satisfy

$$
\sqrt{\int_{H} g(y) \overline{g(y)} d y}<\infty
$$

where the integral is taken with respect to the (left) Haar measure $\mu_{H}$. When it is understood which measure $\mu_{H}$ we are referring to, we usually denote the space as simply $L^{2}(H)$. Although the elements of $L^{2}(H)$ are actually equivalence classes of 
functions, we will customarily denote them by a representative of the equivalence class. The space $L^{2}(H)$ is a Hilbert space with respect to the $L^{2}$ inner product given by

$$
\left\langle g_{1}, g_{2}\right\rangle=\int_{H} g_{1}(y) \overline{g_{2}(y)} d y
$$

for all $g_{1}, g_{2} \in L^{2}(H)([3], \S 3.3)$. This inner product defines the $L^{2}$-norm on $L^{2}(H)$ given by $\|g\|_{2}=\sqrt{\langle g, g\rangle}$ for $g \in L^{2}(H)$.

The space $L^{1}\left(H, \mu_{H}\right)$ is the set of equivalence classes of Borel measurable functions $g: H \rightarrow \mathbb{C}$ which satisfy that

$$
\int_{H}|g(y)| d y<\infty
$$

where integration is taken with respect to $\mu_{H}$. Again, when $\mu_{H}$ is understood we denote the space as $L^{1}(H)$. Equivalently, $L^{1}(H)$ is called the space of $\mu_{H}$-integrable functions, or simply the integrable functions.

Note that integration with respect to a left Haar measure is invariant under a change of variables given by left multiplication. That is, if $g \in L^{1}(H)$ and $y \in H$, then

$$
\int_{H} g(y x) d x=\int_{H} g(x) d x
$$

\subsection{Density of Characters of a Compact Group}

In this section, we describe the continuous characters of a LCA group. The ultimate goal is to show that the group of continuous characters $\hat{H}$ of a compact group $H$ form a Hilbert space basis for $L^{2}(H)$. The proof of the Gelfand-Raikov Theorem will be excluded, as its proof is very long and requires higher-level functional analysis. 
We believe that the proof would detract the reader from the main goals of this section.

A character $\chi$ of an abelian group $H$ is a homomorphism from $H$ to $\mathbb{T}$, where $\mathbb{T}$ is the circle group (§2.1). For a LCA group $H$, we only consider characters which are continuous, and call the set of continuous characters, denoted $\hat{H}$, the dual group of $H$. This name is appropriate since $\hat{H}$ does form a group with group law $\varphi_{1} \varphi_{2}(h)=\varphi_{1}(h) \varphi_{2}(h)$, where $\varphi_{1}, \varphi_{2} \in \hat{H}$ and $h \in H$. The identity of this group is the trivial character $\varphi_{o}$, which is defined by $\varphi_{o}(h)=1$ for all $h \in H$, and inverses are given by $\varphi^{-1}(h)=\varphi\left(h^{-1}\right)=1 / \varphi(h)$ for all $h \in H$. Since the image of a character lies in the circle group, it holds that for all $\varphi \in \hat{H}$, we have $\varphi^{-1}=\bar{\varphi}$.

It is proven in $([23], \S 2.2)$ that a topology can be uniquely determined by its neighbourhood bases. We define a topology on $\hat{H}$, called the topology of uniform convergence on compact sets, by setting the neighbourhood base at $\varphi_{o}$ equal to the collection of sets of the form

$$
B\left(\varphi_{o}, \epsilon, K\right)=\left\{\varphi \in \hat{H}: \sup _{x \in K}\left|\varphi(x)-\varphi_{o}(x)\right|<\epsilon\right\}
$$

where $\epsilon>0$ and $K$ is a compact subset of $H$. Note that, by definition, these sets are open in this topological space, and that we get a neighbourhood base at the other characters of $\hat{H}$ by using the right translation homeomorphism.

Proposition 2.2.1. Suppose $H$ is a compact abelian group. Then the group $\hat{H}$, with the topology of uniform convergence on compact sets, is discrete.

Proof: Consider the set $B=B\left(\varphi_{0}, \epsilon, H\right)$, where $\varphi_{0}$ is the identity character and $\epsilon=\sqrt{2-\sqrt{2}} . \quad B$ is in the neighbourhood base of $\varphi_{0}$ mentioned previously, and is therefore an open set. 
Suppose $\varphi \in B$ with $\varphi \neq \varphi_{o}$. Since $\varphi$ is non-trivial, there exists $y \in H$ such that $\varphi(y) \neq 1$. Let $\varphi(y)=e^{2 \pi \imath \theta}$, where $\theta \in[-\pi, \pi)$. This is justified since the image of $\varphi$ is in $\mathbb{T}$.

As $|\varphi(y)-1|<\epsilon$ follows from our choice that $\varphi \in B$, we have that $\theta \in\left(-\frac{\pi}{4}, \frac{\pi}{4}\right)$ and $\theta \neq 0$. We will consider only the case $\theta \in\left(0, \frac{\pi}{4}\right)$, since the remaining case can be proved with a similar argument.

We can find a minimal positive integer $k$ such that $k \theta \in\left(\frac{\pi}{4}, \frac{\pi}{2}\right)$. Because we have $y^{k} \in H$, and $\varphi\left(y^{k}\right)=\varphi(y)^{k}=e^{2 \pi \imath k \theta}$, by our choice of $k$

$$
0<\cos (k \theta)<\frac{1}{\sqrt{2}}, \frac{1}{\sqrt{2}}<\sin (k \theta)<1
$$

This gives us that

$$
1>1-\cos (k \theta)>1-\frac{1}{\sqrt{2}}
$$

We have

$$
\sqrt{(1-\cos (k \theta))^{2}+(\sin (k \theta))^{2}}>\sqrt{\left(1-\frac{1}{\sqrt{2}}\right)^{2}+\left(\frac{1}{\sqrt{2}}\right)^{2}}=\epsilon
$$

which shows $\left|\varphi\left(y^{k}\right)-1\right|>\epsilon$. Thus $\varphi$ is not in $B$, which is a contradiction.

Therefore we have $B=\left\{\varphi_{o}\right\}$, which is open, proving $\hat{H}$ is discrete.

Proposition 2.2.1 has just shown that when $H$ is a compact abelian group, $\hat{H}$ is a locally compact group. This is because, as mentioned previously in an example, every group under the discrete topology is a locally compact group. 


\section{Examples:}

1. Let $H=\langle a\rangle$ be a finite cyclic group of order $n$ generated by $a \in H$, equipped with the discrete topology. The group of continuous characters $\hat{H}$ is the set $\left\{\varphi_{0}, \ldots, \varphi_{n-1}\right\}$, where $\varphi_{j}\left(a^{k}\right)=e^{\frac{2 \pi i 2 k}{n}}$ for $0 \leqslant j \leqslant n-1$ and $a^{k} \in H$.

2. The group of continuous characters on the circle group is the set $\widehat{\mathbb{T}}=\left\{\varphi_{k}: k \in \mathbb{Z}\right\}$, where $\varphi_{k}(x)=x^{k}$ for all $x \in \mathbb{T}([7], \S 4.1)$. As $\mathbb{T}$ is a compact group, $\widehat{\mathbb{T}}$ is a discrete group by Proposition 2.2.1.

3. The group of continuous characters on $\mathbb{R}$ is $\widehat{\mathbb{R}}=\left\{\varphi_{k}: k \in \mathbb{R}\right\}$, where $\varphi_{k}(x)=e^{2 \pi i k x}$ for all $x \in \mathbb{R}([7], \S 4.1)$.

Proposition 2.2.2. Let $H$ be a compact abelian group. Suppose $\varphi_{1}$ and $\varphi_{2}$ are two distinct characters in $\hat{H}$. Then $\left\langle\varphi_{1}, \varphi_{2}\right\rangle=0$.

Proof: Since $\varphi_{1}$ and $\varphi_{2}$ are distinct, there exists $z \in H$ such that $\varphi_{1}(z) \neq \varphi_{2}(z)$. Also, recall that $\overline{\varphi_{2}}=\varphi_{2}^{-1}$. Therefore we have that

$$
\begin{aligned}
\left\langle\varphi_{1}, \varphi_{2}\right\rangle & =\int_{H} \varphi_{1}(y) \overline{\varphi_{2}(y)} d y \\
& =\int_{H} \varphi_{1}(z y) \overline{\varphi_{2}(z y)} d y \\
& =\varphi_{1}(z) \overline{\varphi_{2}(z)} \int_{H} \varphi_{1}(y) \overline{\varphi_{2}(y)} d y \\
& =\varphi_{1}(z) \varphi_{2}^{-1}(z)\left\langle\varphi_{1}, \varphi_{2}\right\rangle
\end{aligned}
$$

As $\varphi_{1}(z) \varphi_{2}^{-1}(z) \neq 1$, we must have $\left\langle\varphi_{1}, \varphi_{2}\right\rangle=0$.

Proposition 2.2.2 shows that the continuous characters of a compact abelian group form an orthogonal set under the $L^{2}$ inner product. Furthermore, if the Haar measure $\mu_{H}$ on $H$ is normalized, then for $\varphi \in \hat{H}$ we have

$$
\langle\varphi, \varphi\rangle=\int_{H}|\varphi(y)|^{2} d y=\int_{H} 1 d y=\mu_{H}(H)=1
$$


as $\varphi(y) \in \mathbb{T}$ for all $y \in H$. Therefore, $\hat{H}$ is an orthonormal set in this case. We will now state an important theorem which will be used to prove Lemma 2.2.4. It is a particular version of the Gelfand-Raikov Theorem, which is proved in ([7] §3.3).

Theorem 2.2.3 (Gelfand-Raikov Theorem). Let $H$ be a LCA group. If $z \in H$ and $\varphi(z)=1$ holds for all $\varphi \in \hat{H}$, then $z=e$.

For a topological space $X$, let $C(X)$ be the set of continuous functions $g: X \rightarrow \mathbb{C}$, which is a $\mathbb{C}$-algebra under usual addition and multiplication of functions. When $X$ is a compact space, $C(X)$ is a normed space under the uniform norm $\|g\|_{\infty}=$ $\sup _{x \in X}|g(x)|$.

Lemma 2.2.4. Suppose $H$ is a compact abelzan group. Let $A=\operatorname{span}\{\hat{H}\}$, the set of fintte linear combinations over $\mathbb{C}$ of $\hat{H}$. Then $A$ is a dense subalgebra of $C(H)$ with respect to the uniform norm.

Proof: We will prove this by using the complex Stone-Weierstass Theorem ([17], $\S 5)$.

The set $A$ is clearly contained in $C(H)$. The product of two elements of $A$ is given by

$$
\left(\sum_{\imath=1}^{n} a_{\imath} \varphi_{\imath}\right)\left(\sum_{\jmath=1}^{m} b_{\jmath} \chi_{\jmath}\right)=\sum_{\imath=1}^{n} \sum_{\jmath=1}^{m} a_{\imath} b_{\jmath} \varphi_{\imath} \chi_{\jmath} \in A .
$$

Therefore $A$ is closed under multiplication. It is even easier to see that $A$ is also closed under addition, so that $A$ is a subalgebra of $C(H)$.

Suppose $g: H \rightarrow \mathbb{C}^{\times}$is a constant function given by $g(h)=c$ for all $h \in H$, where $c \in \mathbb{C}$. Then $g=c \varphi_{0} \in A$. Therefore the constant functions are in $A$. 
We prove that $A$ separates points. Take two elements $x \neq y$ in $H$, and suppose $\varphi(x)=\varphi(y)$ for every $\varphi \in \hat{H}$. Then for all $\varphi \in \hat{H}, \varphi\left(x y^{-1}\right)=1$. By the GelfandRaikov Theorem we have that $x y^{-1}=e$, or $x=y$, which is a contradiction.

$A$ is closed under complex conjugation, since

$$
\overline{\sum_{\imath=1}^{n} a_{\imath} \varphi_{\imath}}=\sum_{i=1}^{n} \overline{a_{\imath}} \varphi_{\imath}^{-1} \in A
$$

In summary $A$ is a subalgebra of $C(H)$ which contains the constant functions, separates points and is closed under complex conjugation. By the complex StoneWeierstrass Theorem, $A$ is dense in $C(H)$.

For a compact group $H$, let $[C(H)]$ be the set of equivalence classes $[g]$ in $L^{2}(H)$, with $g \in C(H)$, under the equivalence relation of $\mu_{H}$-almost everywhere equality. Similarly define $[\operatorname{span}\{\hat{H}\}]$ for a compact abelian group $H$. We will abuse notation a little bit by writing $C(H)$ and $\operatorname{span}\{\hat{H}\}$ when we are actually referring to the set of equivalence classes. In fact, in later chapters $C(H)$ will always be in reference to the subset of $L^{2}(H)$.

Suppose $X$ is a topological space. For a function $f: X \rightarrow \mathbb{C}$, define the support of $f$, denoted $\operatorname{supp}(f)$, as the closure of the set $\{x \in X: f(x) \neq 0\}$. The space of continuous functions of compact support, denoted by $C_{c}(X)$, is the set of all continuous functions $f: X \rightarrow \mathbb{C}$ where the support of $f$ is compact. The space $C_{c}(X)$ is a normed space with respect to the infinity norm, since the infinity norm of all compactly supported functions is finite. In ([10] 113$)$, it is proven that for a locally compact Hausdorff space $X$ with a non-zero Radon measure $\mu$, then the space $C_{c}(X)$ is dense in $L^{p}(X, \mu)$ for every $1 \leqslant p<\infty$, where of course $C_{c}(X)$ is being considered 
as a subset of $L^{p}(X, \mu)$. Recall from earlier that a Haar measure is a non-zero Radon measure. For a locally compact group $H$ with Haar measure $\mu_{H}$, the set $C_{c}(H)$ will refer to the set of equivalence classes of continuous and compactly supported functions on $H$ under the equivalence of $\mu_{H^{-}}$-almost everywhere equality. If $H$ is a compact group, then clearly $C_{c}(H)=C(H)$, so the following lemma is in fact a specific case of the previously mentioned theorem.

Lemma 2.2.5. Suppose $H$ is a compact group. Then $C(H)$ is dense in $L^{2}(H)$ with respect to the $L^{2}$-norm.

Suppose $H$ is a locally compact group with Haar measure $\mu_{H}$, and let $N=\left\{A \in \mathcal{B}(H): \quad \mu_{H}(A)=0\right\}$ be the null sets. Then $L^{\infty}\left(H, \mu_{H}\right)$ is the set of all equivalence classes of functions $g: H \rightarrow \mathbb{C}$ which satisfy that the infinity norm

$$
\|g\|_{\infty}=\inf _{\Delta \in N} \sup _{x \in \Delta^{c}}|g(x)|
$$

is finite. When $\mu_{H}$ is understood, we typically refer to the space as $L^{\infty}(H)$. It is a normed space under this infinity norm $([3], \S 3.3)$. When $H$ is compact, note that $C(H) \subseteq L^{\infty}(H)$ and this definition of the infinity norm will clearly coincide with our earlier definition of the uniform norm on $C(H)$. That means, for $g \in C(H)$, we have

$$
\sup _{x \in H}|g(x)|=\inf _{\Delta \in N} \sup _{x \in \Delta^{c}}|g(x)| .
$$

Lemma 2.2.6. Let $H$ be a compact group with a normalzzed Haar measure $\mu_{H}$. For $g \in L^{\infty}(H)$, we have that $\|g\|_{2} \leqslant\|g\|_{\infty}$. In particular, $L^{\infty}(H) \subseteq L^{2}(H)$. 
Proof: Suppose $g \in L^{\infty}(H)$. Then

$$
\begin{aligned}
\left(\|g\|_{2}\right)^{2} & =\int_{H}|g(x)|^{2} d x \\
& \leqslant \int_{H}\|g\|_{\infty}^{2} d x \\
& =\mu_{H}(H)\|g\|_{\infty}^{2} \\
& =\|g\|_{\infty}^{2}
\end{aligned}
$$

We have $\|g\|_{2} \leqslant\|g\|_{\infty}<\infty$. Therefore $g \in L^{2}(H)$, proving $L^{\infty}(H) \subseteq L^{2}(H)$.

Note that the assertion that $L^{\infty}(H) \subseteq L^{2}(H)$ holds for a compact group even if the Haar measure is not normalized. Normalization is only assumed to get $\|g\|_{2} \leqslant\|g\|_{\infty}$.

Lemma 2.2.7. Suppose $H$ is a compact abelian group (with normalized Haar measure). Then span $\{\hat{H}\}$ is dense in $C(H)$ with respect to the $L^{2}-$ norm.

Proof: Let $g \in C(H)$ and $\left(g_{n}\right)_{n=1}^{\infty}$ be a sequence in $\operatorname{span}\{\hat{H}\}$ which converges to $g$ with respect to the infinity norm. We can find such a sequence because, by Lemma 2.2.4, span $\{\hat{H}\}$ is dense in $C(H)$ with respect to the uniform norm, which coincides with the infinity norm. By Lemma 2.2.6, we have that

$$
\lim _{n \rightarrow \infty}\left\|g-g_{n}\right\|_{2} \leqslant \lim _{n \rightarrow \infty}\left\|g-g_{n}\right\|_{\infty}=0 .
$$

Therefore for every $g \in C(H)$, there exists a sequence $\left(g_{n}\right)_{n=1}^{\infty}$ in $\operatorname{span}\{\hat{H}\}$ which converges to $g$ with respect to the $L^{2}$-norm. This proves that $\operatorname{span}\{\hat{H}\}$ is dense in $C(H)$ with respect to the $L^{2}$-norm. 
When $H$ is compact and abelian, we have seen that $\operatorname{span}\{\hat{H}\}$ is dense in $C(H)$ and $C(H)$ is dense in $L^{2}(H)$, both with respect to the $L^{2}$-norm. In essence, the next proposition has already been proven.

Proposition 2.2.8. Suppose $H$ is a compact abelian group (with normalized Haar measure). Then span $\{\hat{H}\}$ is dense in $L^{2}(H)$ with respect to the $L^{2}{ }^{-}$norm.

Proof: Consider the closure operation in $L^{2}(H)$ with respect to the $L^{2}$-norm. Lemma 2.2.7 shows that $C(H) \subseteq \overline{\operatorname{span}\{\hat{H}\}}$, thus $\overline{C(H)} \subseteq \overline{\operatorname{span}\{\hat{H}\}}$. Lemma 2.2.5 shows that $L^{2}(H) \subseteq \overline{C(H)}$, and therefore $L^{2}(H) \subseteq \overline{\operatorname{span}\{\hat{H}\}}$. This implies that $L^{2}(H)=$ $\overline{\operatorname{span}\{\hat{H}\}}$, proving the density of $\operatorname{span}\{\hat{H}\}$ in $L^{2}(H)$.

To summarize, Proposition 2.2.2 shows that the continuous characters on a compact abelian group $H$ are an orthogonal set in $L^{2}(H)$. We remarked that this is an orthonormal set when the Haar measure on $H$ is normalized. Proposition 2.2.8 shows that, for a compact abelian group $H$ with normalized Haar measure, the closure of the linear span of $\hat{H}$ in $L^{2}(H)$ is all of $L^{2}(H)$. We have therefore shown that for a compact abelian group $H$ with normalized Haar measure, the continuous characters form an orthonormal Hilbert space basis for $L^{2}(H)$.

It follows from a more general theorem in ([8], Thm 24.14) that $H$ is second countable if and only if $L^{2}(H)$ has a countable orthonormal basis. Thus, if we further suppose that $H$ is second countable, then our orthonormal Hilbert space basis is countable. We can enumerate it as $\hat{H}=\left\{\varphi_{1}, \varphi_{2}, \ldots\right\}$. It follows that for every $g \in L^{2}(H)$, we have

$$
g=\sum_{\varphi \in \hat{H}}\langle g, \varphi\rangle \varphi
$$

Equivalently, this means that $\lim _{n \rightarrow \infty}\left\|g-\sum_{i=1}^{n}\left\langle g, \varphi_{\imath}\right\rangle \varphi_{\imath}\right\|_{2}=0$. 


\section{Chapter 3}

\section{The Poisson Summation Formula}

In this chapter, we will fix a LCA group $G$ with a discrete subgroup $\Gamma$ such that the quotient group $G / \Gamma$ is compact. For a function $f \in C_{c}(G)$, we will define the $\Gamma-$ projection $f_{\Gamma} \in C(G / \Gamma)$. We will also define a measure $\mu_{G}$ on $G$ and use it to define a measure $\mu_{G / \Gamma}$ on $G / \Gamma$, called the quotient measure. These measures are related in such a way that integrating a function $f \in C_{c}(G)$ over $G$ is the same as integrating $f_{\Gamma}$ over $G / \Gamma$. We will define the Fourier transform and the Fourier inverse, and use Fourier inversion to prove the main result of the chapter, the Poisson Summation Formula.

\subsection{Invariant Measure On The Quotient Space}

Let $G$ be a second countable LCA group with a discrete subgroup $\Gamma$ such that the group $G / \Gamma$, equipped with the quotient topology induced by the quotient map $\pi: G \rightarrow$ $G / \Gamma$, is compact. We will denote the identity of $G$ as $e . G / \Gamma$ is a topological group, as mentioned in $\S 2.1$, and we shall begin by proving that $G / \Gamma$ is a second countable LCA group. We will then define the $\Gamma$-projection $f_{\Gamma}$ for a function $f \in C_{c}(G)$, as well 
as two (left) Haar measures $\mu_{G}$ and $\mu_{G / \Gamma}$ on $G$ and $G / \Gamma$, respectively. The measure $\mu_{G / \Gamma}$ will be defined in such a way that it satisfies

$$
\int_{G} f(x) d x=\int_{G / \Gamma} f_{\Gamma}(x \Gamma) d(x \Gamma)
$$

for every $f \in C_{c}(G)$.

Lemma 3.1.1. $\Gamma$ is a closed set in $G$.

Proof: It suffices to show that the complement $\Gamma^{c}$ is open. Let $U_{1}$ be open in $G$ such that $e \in U_{1}$ and $U_{1} \cap \Gamma=\{e\}$. Such an open set $U_{1}$ can be found since $\Gamma$ is discrete. Let $U$ be a symmetric open neighbourhood of $e$ contained in $U_{1}$, as mentioned in $\S 2.1$. Then $U \cap \Gamma=\{e\}$, and for any $\gamma \in \Gamma, \gamma U \cap \Gamma=\{\gamma\}$.

Suppose $\Gamma^{c}$ is not open. Then there exists $x \in \Gamma^{c}$ such that for every open neighbourhood $B$ of $e, x B \cap \Gamma \neq \varnothing$. In particular, there exists $\gamma \in x U \cap \Gamma \neq \varnothing$, and thus $\gamma=x u$ for some $u \in U$. If $\gamma_{1} \in x U \cap \Gamma$, then $\gamma_{1}=x u_{1}$ for some $u_{1} \in U$. We look to show that $\gamma_{1}=\gamma$. Since $\Gamma$ is a subgroup, $\gamma \gamma_{1}^{-1} \in \Gamma$. But $\gamma^{-1} \gamma_{1}=u_{1}^{-1} x^{-1} x u=u_{1}^{-1} u \in U$. Thus $\gamma \gamma_{1}^{-1} \in U \cap \Gamma=\{e\}$, implying $\gamma=\gamma_{1}$. Therefore, we have shown that $x U \cap \Gamma=\{\gamma\}$.

Since $\mathrm{G}$ is Hausdorff, there is an open subset $V$ of $G$ such that $e \in V$ and $x^{-1} \gamma \notin V$, or equivalently $\gamma \notin x V$. Since $U \cap V$ is an open neighbourhood of $e,(x(U \cap V)) \cap \Gamma \neq \varnothing$. Because $(x(U \cap V)) \cap \Gamma \subseteq x U \cap \Gamma=\{\gamma\}$, we have $\gamma \in(x(U \cap V)) \cap \Gamma$. However $x(U \cap V) \subseteq x V$ and $\gamma \notin x V$ by construction, implying that $\gamma \notin x(U \cap V)$. This gives a contradiction.

Therefore $\Gamma^{c}$ must be open, proving that $\Gamma$ is closed. 
Note that the proof of Lemma 3.1.1 can be applied to a more general situation to show that discrete subgroups of a LCA group are closed.

Proposition 3.1.2. $G / \Gamma$ is a second countable $L C A$ group.

Proof: $\quad G / \Gamma$ is clearly abelian. The identity $e \Gamma$ of $G / \Gamma$ has the compact neighbourhood $G / \Gamma$, thus $G / \Gamma$ is locally compact ( $\{2.1)$. Second countability follows easily from $G$ being second countable. Only the Hausdorff property is left to be shown. By a remark made in $\S 2.1$, it suffices to show that the identity set $\{e \Gamma\}$ is closed in $G / \Gamma$.

By definition, $F \subseteq G / \Gamma$ is closed in the quotient topology if and only if $\pi^{-1}(F)$ is closed in $G([23] \S 3.4)$. By Lemma 3.1.1 we have that $\Gamma=\pi^{-1}(\{e \Gamma\})$ is closed. Therefore $\{e \Gamma\}$ is closed in $G / \Gamma$.

Lemma 3.1.3. Let $H$ be a topological group. If $K$ is a compact subset of $H$ and $D$ is a discrete subgroup of $H$, then $K \cap D$ is finite.

Proof: Since $K \cap D \subseteq D$, it is clear that $K \cap D$ is discrete. The subgroup $D$ is closed by an earlier remark, and $K$ is defined to be closed. Thus $K \cap D$ is a closed subset of a compact subset $K$ of $H$, showing that $K \cap D$ is also a compact subset of $H$. Since $K \cap D$ is discrete, $\bigcup_{x \in K \cap D}\{x\}$ is an open cover of the compact space $K \cap D$, and must therefore have a finite subcover. But the only possible subcover is $\bigcup_{x \in K \cap D}\{x\}$, proving that $K \cap D$ must be finite.

A cross section is a map $s: G / \Gamma \rightarrow G$ which satisfies $\pi(s(y))=y$ for every $y \in G / \Gamma$. Essentially, a cross section assigns a coset representative for each element of $G / \Gamma$. The 
existence of a cross section for the quotient map $\pi: G \rightarrow G / \Gamma$ whose image is a Borel set of $G$ is mentioned in ([7], §2.7). We fix such a cross section $s: G / \Gamma \rightarrow G$. From now on, unless mentioned otherwise, every $y \in G / \Gamma$ will be denoted by $x \Gamma$, where $x=s(y)$. In other words, we denote elements of the coset space with a representative given by the cross section. This is to avoid confusion when we want to use a coset representative of an element in $G / \Gamma$, so that we will know which representative we are referring to.

For $f \in C_{c}(G)$, we define the $\Gamma$-projection of $f, f_{\Gamma}: G / \Gamma \rightarrow \mathbb{C}$, by

$$
f_{\Gamma}(x \Gamma)=\sum_{\gamma \in \Gamma} f(x \gamma)
$$

for every $x \Gamma \in G / \Gamma$. Note that, as mentioned above, $x$ is given by our fixed cross section, making this definition well-defined. It is worth noting that this definition is well-defined even if we take a general element $y \Gamma \in G / \Gamma$ where $y$ is not given by the cross section. Lemma 3.1.3 assures us that the sum on the right-hand side in (3.1) is finite, and therefore always makes sense. The $\Gamma$-projection is an important function that will be seen many times throughout, including the main result of this chapter, the Poisson Summation Formula.

Theorem 3.1.4. For $f \in C_{c}(G), f_{\Gamma}$ is continuous.

Proof: This will be proved by showing that the convergence of nets is preserved under $f_{\Gamma}$. For those unfamiliar with nets in a topological space, see ([23], §4.2).

Let $\left(x_{\lambda}\right)_{\lambda \in \Lambda}$ be a net in $G$ which converges to some $c \in G$. It suffices to show that $\left(f_{\Gamma}\left(x_{\lambda} \Gamma\right)\right)_{\lambda \in \Lambda}$ converges to $f_{\Gamma}(c \Gamma)$. 
Let $V_{1}$ be a compact neighbourhood of $e$. Set $V=V_{1} \cap V_{1}^{-1}$, so that $V$ is a compact symmetric neighbourhood of $e$. For $\gamma \in \Gamma$, we have

$$
\begin{aligned}
c \gamma V \cap \operatorname{supp}(f) \neq \varnothing & \Leftrightarrow \gamma V \cap c^{-1} \operatorname{supp}(f) \neq \varnothing \\
& \Leftrightarrow \gamma \in c^{-1} \operatorname{supp}(f) V
\end{aligned}
$$

Both $\operatorname{supp}(f)$ and $V$ are compact. Since $\operatorname{supp}(f) V$ is the continuous image of the compact set $\operatorname{supp}(f) \times V$ under multiplication, the product $\operatorname{supp}(f) V$ is compact. Therefore $c^{-1} \operatorname{supp}(f) V$ is a compact set, being the homeomorphic image of $\operatorname{supp}(f) V$ under the left translation $x \mapsto c^{-1} x$.

The set $\Gamma \cap c^{-1} \operatorname{supp}(f) V$ is the intersection of a discrete and compact set, so by Lemma 3.1.3 it is finite. So there are only finitely many $\gamma \in \Gamma$ such that $c \gamma V \cap$ $\operatorname{supp}(f) \neq \varnothing$. Denote the finite set of $\gamma \in \Gamma$ such that $c V \gamma \cap \operatorname{supp}(f) \neq \varnothing$ by $\left\{\gamma_{1}, \ldots, \gamma_{n}\right\}$

Consider the compact neighbourhood $c V$ of $c$. Since $\left(x_{\lambda}\right)_{\lambda \in \Lambda}$ converges to $c,\left(x_{\lambda}\right)_{\lambda \in \Lambda}$ will be residually in $c V$. That is there exists $\lambda_{V} \in \Lambda$ such that when $\lambda \geqslant \lambda_{V}, x_{\lambda} \in c V$.

The collection of sets of the form $D(z, R)$, the open disc in $\mathbb{C}$ of radius $R$ centered at $z \in \mathbb{C}$, forms a neighbourhood base of $z$ in $\mathbb{C}$. Let $U=D\left(f_{\Gamma}(c \Gamma), R\right)$, where $R>0$. We are looking to show that the net $\left(f_{\Gamma}\left(x_{\lambda} \Gamma\right)\right)_{\lambda \in \Lambda}$ is residually in $U$. For each $j$ with $1 \leqslant j \leqslant n$, the net $\left(x_{\lambda} \gamma_{\jmath}\right)_{\lambda \in \Lambda}$ converges to $c \gamma_{\jmath}$. Since $f$ is continuous, for each $j$ the net $\left(f\left(x_{\lambda} \gamma_{\jmath}\right)\right)_{\lambda \in \Lambda}$ is residually in $D\left(f\left(c \gamma_{\jmath}\right), R / n\right)$, so there exists $\lambda_{\jmath}$ such that when $\lambda \geqslant \lambda_{\jmath}, f\left(x_{\lambda} \gamma_{\jmath}\right) \in D\left(f\left(c \gamma_{\jmath}, R / n\right)\right.$. Let $\lambda_{U} \geqslant \lambda_{V}, \lambda_{1}, \ldots, \lambda_{n}$ and $\lambda \geqslant \lambda_{U}$. Since $\lambda \geqslant \lambda_{V}$, $x_{\lambda} \in c V$ and thus

$$
f_{\Gamma}\left(x_{\lambda} \Gamma\right)=\sum_{\gamma \in \Gamma} f\left(x_{\lambda} \gamma\right)=\sum_{\jmath=1}^{n} f\left(x_{\lambda} \gamma_{\jmath}\right)
$$


Since $f\left(x_{\lambda} \gamma_{\jmath}\right) \in D\left(f\left(c \gamma_{\jmath}\right), R / n\right)$ for every $\jmath$, we see that

$$
\begin{aligned}
\left|f_{\Gamma}\left(x_{\lambda} \Gamma\right)-f_{\Gamma}(c \Gamma)\right| & =\left|\sum_{\jmath=1}^{n}\left(f\left(x_{\lambda} \gamma_{\jmath}\right)-f\left(c \gamma_{\jmath}\right)\right)\right| \\
& \leqslant \sum_{\jmath=1}^{n}\left|f\left(x_{\lambda} \gamma_{\jmath}\right)-f\left(c \gamma_{\jmath}\right)\right| \\
& \leqslant \sum_{\jmath=1}^{n} R / n \\
& =R .
\end{aligned}
$$

Therefore, $f_{\Gamma}\left(x_{\lambda} \Gamma\right) \in U$, meaning the net $\left(f_{\Gamma}\left(x_{\lambda} \Gamma\right)\right)_{\lambda \in \Lambda}$ is residually in every open neighbourhood of $f_{\Gamma}(c \Gamma)$, proving that $\left(f_{\Gamma}\left(x_{\lambda} \Gamma\right)\right)_{\lambda \in \Lambda}$ converges to $f_{\Gamma}(c \Gamma)$. This has shown that $f_{\Gamma}$ is continuous.

It is proven in $([7], \S 2.6)$ that the map $P: C_{c}(G) \rightarrow C(G / \Gamma)$ given by the $\Gamma$-projection, $P(f)=f_{\Gamma}$ for all $f \in C_{c}(G)$, is surjective. Thus every continuous function on $G / \Gamma$ can be written as the $\Gamma$-projection of some $f \in C_{c}(G)$.

We now define two (left) Haar measures, $\mu_{G}$ and $\mu_{G / \Gamma}$, on $G$ and $G / \Gamma$, respectively. The measure $\mu_{G / \Gamma}$ will be known as the quotzent measure on $G / \Gamma$ with respect to $\left(G, \mu_{G}\right)$, or simply as the quotient measure. We begin by fixing a (left) Haar measure $\mu$ on $G$. The proceeding lemma follows from ([7], Thm 2.49, Cor 2.51).

Lemma 3.1.5. There exists a (left) Haar measure $\nu$ of $G / \Gamma$ such that, for $f \in C_{c}(G)$, we have

$$
\int_{G} f(x) d \mu(x)=\int_{G / \Gamma} f_{\Gamma}(x \Gamma) d \nu(x \Gamma)=\int_{G / \Gamma} \sum_{\gamma \in \Gamma} f(x \gamma) d \nu(x \Gamma)
$$

The measure $\nu$ is finite, as $G / \Gamma$ is compact $(\S 2.1)$. We set $\mu_{G}=\left(\frac{1}{\nu(G / \Gamma)}\right) \mu$ and 
$\mu_{G / \Gamma}=\left(\frac{1}{\nu(G / \Gamma)}\right) \nu$. We do so to normalize the measure $\mu_{G / \Gamma}$. This gives us the following desired property.

Theorem 3.1.6. If $f \in C_{c}(G)$, then we have

$$
\int_{G} f(x) d \mu_{G}(x)=\int_{G / \Gamma} f_{\Gamma}(x \Gamma) d \mu_{G / \Gamma}(x \Gamma)=\int_{G / \Gamma} \sum_{\gamma \in \Gamma} f(x \gamma) d \mu_{G / \Gamma}(x \Gamma) .
$$

Proof: For $f \in C_{c}(G)$, we have

$$
\begin{aligned}
\int_{G} f(x) d \mu_{G}(x) & =\frac{1}{\nu(G / \Gamma)} \int_{G} f(x) d \mu(x) \\
& =\frac{1}{\nu(G / \Gamma)} \int_{G / \Gamma} f_{\Gamma}(x \Gamma) d \nu(x \Gamma) \quad \text { by Lemma 3.1.5 } \\
& =\int_{G / \Gamma} f_{\Gamma}(x \Gamma) d \mu_{G / \Gamma}(x \Gamma) \\
& =\int_{G / \Gamma} \sum_{\gamma \in \Gamma} f(x \gamma) d \mu_{G / \Gamma}(x \Gamma) .
\end{aligned}
$$

It is mentioned in $([7], \S 2.7)$ that Theorem 3.1.6 holds true for every function $f \in L^{1}(G)$. Therefore, we can and will use Theorem 3.1.6 even for functions that may not be in $C_{c}(G)$, just as long as they are in $L^{1}(G)$. Of course in this case we would not speak about the $\Gamma$-projection, since we only defined it for functions in $C_{c}(G)$. Instead, we just get that $\int_{G} f(x) d \mu_{G}(x)=\int_{G / \Gamma} \sum_{\gamma \in \Gamma} f(x \gamma) d \mu_{G / \Gamma}(x \Gamma)$ for $f \in L^{1}(G)$.

Unless specified otherwise, from now on when we integrate on $G$ it is with respect to the Haar measure $\mu_{G}$, and when we integrate on $G / \Gamma$ it is with respect to the Haar measure $\mu_{G / \Gamma}$. 


\subsection{The Poisson Summation Formula}

In this section, we will define the Fourier transform for a LCA group and present proofs of both the Fourier Inversion Theorem and Poisson Summation Formula for a compact abelian group.

Let $H$ be a LCA group with (left) Haar measure $\mu_{H}$ and $g \in L^{1}(H)$. Define the Fourler transform of $g$, denoted $\widehat{g}: \widehat{H} \rightarrow \mathbb{C}$, by

$$
\hat{g}(\varphi)=\int_{H} g(x) \overline{\varphi(x)} d x
$$

where the integral is taken with respect to the Haar measure $\mu_{H}$. For every $\varphi \in \hat{H}$, we have that $\bar{\varphi}=\varphi^{-1} \in L^{\infty}(H)$, which means that $g \bar{\varphi} \in L^{1}(H)([3], \S 3.3)$ and thus is integrable.

Now suppose that $H$ is a compact abelian group and that $g \in l^{1}\left(\hat{H}, \mu_{\hat{H}}\right)$, where $\mu_{\widehat{H}}$ is the counting measure on $\widehat{H}$. Define the inverse Fourzer transform $\breve{g}: H \rightarrow \mathbb{C}$ by

$$
\check{g}(x)=\sum_{\varphi \in \hat{H}} g(\varphi) \varphi(x) .
$$

Note that by definition this sum converges absolutely, following from the fact that $g \in l^{1}(\hat{H})$.

The Fourier transform and inverse Fourier transform are related to each other by the well-known Fourner Inversion Theorem.

Theorem 3.2.1. Fourner Inversion Theorem: Let $H$ be a second countable compact abelian group and suppose $g \in C(H)$ is such that $\hat{g} \in l^{1}(\hat{H})$. Then $g=\check{\widehat{g}}$. 
Proof: Recall the $L^{2}$ inner product on $H(\S 2.1)$ given by $\left\langle g_{1}, g_{2}\right\rangle=\int_{H} g_{1}(x) \overline{g_{2}(x)} d x$, where integration is taken with respect to a (left) Haar measure on $H$. We may also assume that this measure on $H$ is normalized. By Proposition 2.2.2, Proposition 2.2.8, and the assumption of second countability, $\hat{H}$ is a countable Hilbert basis for $L^{2}(H)$. As $g \in C(H) \subseteq L^{2}(H)$, we can write

$$
\begin{array}{rlr}
g & =\sum_{\varphi \in \hat{H}}\langle g, \varphi\rangle \varphi & \\
& =\sum_{\varphi \in \hat{H}}\left(\int_{H} g(y) \overline{\varphi(y)} d y\right) \varphi & \text { by }(2.1) \\
& =\sum_{\varphi \in \hat{H}} \hat{g}(\varphi) \varphi . & \text { by }(3.2)
\end{array}
$$

That $\sum_{\varphi \in \hat{H}} \hat{g}(\varphi) \varphi$ converges uniformly to a continuous function follows from ([13], Thm 2.2 and Thm 2.3) which requires our assumption that $\hat{H}$ be countable. Let $h$ denote this continuous function. Note that $\sum_{\varphi \in \hat{H}} \hat{g}(\varphi) \varphi$ converges to $h$ in the $L^{\infty}-$ norm. Since $\|h\|_{2} \leqslant\|h\|_{\infty}$ for all $h \in L^{2}(H)$ (Lemma 2.2.6), we have that $\sum_{\varphi \in \hat{H}} \widehat{g}(\varphi) \varphi$ converges to $h$ in the $L^{2}$-norm. We know that $\sum_{\varphi \in \hat{H}} \hat{g}(\varphi) \varphi$ converges to $g$ in the $L^{2}$-norm. It follows that $h=g$ almost everywhere. Since $h-g$ is a continuous function and our measure on $H$ is non-zero on open sets (by Lemma 2.1.1), $h-g=0$ everywhere.

As $\sum_{\varphi \in \hat{H}} \hat{g}(\varphi) \varphi$ converges uniformly to $h=g$, it also converges pointwise to $g$, meaning that

$$
g(x)=\sum_{\varphi \in \hat{H}} \hat{g}(\varphi) \varphi(x)=\check{\widehat{g}}(x)
$$

for every $x \in H$. 
For $\varphi \in \widehat{G / \Gamma}$, define $\varphi^{\prime}: G \rightarrow \mathbb{T}$ by

$$
\varphi^{\prime}(x)=\varphi(x \Gamma)
$$

We show that $\varphi^{\prime} \in \widehat{G}$. It is continuous, as $\varphi^{\prime}$ is the composition of the continuous functions $\varphi$ and $\pi$, where $\pi$ is the quotient map $\pi(x)=x \Gamma$. It preserves the group law, since for $x, y \in G$

$$
\varphi^{\prime}(x y)=\varphi(x y \Gamma)=\varphi(x \Gamma) \varphi(y \Gamma)=\varphi^{\prime}(x) \varphi^{\prime}(y)
$$

Lemma 3.2.2. For $f \in C_{c}(G)$ and $\varphi \in \widehat{G / \Gamma}$, we have $\widehat{f}_{\Gamma}(\varphi)=\widehat{f}\left(\varphi^{\prime}\right)$.

Proof: Let $f_{\varphi}: G \rightarrow \mathbb{C}$ be defined by

$$
f_{\varphi}(y)=f(y) \overline{\varphi^{\prime}(y)}
$$

for all $y \in G$. Note that $f_{\varphi} \in C_{c}(G)$. We have that

$$
\begin{aligned}
\hat{f}_{\Gamma}(\varphi) & =\int_{G / \Gamma} f_{\Gamma}(y \Gamma \overline{\varphi(y \Gamma)} d(y \Gamma) \\
& =\int_{G / \Gamma} \sum_{\gamma \in \Gamma} f(y \gamma) \overline{\varphi^{\prime}(y \gamma)} d(y \Gamma) \\
& =\int_{G / \Gamma} \sum_{\gamma \in \Gamma} f_{\varphi}(y \gamma) d(y \Gamma) \\
& =\int_{G} f_{\varphi}(y) d y \\
& =\int_{G} f(y) \overline{\varphi^{\prime}(y)} d y \\
& =\hat{f}\left(\varphi^{\prime}\right) .
\end{aligned}
$$


We can finally state the main theorem we have been working towards in this chapter.

Theorem 3.2.3 (Poisson Summation Formula). For $f \in C_{c}(G)$ such that $\hat{f}_{\Gamma} \in l^{1}(\widehat{G / \Gamma})$, we have that

$$
f_{\Gamma}(x \Gamma)=\sum_{\gamma \in \Gamma} f(x \gamma)=\sum_{\varphi \in \widehat{G / \Gamma}} \hat{f}\left(\varphi^{\prime}\right) \varphi^{\prime}(x)
$$

The sum on the right-hand side converges absolutely. In particular

$$
f_{\Gamma}(e \Gamma)=\sum_{\gamma \in \Gamma} f(\gamma)=\sum_{\varphi \in \widehat{G / \Gamma}} \hat{f}\left(\varphi^{\prime}\right)
$$

Proof: By the Fourier Inversion Theorem, which holds true since $\widehat{f}_{\Gamma} \in l^{1}(\widehat{G / \Gamma})$, we have

$$
\begin{aligned}
f_{\Gamma}(x \Gamma) & =\sum_{\varphi \in \widehat{G / \Gamma}} \hat{f}_{\Gamma}(\varphi) \varphi(x \Gamma) \\
& =\sum_{\varphi \in \widehat{G / \Gamma}} \hat{f}\left(\varphi^{\prime}\right) \varphi(x \Gamma) \quad \text { by Lemma 3.2.2 } \\
& =\sum_{\varphi \in \widehat{G / \Gamma}} \hat{f}\left(\varphi^{\prime}\right) \varphi^{\prime}(x) .
\end{aligned}
$$

Example: Let $G=\mathbb{R}$ and $\Gamma=\mathbb{Z}$. The group of irreducible characters on $\mathbb{R} / \mathbb{Z}$ is the set $\widehat{\mathbb{R} / \mathbb{Z}}=\left\{\varphi_{k}: k \in \mathbb{Z}\right\}$, where $\varphi_{k}(x+\mathbb{Z})=e^{2 \pi i k x}$ for all $x+\mathbb{Z} \in \mathbb{R} / \mathbb{Z}$. Recall that $\widehat{\mathbb{R} / \mathbb{Z}}$ is a discrete group by Proposition 2.2.1, and clearly isomorphic to $\mathbb{Z}$. Since the set $\left\{\varphi^{\prime}: \varphi \in \widehat{\mathbb{R} / \mathbb{Z}}\right\}$ is a subgroup of $\widehat{\mathbb{R}}$ isomorphic to $\mathbb{Z}$, we can consider the restriction of the Fourier transform of a function $f \in L^{1}(\mathbb{R})$ to this subgroup as a function on the integers, meaning $\hat{f}: \mathbb{Z} \rightarrow \mathbb{C}$. For a function $f \in C_{c}(\mathbb{R})$, we have the classical 
Poisson Summation Formula given by

$$
\sum_{n \in \mathbb{Z}} f(n)=\sum_{k \in \mathbb{Z}} \hat{f}(k) .
$$

whenever the right-hand side converges absolutely. 


\section{Chapter 4}

\section{Traces of Linear Operators on}

$L^{2}(G / \Gamma)$

We continue to work with the assumptions from Chapter 3 that $G$ is a second countable LCA group with a discrete subgroup $\Gamma$ such that the quotient group $G / \Gamma$ is compact. For a finite topological group $H$ equipped with the discrete topology, the (right) regular representation $R: H \rightarrow \operatorname{End}(\mathbb{C}[H])$ is defined by $(R(x) g)(y)=g(y x)$, where $x, y \in H$ and $g \in \mathbb{C}[H]$. Here $\mathbb{C}[H]$ denotes the space of functions $f: H \rightarrow \mathbb{C}$. In this chapter, we will extend this idea by defining the (right) regular representation $\bar{R}$ on the compact group $G / \Gamma$, and provide a construction of a related representation $R$ of $G$. For $f \in C_{c}(G)$, we use these representations to define associated bounded linear operators $R_{f}$ and $\bar{R}_{f_{\Gamma}}$ on $L^{2}(G / \Gamma)$. Our goal is to determine the trace of both of these operators and show how they relate to one another. We will see that the traces of both are the same, and equal to terms in the Poisson Summation Formula applied to $f$. 


\subsection{The Regular Representation of $G / \Gamma$}

We will introduce the representation $\bar{R}$ of $G / \Gamma$, as well as a linear operator $\bar{R}_{f}$ on $L^{2}(G / \Gamma)$, where $f \in C_{c}(G)$. We will compute the trace of $\bar{R}_{f}$ and provide an example.

For a non-zero Hilbert space $\mathcal{H}$, the set $\mathcal{L}(\mathcal{H})$ is the space of bounded linear operators on $\mathcal{H}$, which is an algebra under usual addition and composition of linear operators. Let $\operatorname{Aut}(\mathcal{H})$ denote the unit group of $\mathcal{L}(\mathcal{H})$, which forms a group under the group law of composition. Let $\mathcal{U}(\mathcal{H})$ denote the subgroup of $\operatorname{Aut}(\mathcal{H})$ consisting of unitary operators on $\mathcal{H}$. A representation $\rho$ of a locally compact topological group $H$ is a group homomorphism $\rho: H \rightarrow \operatorname{Aut}(\mathcal{H})$ which is continuous with respect to the strong operator topology on $\operatorname{Aut}(\mathcal{H})([7]$, Appendix 2), where $\mathcal{H}$ is a non-zero complex Hilbert space. If we replace $\operatorname{Aut}(\mathcal{H})$ with $\mathcal{U}(\mathcal{H})$, then we call $\rho$ a unitary representation of $H$. We will typically only consider representations which are unitary. It is proven in ([8], Cor 22.14) that every representation of a compact group is equivalent to a unitary representation.

To show continuity in the strong operator topology, it suffices to prove that for $v \in \mathcal{H}$ and for a convergent net $h \rightarrow e$ in $H$, we have $\rho(h) v \rightarrow v$ in $\mathcal{H}$, as mentioned in ([7], $\S 3.1)$. Note that any finite-dimensional non-zero complex vector space $V$ is a Hilbert space with respect to some inner product on $V([6], \S 1.12)$. It doesn't matter which inner product we choose, because all norms on a finite-dimensional vector space are equivalent ([18], Prop 4.1.3). In this case $\operatorname{Aut}(V)$ is the group $G L(V)$ of invertible linear transformations of $V$, and $\mathcal{U}(V)$ is the group of unitary linear transformations. 
Let $\bar{R}: G / \Gamma \rightarrow \mathcal{U}\left(L^{2}(G / \Gamma)\right)$ be defined by

$$
(\bar{R}(x) g)(y)=\left(\bar{R}_{x} g\right)(y)=g(y x)
$$

for all $x, y \in G / \Gamma$ and all $g \in L^{2}(G / \Gamma)$.

Proposition 4.1.1. $\bar{R}$ is a unitary representation of $G / \Gamma$.

Proof: Let $x \in G / \Gamma$. First we prove that in fact $\bar{R}_{x}$ is indeed a unitary operator. Note that $\bar{R}_{x}$ being invertible will follow from the homomorphic property, as $\bar{R}_{x} \bar{R}_{x^{-1}}=\bar{R}_{e \Gamma}$, where clearly $\bar{R}_{e \Gamma}$ is the identity of $\mathcal{U}\left(L^{2}(G / \Gamma)\right)$. It is clear that $\bar{R}_{x}$ is linear. Observe that

$$
\begin{aligned}
\left\|\bar{R}_{x} g\right\|_{2} & =\sqrt{\int_{G / \Gamma}|g(y x)|^{2} d(y)} \\
& =\sqrt{\int_{G / \Gamma}|g(y)|^{2} d(y)} \\
& =\|g\|_{2}
\end{aligned}
$$

for every $g \in L^{2}(G / \Gamma)$. Thus, $\bar{R}_{x}$ is a unitary operator.

To show that $\bar{R}$ is a representation of $G / \Gamma$, we must prove it is a continuous homomorphism. To show $\bar{R}$ is continuous, it suffices to show that as $x \rightarrow e \Gamma$ in $G / \Gamma$ and for $g \in L^{2}(G / \Gamma)$, we have $\bar{R}_{x} g \rightarrow g$ with respect to the $L^{2}$-norm. Let $\epsilon>0$, and fix $g \in L^{2}(G / \Gamma)$. Lemma 2.2.5 showed that continuous functions are dense in $L^{2}(G / \Gamma)$, so let $h \in C(G / \Gamma)$ be such that

$$
\|h-g\|_{2}<\frac{\epsilon}{3}
$$

As $h$ is continuous, there exists a neighbourhood $U$ of $e \Gamma$ such that for every $x$ in the 
net that are eventually in $U$ we have

$$
|h(y x)-h(y)|<\frac{\epsilon}{3}
$$

We proved earlier that $\left\|\bar{R}_{x} g-\bar{R}_{x} h\right\|_{2}=\|g-h\|_{2}$. Thus, we have that

$$
\begin{aligned}
\left\|\bar{R}_{x} g-g\right\|_{2} & \leqslant\left\|\bar{R}_{x} g-\bar{R}_{x} h\right\|_{2}+\left\|\bar{R}_{x} h-h\right\|_{2}+\|h-g\|_{2} \\
& =\|g-h\|_{2}+\sqrt{\int_{G / \Gamma}|h(y x)-h(y)|^{2} d y}+\|h-g\|_{2} \\
& <\frac{\epsilon}{3}+\frac{\epsilon}{3}+\frac{\epsilon}{3} \\
& =\epsilon .
\end{aligned}
$$

Therefore, $\left\|\bar{R}_{x} g-g\right\|_{2}<\epsilon$, proving that $\bar{R}_{x} g \rightarrow g$ with respect to the $L^{2}-$ norm for every $g \in L^{2}(G / \Gamma)$.

All that remains to be proven is that $\bar{R}$ preserves the group law of $G / \Gamma$. Suppose $x, y \in G / \Gamma$. We need to show that $\bar{R}_{x y}=\bar{R}_{x} \bar{R}_{y}$. Suppose that $g \in L^{2}(G / \Gamma)$ and $z \in G / \Gamma$. Then

$$
\left(\bar{R}_{x}\left(\bar{R}_{y} g\right)\right)(z)=\left(\bar{R}_{y} g\right)(z x)=g(z x y)=\bar{R}_{x y} g(z)
$$

Thus $\bar{R}_{x y} g=\bar{R}_{x} \bar{R}_{y} g$ for every $g \in L^{2}(G / \Gamma)$, proving that $\bar{R}_{x y}=\bar{R}_{x} \bar{R}_{y}$.

It is clear that $\bar{R}$ is well-defined. We call $\bar{R}$ the (right) regular representation of $G / \Gamma$. 


\section{Example:}

Consider $G=\mathbb{R}$ and $\Gamma=\mathbb{Z}$. Let $x+\mathbb{Z}, z+\mathbb{Z} \in \mathbb{R} / \mathbb{Z}$ and $g \in L^{2}(\mathbb{R} / \mathbb{Z})$. The regular representation $\bar{R}$ of $\mathbb{R} / \mathbb{Z}$ is defined by $(\bar{R}(x+\mathbb{Z}) g)(z+\mathbb{Z})=g(z+x+\mathbb{Z})$. Visually, $\bar{R}(x+\mathbb{Z})$ shifts the function $g$ on $\mathbb{R} / \mathbb{Z} \cong \mathbb{T}$ clockwise by $x$.

Let $f \in C(G / \Gamma)$. Note that the space $C(G / \Gamma)$ is being considered as a subspace of $L^{2}(G / \Gamma)$, and is thus the space of all functions in $L^{2}(G / \Gamma)$ that are equal $\mu_{G / \Gamma \text {-almost }}$ everywhere to a continuous function. Define $\bar{R}_{f}: L^{2}(G / \Gamma) \rightarrow L^{2}(G / \Gamma)$ by

$$
\left(\bar{R}_{f} g\right)(y)=\int_{G / \Gamma} f(x)\left(\bar{R}_{x} g\right)(y) d x=\int_{G / \Gamma} f(x) g(y x) d x
$$

for all $g \in L^{2}(G / \Gamma)$ and $y \in G / \Gamma$. We shall soon see that the image of $\bar{R}_{f}$ is indeed contained in $L^{2}(G / \Gamma)$. The assumption that $f \in C(G / \Gamma)$ ensures that $|f|$ is equal

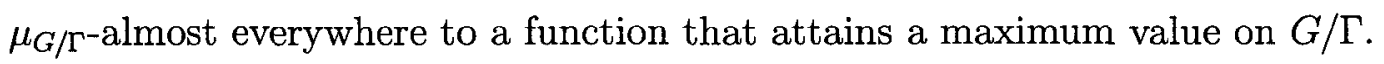

Proposition 4.1.2. For $f \in C(G / \Gamma)$, the function $\bar{R}_{f}$ is a bounded linear operator on $L^{2}(G / \Gamma)$.

Proof: Obviously, $\bar{R}_{f}$ is a linear map. For $g \in L^{2}(G / \Gamma)$, we check that in fact $\bar{R}_{f} g \in$ $L^{2}(G / \Gamma)$. Let $y \in G / \Gamma$. Note that $f \in C(G / \Gamma) \subseteq L^{\infty}(G / \Gamma)$. Define $h_{1}, h_{2}: G / \Gamma \rightarrow \mathbb{C}$ 
by $h_{1}(x)=f(x) g(y x)$ and $h_{2}(x)=1$ for all $x \in G / \Gamma$.

$$
\begin{aligned}
\int_{G / \Gamma}\left|h_{1}(x)\right|^{2} d x & =\int_{G / \Gamma}|f(x)|^{2}|g(y x)|^{2} d x \\
& \leqslant\|f\|_{\infty}^{2} \int_{G / \Gamma}|g(y x)|^{2} d x \\
& =\|f\|_{\infty}^{2} \int_{G / \Gamma}|g(x)|^{2} d x \\
& =\|f\|_{\infty}^{2}\|g\|_{2}^{2}<\infty \\
\int_{G / \Gamma}\left|h_{2}(x)\right|^{2} d x & =\int_{G / \Gamma} 1 d x \\
& =1<\infty
\end{aligned}
$$

and thus both $\left|h_{1}\right|^{2}$ and $\left|h_{2}\right|^{2}$ are integrable. Therefore, the assumptions of Hölder's inequality $([2], \S 1)$ are satisfied, so we have that

$$
\begin{aligned}
\left|\bar{R}_{f} g(y)\right| & =\left|\int_{G / \Gamma} f(x) g(y x) d x\right| \\
& \leqslant\left(\int_{G / \Gamma}|f(x) g(y x)|^{2} d x\right)^{\frac{1}{2}}\left(\int_{G / \Gamma} 1 d x\right)^{\frac{1}{2}} \\
& \leqslant\left(\|f\|_{\infty}^{2}\|g\|_{2}^{2}\right)^{\frac{1}{2}}(1)^{\frac{1}{2}} \\
& =\|f\|_{\infty}\|g\|_{2} .
\end{aligned}
$$

Therefore, the square $L^{2}$-norm of $\bar{R}_{f} g$ is

$$
\begin{aligned}
\left\|\bar{R}_{f} g\right\|_{2}^{2} & =\int_{G / \Gamma}\left|\bar{R}_{f} g(y)\right|^{2} d y \\
& \leqslant \int_{G / \Gamma}\left(\|f\|_{\infty}\|g\|_{2}\right)^{2} d y \\
& =\|f\|_{\infty}^{2}\|g\|_{2}^{2} \int_{G / \Gamma} 1 d y \\
& =\|f\|_{\infty}^{2}\|g\|_{2}^{2} \\
& <\infty .
\end{aligned}
$$


Thus $\bar{R}_{f} g \in L^{2}(G / \Gamma)$. This also shows that $\left\|\bar{R}_{f} g\right\|_{2} \leqslant\|f\|_{\infty}\|g\|_{2}$, proving that $\left\|\bar{R}_{f}\right\| \leqslant$ $\|f\|_{\infty}$. Therefore $\bar{R}_{f}$ is a bounded linear operator on $L^{2}(G / \Gamma)$.

Let $\mathcal{H}$ be a separable Hilbert space with inner product $\langle\cdot, \cdot\rangle$. An operator $A \in \mathcal{L}(\mathcal{H})$ is called positive if $\langle A x, x\rangle \geqslant 0$ for every $x \in \mathcal{H}$. For a positive operator $A \in \mathcal{L}(\mathcal{H})$, there exists a unique positive operator $B \in \mathcal{L}(\mathcal{H})$ such that $B^{2}=A([21], \S 6.4)$. The positive operator $B$ is called the square root of $A$, and denoted by $\sqrt{A}$. For every $A \in \mathcal{L}(\mathcal{H})$, the operator $A^{*} A$ is positive $([21], \S 6.4)$. Let $|A|=\sqrt{A^{*} A}$.

Fix an orthonormal basis $\left(e_{n}\right)_{n=1}^{\infty}$ of $\mathcal{H}$. For a positive operator $A \in \mathcal{L}(\mathcal{H})$, it is mentioned in ([21], §6.6) that if $\sum_{n=1}^{\infty}\left\langle A e_{n}, e_{n}\right\rangle$ converges, it is independent of the chosen orthonormal basis. A bounded linear operator $A \in \mathcal{L}(\mathcal{H})$ is called trace class if $\sum_{n=1}^{\infty}\left\langle|A| e_{n}, e_{n}\right\rangle<\infty$. The set of all trace class operators is denoted by $\mathcal{I}_{1}$. For $A \in \mathcal{I}_{1}$, the trace of $A$, denoted $\operatorname{tr}(A)$, is defined by

$$
\operatorname{tr}(A)=\sum_{n=1}^{\infty}\left\langle A e_{n}, e_{n}\right\rangle
$$

For $A \in \mathcal{I}_{1}$, the trace of $A$ is an absolutely convergent sum and is independent of the chosen orthonormal basis ([21], Thm VI.24).

That $\widehat{G / \Gamma}$ forms an orthonormal Hilbert basis for $L^{2}(G / \Gamma)$ was shown in $\S 2.2$. We will determine the trace of $\bar{R}_{f}$ using this orthonormal basis, should $\bar{R}_{f}$ be trace class. Recall the definition of the Fourier transform $\hat{f}$ of $f \in L^{1}(G / \Gamma)$ from $\S 3.2$ given by

$$
\hat{f}(\varphi)=\int_{G / \Gamma} f(x \Gamma) \overline{\varphi(x \Gamma)} d(x \Gamma)
$$

where $\varphi \in \widehat{G / \Gamma}$. As well, recall from $\S 2.2$ that $\varphi^{-1}=\bar{\varphi}$ for every $\varphi \in \widehat{G / \Gamma}$. 
Lemma 4.1.3. $\bar{R}_{f} \varphi=\hat{f}\left(\varphi^{-1}\right) \varphi$ for every $f \in C(G / \Gamma)$ and $\varphi \in \widehat{G / \Gamma}$.

Proof: Suppose $y \in G / \Gamma$ and $\varphi \in \widehat{G / \Gamma}$. Then

$$
\begin{aligned}
\bar{R}_{f} \varphi(y) & =\int_{G / \Gamma} f(x) \varphi(y x) d x \\
& =\left(\int_{G / \Gamma} f(x) \varphi(x) d x\right) \varphi(y) \\
& =\left(\int_{G / \Gamma} f(x) \overline{\varphi^{-1}(x)} d x\right) \varphi(y \Gamma) \\
& =\hat{f}\left(\varphi^{-1}\right) \varphi(y)
\end{aligned}
$$

Thus $\bar{R}_{f} \varphi=\hat{f}\left(\varphi^{-1}\right) \varphi$.

Lemma 4.1.3 shows that $\bar{R}_{f}$ is in fact diagonalized by $\widehat{G / \Gamma}$.

Theorem 4.1.4. If $f \in C(G / \Gamma)$, then the trace of $\bar{R}_{f}$ is given by

$$
\operatorname{tr}\left(\bar{R}_{f}\right)=\sum_{\varphi \in \widehat{G / \Gamma}} \hat{f}(\varphi)
$$

whenever $\bar{R}_{f}$ is trace class. 
Proof: Since $\widehat{G / \Gamma}$ is an orthonormal basis for $L^{2}(G / \Gamma)$, we have

$$
\begin{array}{rlr}
\operatorname{tr}\left(\bar{R}_{f}\right) & =\sum_{\varphi \in \widehat{G / \Gamma}}\left\langle\bar{R}_{f} \varphi, \varphi\right\rangle & \text { by }(4.3) \\
& =\sum_{\varphi \in \widehat{G / \Gamma}}\left\langle\hat{f}\left(\varphi^{-1}\right) \varphi, \varphi\right\rangle & \text { by Lemma } 4.1 .3 \\
& =\sum_{\varphi \in \widehat{G / \Gamma}} \hat{f}\left(\varphi^{-1}\right)\langle\varphi, \varphi\rangle \\
& =\sum_{\varphi \in \widehat{G / \Gamma}} \hat{f}\left(\varphi^{-1}\right) \\
& =\sum_{\varphi \in \widehat{G / \Gamma}} \hat{f}(\varphi) .
\end{array}
$$

We now have a formula for the trace of $\bar{R}_{f}$, should $\bar{R}_{f}$ be trace class. We would like to find all functions $f \in C(G / \Gamma)$ such that $\bar{R}_{f}$ is trace class.

Theorem 4.1.5. Let $f \in C(G / \Gamma)$. Then $\bar{R}_{f}$ is trace class if and only if $\hat{f} \in l_{1}(G / \Gamma)$.

Proof: Since $\bar{R}_{f}$ is diagonalized by $\widehat{G / \Gamma}$ (Lemma 4.1.3), we have that $\left|\bar{R}_{f}\right|(\varphi)=$ $\left|\widehat{f}\left(\varphi^{-1}\right)\right| \varphi$ for every $\varphi \in \widehat{G / \Gamma}$. Therefore, we can see that

$$
\begin{aligned}
\sum_{\varphi \in \widehat{G / \Gamma}}\left\langle\left|\bar{R}_{f}\right|(\varphi), \varphi\right\rangle & =\sum_{\varphi \in \widehat{G / T}}\left\langle\hat{f}\left(\varphi^{-1}\right) \mid \varphi, \varphi\right\rangle \\
& =\sum_{\varphi \in \widehat{G / \Gamma}}\left|\hat{f}\left(\varphi^{-1}\right)\right|\langle\varphi, \varphi\rangle \\
& =\sum_{\varphi \in \widehat{G / \Gamma}}\left|\hat{f}\left(\varphi^{-1}\right)\right| \\
& =\sum_{\varphi \in \widehat{G / \Gamma}}|\widehat{f}(\varphi)|
\end{aligned}
$$


By definition, $\bar{R}_{f}$ is trace class if and only if the sum $\sum_{n=1}^{\infty}\left\langle\left|\bar{R}_{f}\right|(\varphi), \varphi\right\rangle$ converges. As the topology of uniform convergence on compact set on $\widehat{G / \Gamma}$ is discrete (Proposition 2.2.1), we have

$$
\sum_{\varphi \in \widehat{G / \Gamma}}|\widehat{f}(\varphi)|=\int_{\widehat{G / \Gamma}}|\hat{f}(\varphi)| d(\varphi)
$$

where integration is taken with respect to the counting measure. This integral converges if and only if $\hat{f} \in l_{1}(G / \Gamma)$.

\section{Example:}

Let $G=\mathbb{R}$, under the usual topology and Lebesgue measure, and $\Gamma=\mathbb{Z}$. Take $f \in C(\mathbb{R} / \mathbb{Z})$ to be $f(x+\mathbb{Z})=1$ for all $x+\mathbb{Z} \in \mathbb{R} / \mathbb{Z}$. Let $y+\mathbb{Z} \in \mathbb{R} / \mathbb{Z}$ and $g \in L^{2}(\mathbb{R} / \mathbb{Z})$. Then

$$
\begin{aligned}
\left(\bar{R}_{f} g\right)(y+\mathbb{Z}) & =\int_{\mathbb{R} / \mathbb{Z}} f(x+\mathbb{Z}) g(y+x+\mathbb{Z}) d(x+\mathbb{Z}) \\
& =\int_{\mathbb{R} / \mathbb{Z}} g(y+x+\mathbb{Z}) d(x+\mathbb{Z}) \\
& =\int_{\mathbb{R} / \mathbb{Z}} g(x+\mathbb{Z}) d(x+\mathbb{Z}) \\
& =\int_{0}^{1} g(x+\mathbb{Z}) d x .
\end{aligned}
$$

Therefore, $\overline{R_{f}} g$ is a constant function on $\mathbb{R} / \mathbb{Z}$. In Chapter 2 , we discussed that the circle group $\mathbb{T}$ is homeomorphic to $\mathbb{R} / \mathbb{Z}$, and what the set of continuous characters of $\widehat{\mathbb{T}}$ is. Using this, we see that the group of continuous characters on $\widehat{\mathbb{R} / \mathbb{Z}}$ are of the form $\varphi_{k}$, where $k \in \mathbb{Z}$, and $\varphi_{k}$ is defined by $\varphi_{k}(x+\mathbb{Z})=e^{2 \pi i k x}$. The Fourier transform $\hat{f}$ applied to $\varphi_{k}$ is

$$
\begin{aligned}
\widehat{f}\left(\varphi_{k}\right) & =\int_{\mathbb{R} / \mathbb{Z}} f(x+\mathbb{Z}) e^{-2 \pi i k x} d(x+\mathbb{Z}) \\
& =\int_{0}^{1} e^{-2 \pi i k x} d x
\end{aligned}
$$


This is zero when $k \neq 0$ and one when $k=0$. Therefore, by Theorem 4.1.4, we have

$$
\operatorname{tr}\left(\bar{R}_{f}\right)=\sum_{k \in \mathbb{Z}} \hat{f}\left(\varphi_{k}\right)=1
$$

\subsection{A Related Representation of $G$}

We now define a representation $R$ of $G$ which is related to the right regular representation of $G / \Gamma$ from $\S 4.1$. In fact, we will see that the restriction of $R$ to a coset $x \Gamma$ will be a constant function, with constant value $\bar{R}(x \Gamma)$. Why do we introduce $R$ if it is so similar to $\bar{R}$ from $\S 4.1$ ? We do so in order to discuss the concepts from the previous section using the framework of the Poisson Summation Formula (§3.2). For $f \in C_{c}(G)$, we will define the linear operator $R_{f}$ on $L^{2}(G / \Gamma)$ and prove that is is equal to $\bar{R}_{f_{\Gamma}}$, where $f_{\Gamma}$ is the $\Gamma$-projection defined in $\S 3.2$. We will prove that $\operatorname{tr}\left(R_{f}\right)=\sum_{\gamma \in \Gamma} f(\gamma)$ whenever $R_{f}$ is trace class, and provide an example which demonstrates this trace computation.

Let $R: G \rightarrow \mathcal{U}\left(L^{2}(G / \Gamma)\right)$ be defined by

$$
R(x)=R_{x}=\bar{R}_{x \Gamma}
$$

for all $x \in G$. That $R$ is a unitary representation of $G$ follows from $\bar{R}$ being a unitary representation of $G / \Gamma$.

For $f \in C_{c}(G)$, let $R_{f}: L^{2}(G / \Gamma) \rightarrow L^{2}(G / \Gamma)$ be defined by

$$
\left(R_{f} g\right)(y \Gamma)=\int_{G} f(x)\left(R_{x} g\right)(y \Gamma) d x=\int_{G} f(x) g(y x \Gamma) d x
$$


for $g \in L^{2}(G / \Gamma)$ and $y \Gamma \in G / \Gamma$. We shall see shortly that in fact the image of $R_{f}$ is inside of $L^{2}(G / \Gamma)$. The next theorem describes the relationship between $R_{f}$ and $\bar{R}_{f_{\Gamma}}$.

Theorem 4.2.1. For $f \in C_{c}(G)$, we have $R_{f}=\bar{R}_{f_{\Gamma}}$.

Proof: Let $g \in L^{2}(G / \Gamma)$ and $y \Gamma \in G / \Gamma$. Recall from $\S 3.1$ that Theorem 3.1.6 also holds for functions in $L^{1}(G)$. Therefore we have that

$$
\begin{aligned}
\left(R_{f} g\right)(y \Gamma) & =\int_{G} f(x) g(y x \Gamma) d x \\
& =\int_{G / \Gamma} \sum_{\gamma \in \Gamma} f(x \gamma) g(y x \gamma \Gamma) d(x \Gamma) \quad \text { by Theorem 3.1.6 } \\
& =\int_{G / \Gamma} f_{\Gamma}(x \Gamma) g(y x \Gamma) d(x \Gamma) \\
& =\left(\bar{R}_{f_{\Gamma}} g\right)(y \Gamma)
\end{aligned}
$$

This shows that $R_{f} g=\bar{R}_{f_{\Gamma}} g$ for all $g \in L^{2}(G / \Gamma)$, proving that $R_{f}=\bar{R}_{f_{\Gamma}}$.

Corollary 4.2.2. For $f \in C_{c}(G)$, the function $R_{f}$ is a bounded linear operator on $L^{2}(G / \Gamma)$.

Proof: As $f_{\Gamma} \in C(G / \Gamma)$ was proven in Theorem 3.1.4, we know that $\bar{R}_{f_{\Gamma}}$ is a bounded linear operator on $L^{2}(G / \Gamma)$ by Proposition 4.1.2. The result follows from Theorem 4.2.1.

Corollary 4.2.3. Suppose $f \in C_{c}(G)$ and $\varphi \in \widehat{G / \Gamma}$. Then $R_{f} \varphi=\widehat{f}_{\Gamma}\left(\varphi^{-1}\right) \varphi$. 
Proof: By Theorem 4.2.1, $R_{f}=\bar{R}_{f_{\Gamma}}$. The result now follows from Lemma 4.1.3.

Corollary 4.2.4. For $f \in C_{c}(G)$, we have

$$
\operatorname{tr}\left(\bar{R}_{f_{\Gamma}}\right)=\operatorname{tr}\left(R_{f}\right)=\sum_{\gamma \in \Gamma} f(\gamma)
$$

whenever $\bar{R}_{f_{\mathrm{r}}}=R_{f}$ is trace class.

Proof: Recall from $\S 3.2$ that, for $\varphi \in \widehat{G / \Gamma}, \varphi^{\prime} \in \widehat{G}$ is defined by $\varphi^{\prime}(x)=\varphi(x \Gamma)$ for all $x \in G$. Using Theorem 4.1.4, Lemma 3.2.2 and the Poisson Summation Formula (Theorem 3.2.3), we have

$$
\begin{array}{rlr}
\operatorname{tr}\left(R_{f}\right) & =\operatorname{tr}\left(\bar{R}_{f_{\Gamma}}\right) & \\
& =\sum_{\varphi \in \widehat{G / \Gamma}} \hat{f}_{\Gamma}(\varphi) & \text { by Theorem } 4.1 .4 \\
& =\sum_{\varphi \in \widehat{G / \Gamma}} \hat{f}\left(\varphi^{\prime}\right) & \text { by Lemma } 3.2 .2 \\
& =\sum_{\gamma \in \Gamma} f(\gamma) . & \text { by the Poisson Summation Formula }
\end{array}
$$

Note that this sum only converges when the Poisson Summation Formula is valid, which is certainly true when $\hat{f}_{\Gamma} \in l^{1}(\widehat{G / \Gamma})$, which we proved in Theorem 4.1.5 is equivalent to $\bar{R}_{f_{\Gamma}}$ being trace class. 


\section{Example:}

Consider $G=\mathbb{R}$ and $\Gamma=\mathbb{Z}$. Define $f \in C_{c}(\mathbb{R})$ by

$$
f(x)= \begin{cases}1+x & \text { if }-1 \leqslant x \leqslant 0 \\ 1-x & \text { if } 0<x \leqslant 1 \\ 0 & \text { otherwise }\end{cases}
$$

For $y+\mathbb{Z} \in \mathbb{R} / \mathbb{Z}$ and $g \in L^{2}(G / \Gamma)$, we have that

$$
\begin{aligned}
\left(R_{f} g\right)(y+\mathbb{Z}) & =\int_{\mathbb{R}} f(x) g(y+x+\mathbb{Z}) d x \\
& =\int_{-1}^{0}(1+x) g(y+x+\mathbb{Z}) d x+\int_{0}^{1}(1-x) g(y+x+\mathbb{Z}) d x
\end{aligned}
$$

Set $z=1+x$

$$
=\int_{0}^{1}(z) g(y+z+\mathbb{Z}) d z+\int_{0}^{1}(1-x) g(y+x+\mathbb{Z}) d x
$$

Rename $z=x$

$$
\begin{aligned}
& =\int_{0}^{1}(x) g(y+x+\mathbb{Z}) d x+\int_{0}^{1}(1-x) g(y+x+\mathbb{Z}) d x \\
& =\int_{0}^{1} g(y+x+\mathbb{Z}) d x \\
& =\int_{0}^{1} g(x+\mathbb{Z}) d x .
\end{aligned}
$$

By Theorem 4.2.4, we know that $\operatorname{tr}\left(R_{f}\right)=\sum_{z \in \mathbb{Z}} f(z)=1$. Notice that $R_{f}$ is identical to $\bar{R}_{f}$ from our example in $\S 4.1$. This is because the $\mathbb{Z}$-projection $f_{\mathbb{Z}}$ on $\mathbb{R} / \mathbb{Z}$ is the constant function 1 , which is what we chose $f$ to be in that example. The trace values are the same since $\operatorname{tr}\left(R_{f}\right)=\operatorname{tr}\left(\bar{R}_{f_{\mathrm{Z}}}\right)$. 


\section{Chapter 5}

\section{Traces of Linear Operators on $L^{2}(G / \Gamma \rtimes \Theta)$}

Recall from Chapter 3 that $G$ is a second countable LCA group with a discrete subgroup $\Gamma$ such that $G / \Gamma$ is compact. In this chapter we consider a finite subgroup $\Theta$ of $\operatorname{Aut}(G)$ and use it to create the semi-direct products $G \rtimes \Theta$ and $G / \Gamma \rtimes \Theta$. We will define a representation $R$ of $G \rtimes \Theta$ and the (right)-regular representation $\bar{R}$ of $G / \Gamma \rtimes \Theta$, similar to what we did in Chapter 4. For $f \in C_{c}(G \rtimes \Theta)$, we will define the $\Gamma$-projection $f_{\Gamma}$ similar to as we did in Chapter 3 , and define bounded linear operators $R_{f}$ and $\bar{R}_{f_{\Gamma}}$ on $L^{2}(G / \Gamma \rtimes \Theta)$, which we shall see are equal to one another. Our goal is to determine the trace of these linear operators. In fact, Chapter 4 is the special case when $\Theta=\{i d\}$ is the trivial subgroup of $\operatorname{Aut}(G)$. It may not be true in all cases that $R_{f}$ is trace class. We will show, however, that $R_{f}$ is a Hilbert-Schmidt operator using integral kernels, and that $R_{f}$ is trace class when $f$ is the convolution of two functions in $C_{c}(G \rtimes \Theta)$. In this case, we will compute the trace of $R_{f}$, using its integral kernel and a theorem of Duflo ([4]). 


\subsection{An Extension of $G / \Gamma$ using a Semi-Direct Prod- uct}

We will define the semi-direct products $G / \Gamma \rtimes \Theta$ and $G \rtimes \Theta$, and prove that $G / \Gamma \rtimes \Theta$ is a topological group. That $G \rtimes \Theta$ is also a topological group follows in a similar way. Hence, the proof is omitted. We will construct a Haar measure on each of $G / \Gamma \rtimes \Theta$ and $G \rtimes \Theta$ using the measures $\mu_{G}$ and $\mu_{G / \Gamma}$ constructed in $\S 3.1$. These measures are both left and right Haar measures.

Consider $\operatorname{Aut}(G)$, the set of all automorphisms of the topological group $G$ which are homeomorphisms. The set $\operatorname{Aut}(G)$ forms a group under the group law of composition. Let $\Theta$ be a finite subgroup of $A u t(G)$ which preserves $\Gamma$. That is if $\theta \in \Theta$, then $\theta(\gamma) \in \Gamma$ for every $\gamma \in \Gamma$. Equip $\Theta$ with the discrete topology. By a remark made in $\S 2.1, \Theta$ is a compact topological group under this topology. Denote by $e_{\Theta}$ the identity element of $\Theta$.

The semi-direct product $G / \Gamma \rtimes \Theta$ is the set $\{(y \Gamma, \theta): y \Gamma \in G / \Gamma, \quad \theta \in \Theta\}$, which forms a group under multiplication defined by

$$
(y \Gamma, \theta)(x \Gamma, \tau)=(y \theta(x) \Gamma, \theta \tau)
$$

for all $(y \Gamma, \theta),(x \Gamma, \tau) \in G / \Gamma \rtimes \Theta$. This multiplication is well defined, since for $\gamma_{1}, \gamma_{2} \in$ $\Gamma$, we have that

$$
y \gamma_{1} \theta\left(x \gamma_{1}\right) \Gamma=y \theta(x) \gamma_{1} \theta\left(\gamma_{2}\right) \Gamma=y \theta(x) \Gamma
$$

Proposition 5.1.1. The group $G / \Gamma \rtimes \Theta$ equipped with the product topology ([23] §3.3) is a topological group. 
Proof: We will show this by proving the multiplication and inverse maps for $G / \Gamma \rtimes \Theta$ are continuous. Let $m_{G}: G \times G \rightarrow G$ and $i_{G}: G \rightarrow G$ be the multiplication and inverse maps of $G$, respectively. For $\theta \in \Theta$, we know $\theta^{-1} \in \Theta$, meaning $\theta^{-1}$ is continuous. Thus the map $\theta^{-1} \circ i_{G}$, which is $x \mapsto \theta^{-1}\left(x^{-1}\right)$, is also continuous. The map $x \Gamma \mapsto \theta^{-1}\left(x^{-1}\right) \Gamma$ will therefore be continuous in $G / \Gamma$. Since $\Theta$ is discrete, the map $\theta \mapsto \theta^{-1}$ is continuous. Therefore the inverse function of $G / \Gamma \rtimes \Theta$ given by $(x \Gamma, \theta) \mapsto(x \Gamma, \theta)^{-1}=\left(\theta^{-1}\left(x^{-1}\right) \Gamma, \theta^{-1}\right)$ is continuous. For $\theta \in \Theta$, let $T_{\theta}: G \times G \rightarrow$ $G \times G$ be given by:

$$
T_{\theta}(y, x)=(y, \theta(x))
$$

$T_{\theta}$ is continuous since $\theta$ is continuous. The map $(y, x) \mapsto y \theta(x)$ is the composition $m_{G} \circ T_{\theta}$, and thus is continuous. Therefore the map $(y \Gamma, x \Gamma) \mapsto y \theta(x) \Gamma$ is a continuous function from $G / \Gamma \times G / \Gamma$ to $G / \Gamma$. Since $(\theta, \tau) \mapsto \theta \tau$ is also continuous, the multiplication function of $G / \Gamma \rtimes \Theta$ given by $((y \Gamma, \theta),(x, \tau)) \mapsto(y \theta(x), \theta \tau)$ is continuous.

Since both $G / \Gamma$ and $\Theta$ are compact, the space $G / \Gamma \rtimes \Theta$ under the product topology is compact by Tychonoff's Theorem ([23], $\S 6.1)$. Since $G / \Gamma$ is a Hausdorff space, the set $\{\Gamma\}$ is closed in $G / \Gamma$, as mentioned in $\S 2.1$. As $\left\{e_{\Theta}\right\}$ is clearly closed in $\Theta$, the set $\left\{\left(\Gamma, e_{\Theta}\right)\right\}=\{\Gamma\} \times\left\{e_{\Theta}\right\}$ is closed in $G / \Gamma \rtimes \Theta$, showing that $G / \Gamma \rtimes \Theta$ is a Hausdorff space. Therefore $G / \Gamma \rtimes \Theta$ is a compact group. However, $G / \Gamma \rtimes \Theta$ need not be abelian.

We can similarly define the semi-direct product $G \rtimes \Theta$ of $G$ and $\Theta$ as the set $G \times \Theta$ under group multiplication

$$
(y, \theta)(x, \tau)=(y \theta(x), \theta \tau)
$$

for all $(y, \theta),(x, \tau) \in G \rtimes \Theta$. When equipped with the product topology, a similar argument shows that $G \rtimes \Theta$ is a topological group. In fact, $G \rtimes \Theta$ will be a locally compact group, but once again need not be abelian. If we consider the normal discrete 
subgroup $\Gamma \times\left\{e_{\Theta}\right\}$ of $G \rtimes \Theta$, the quotient space $(G \rtimes \Theta) /\left(\Gamma \times\left\{e_{\Theta}\right\}\right)$ is isomorphic to $G / \Gamma \rtimes \Theta$. In this sense, $G / \Gamma \rtimes \Theta$ can be viewed in the context of how we originally defined it or can be viewed as this quotient of $G \rtimes \Theta$.

We now define Haar measures on both $G \rtimes \Theta$ and $G / \Gamma \rtimes \Theta$. For a set $A \in \mathcal{B}(G / \Gamma \rtimes \Theta)$ and $\theta \in \Theta$, let $A_{\theta}=\{x \Gamma \in G / \Gamma:(x \Gamma, \theta) \in A\}$. It can be shown that the set $A_{\theta}$ is in $\mathcal{B}(G / \Gamma)$. Define $\mu_{G / \Gamma \rtimes \Theta}$ to be the measure on $\mathcal{B}(G / \Gamma \rtimes \Theta)$ defined by

$$
\mu_{G / \Gamma \rtimes \Theta}(A)=\sum_{\theta \in \Theta} \mu_{G / \Gamma}\left(A_{\theta}\right)
$$

for every $A \in \mathcal{B}(G / \Gamma \rtimes \Theta)$. That $\mu_{G / \Gamma \rtimes \Theta}$ is a non-zero Radon measure on $G / \Gamma \rtimes \Theta$ follows from the fact that $\mu_{G / \Gamma}$ is a non-zero Radon measure on $G / \Gamma$. We will soon see that this measure is in fact a left and right Haar measure. It can be shown that when $h$ is $\mu_{G / \Gamma \rtimes \Theta}$-integrable, then for each $\tau \in \Theta$ the function $h^{\tau}: G / \Gamma \rightarrow \mathbb{C}$ given

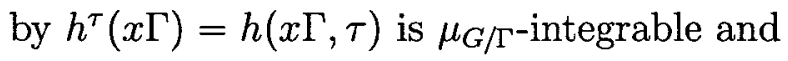

$$
\int_{G / \Gamma \times \Theta} h(x \Gamma, \tau) d(x \Gamma, \tau)=\sum_{\tau \in \Theta} \int_{G / \Gamma} h(x \Gamma, \tau) d(x \Gamma) .
$$

Typically we would normalize a Haar measure on a compact group to 1 . However, we have opted against this in order to have this simple relationship between $\mu_{G / \Gamma \rtimes \Theta}$ and $\mu_{G / \Gamma}$. Note that by our definition we have $\mu_{G / \Gamma \rtimes \Theta}(G / \Gamma \rtimes \Theta)=|\Theta|$.

Lemma 5.1.2. For $\theta \in \Theta$ and $f \in L^{1}(G)$, we have

$$
\int_{G} f(\theta(x)) d x=\int_{G} f(x) d x
$$


Proof: Note that the integral $\int_{G} f(\theta(x)) d x$ is a Haar integral, which means for $y \in G$

$$
\begin{aligned}
\int_{G} f(y \theta(x)) d x & =\int_{G} f\left(\theta\left(\theta^{-1}(y) x\right)\right) d x \\
& =\int_{G} f(\theta(x)) d x
\end{aligned}
$$

which follows from the invariance of $\mu_{G}$.

We have two Haar integrals on locally compact group $G$, so they are the same up to scaling ( $\S 2.1$ ). That is for some positive number $\alpha>0$, we have

$$
\int_{G} f(\theta(x)) d x=\alpha \int_{G} f(x) d x
$$

This implies that $\int_{G} f\left(\theta^{j}(x)\right) d x=\alpha^{j} \int_{G} f(x) d x$ for all $j \geqslant 1$. Taking $j=|\theta|$ we get $\alpha^{j}=1$. Therefore $\alpha=1$, so we have

$$
\int_{G} f(\theta(x)) d x=\int_{G} f(x) d x
$$

Lemma 5.1.3. For $\theta \in \Theta$ and $f \in L^{1}(G / \Gamma)$, we have

$$
\int_{G / \Gamma} f(\theta(x) \Gamma) d(x \Gamma)=\int_{G / \Gamma} f(x \Gamma) d(x \Gamma)
$$

Proposition 5.1.4. The measure $\mu_{G / \Gamma \rtimes \Theta}$ is a left Haar measure on $G / \Gamma \rtimes \Theta$.

Proof: To prove that $\mu_{G / \Gamma \rtimes \Theta}$ is a left Haar measure, it suffices to show for a $\mu_{G / \Gamma \rtimes \Theta^{-}}$ integrable function $f$ on $G / \Gamma \rtimes \Theta$ that

$$
\int_{G / \Gamma \rtimes \Theta} f((y \Gamma, \theta)(x \Gamma, \tau)) d(x \Gamma, \tau)=\int_{G / \Gamma \rtimes \Theta} f(x \Gamma, \tau) d(x \Gamma, \tau) .
$$


Suppose that $(y \Gamma, \theta) \in G / \Gamma \rtimes \Theta$. Then

$$
\begin{aligned}
\int_{G / \Gamma \rtimes \Theta} f((y \Gamma, \theta)(x \Gamma, \tau)) d(x \Gamma, \tau) & =\sum_{\tau \in \Theta} \int_{G / \Gamma} f(y \theta(x) \Gamma, \theta \tau) d(x \Gamma) & \\
& =\sum_{\tau \in \Theta} \int_{G / \Gamma} f(y x \Gamma, \theta \tau) d(x \Gamma) & \text { By Corollary 5.1.3 } \\
& =\sum_{\tau \in \Theta} \int_{G / \Gamma} f(x \Gamma, \theta \tau) d(x \Gamma) & \\
& =\sum_{\sigma \in \Theta} \int_{G / \Gamma} f(x \Gamma, \sigma) d(x \Gamma) & \text { Set } \sigma=\theta \tau \\
& =\int_{G / \Gamma \rtimes \Theta} f(x \Gamma, \tau) d(x \Gamma, \tau) . & \text { Renaming } \sigma=\tau
\end{aligned}
$$

Since all compact groups are unimodular $([7], \S 2.4)$, we also have that $\mu_{G / \Gamma \rtimes \Theta}$ is a right Haar measure.

In an almost identical way, we can define a Haar measure $\mu_{G \rtimes \Theta}$ on $G \rtimes \Theta$, where it can once again be shown that when $h$ is $\mu_{G \rtimes \Theta}$-integrable, the similarly defined $h^{\tau}$ is $\mu_{G}$-integrable for all $\tau \in \Theta$ and

$$
\int_{G \rtimes \Theta} h(x, \tau) d(x, \tau)=\sum_{\tau \in \Theta} \int_{G} h(x, \tau) d x
$$

\subsection{The Representation $R$ of $G \rtimes \Theta$}

We will define a representation $R$ of $G \rtimes \Theta$, but omit the proof that it is indeed a representation, as it follows in a similar way to work done in $\S 4$.1. For $f \in C_{c}(G \rtimes \Theta)$, we will define a bounded linear operator $R_{f}$ on $L^{2}(G / \Gamma \rtimes \Theta)$. Next we will extend the 
definition of the $\Gamma$-projection $f_{\Gamma}$ for a function $f \in C_{c}(G)$ from $\S 3.2$ to the semi-direct product, and prove that the $\Gamma$-projection of $f \in C_{c}(G \rtimes \Theta)$ is once again continuous.

Let $R: G \rtimes \Theta \rightarrow \mathcal{U}\left(L^{2}(G / \Gamma \rtimes \Theta)\right)$ be defined by

$$
(R(x, \tau) g)(y \Gamma, \theta)=g((y \Gamma, \theta)(x \Gamma, \tau))=g(y \theta(x) \Gamma, \theta \tau)
$$

for all $(x, \tau) \in G \rtimes \Theta, g \in L^{2}(G / \Gamma \rtimes \Theta)$ and $(y \Gamma, \theta) \in G / \Gamma \rtimes \Theta$. The function $R$ is a unitary representation of $G \rtimes \Theta$. This can be shown by proving that $R$ is continuous and a homomorphism, both of which follow in a very similar way to the proof that $\bar{R}$ is a unitary representation of $G / \Gamma$ (Proposition 4.1.1). In fact, $R$ is the composition of the right regular representation of $G / \Gamma \rtimes \Theta$ with the projection $\pi: G \rtimes \Theta \rightarrow G / \Gamma \rtimes \Theta$.

For $f \in C_{c}(G \rtimes \Theta)$, define $R_{f}: L^{2}(G / \Gamma \rtimes \Theta) \rightarrow L^{2}(G / \Gamma \rtimes \Theta)$ by

$$
\begin{aligned}
\left(R_{f} g\right)(y \Gamma, \theta) & =\int_{G \rtimes \Theta} f(x, \tau)(R(x, \tau) g)(y \Gamma, \theta) d(x, \tau) \\
& =\int_{G \rtimes \Theta} f(x, \tau) g(y \theta(x) \Gamma, \theta \tau) d(x, \tau) .
\end{aligned}
$$

for all $g \in L^{2}(G / \Gamma \rtimes \Theta)$ and all $(y \Gamma, \theta) \in G / \Gamma \rtimes \Theta$. Note that the space $C_{c}(G \rtimes \Theta)$ is in actuality the subspace of $L^{2}(G \rtimes \Theta)$ of functions equal $\mu_{G \rtimes \Theta}$-almost everywhere to a continuous function of compact support.

The proof that $R_{f}$ is a bounded linear operator on $L^{2}(G / \Gamma \rtimes \Theta)$ is almost identical to the proof of Proposition 4.1.2 from $\S 4.1$, and hence is omitted.

For $f \in C_{c}(G \rtimes \Theta)$, define the $\Gamma$-projection of $f$, denoted $f_{\Gamma}: G / \Gamma \rtimes \Theta \rightarrow \mathbb{C}$, by

$$
f_{\Gamma}(y \Gamma, \theta)=\sum_{\gamma \in \Gamma} f(y \gamma, \theta)
$$


Theorem 5.2.1. The $\Gamma$-projection $f_{\Gamma}$ is continuous.

Proof: For $\theta \in \Theta$, let $f^{\theta}: G \rightarrow \mathbb{C}$ be given by $f^{\theta}(x)=f(x, \theta)$. We want to show that $f^{\theta}$ is a continuous, compactly supported function on $G$. Once this is shown, then we can apply Theorem 3.1.4 and conclude that $f_{\Gamma}^{\theta}$ is continuous.

Recall that supp $=\overline{\{(x, \theta) \in G \rtimes \Theta: f(x, \theta) \neq 0\}}$, and is a compact set. Consider supp $f^{\theta}=\overline{\left\{x \in G: f^{\theta}(x) \neq 0\right\}}$, and define $H^{\theta}=\left\{(x, \theta): x \in \operatorname{supp}^{\theta}\right\}$. Since $\Theta$ is discrete, there is a topological isomorphism between supp $f^{\theta}$ and $H^{\theta}$, implying that $H^{\theta}$ is closed. Since $H^{\theta}$ is a closed subset of the compact set suppf, $H^{\theta}$ is compact, which implies that suppf $f^{\theta}$ is compact. Thus $f^{\theta}$ is a function of compact support.

Suppose $U$ is an open subset of $\mathbb{C}$. Since $f$ is continuous, $f^{-1}(U)$ is open in $G \rtimes \Theta$. Let $y \in\left(f^{\theta}\right)^{-1}(U)$ be given. Then $(y, \theta) \in f^{-1}(U)$, so there exists an open $U_{\theta} \subseteq G$ such that $(y, \theta) \in U_{\theta} \times\{\theta\} \subseteq f^{-1}(U)$. Then clearly $U_{\theta} \subseteq\left(f^{\theta}\right)^{-1}(U)$. So for all $y \in\left(f^{\theta}\right)^{-1}(U)$, there exists open $U_{\theta} \subseteq G$ such that $y \in U_{\theta} \subseteq\left(f^{\theta}\right)^{-1}(U)$. Thus $\left(f^{\theta}\right)^{-1}(U)$ is open in $G$, implying $f^{\theta}$ is continuous. Therefore we have shown that for all $\theta \in \Theta, f^{\theta}$ is in $C_{c}(G)$.

For $(y \Gamma, \theta) \in G / \Gamma \rtimes \Theta$, we have that

$$
f_{\Gamma}^{\theta}(y \Gamma)=\sum_{\gamma \in \Gamma} f^{\theta}(y \gamma)=\sum_{\gamma \in \Gamma} f(y \gamma, \theta)=f_{\Gamma}(y \Gamma, \theta)
$$

Let $U$ be open in $\mathbb{C}$. The above equality implies that $f_{\Gamma}^{-1}(U)=\cup_{\theta \in \Theta}\left(\left(f_{\Gamma}^{\theta}\right)^{-1}(U), \theta\right)$. By the continuity of $f_{\Gamma}^{\theta},\left(\left(f_{\Gamma}^{\theta}\right)^{-1}(U), \theta\right)$ is open in $G / \Gamma \rtimes \Theta$ for all $\theta \in \Theta$, meaning $f_{\Gamma}^{-1}(U)$ is open in $G / \Gamma \rtimes \Theta$. This proves that $f_{\Gamma}$ is continuous. 
Viewing the group $G / \Gamma \rtimes \Theta$ as the quotient $(G \rtimes \Theta) /\left(\Gamma \times\left\{e_{\Theta}\right\}\right)$, we can apply ([7], Prop 2.48) to get that, for every $g \in C(G / \Gamma \rtimes \Theta)$, there is a function $f \in C_{c}(G \rtimes \Theta)$ such that $f_{\Gamma}=g$. In other words, the $\Gamma$-projection function $P: C_{c}(G \rtimes \Theta) \rightarrow$ $C(G / \Gamma \rtimes \Theta)$ given by $P(f)=f_{\Gamma}$ is surjective.

\subsection{Computing the Integral Kernel}

We will define what it is for a linear operator on a separable Hilbert space to be Hilbert-Schmidt. Using a theorem proved in [21], we will show that for every $f \in C_{c}(G \rtimes \Theta)$, the linear operator $R_{f}$ is a Hilbert-Schmidt operator. This will be accomplished by finding the integral kernel $K_{f}$ of $R_{f}$.

Take $\mathcal{H}$ to be a separable Hilbert space with inner product $\langle\cdot, \cdot\rangle$. Recall from $\S 4.1$ that, for an orthonormal basis $\left(e_{n}\right)_{n=1}^{\infty}$ of $\mathcal{H}$ and $A \in \mathcal{L}(\mathcal{H})$, the trace of $A$ is given by $\operatorname{tr}(A)=\sum_{n=1}^{\infty}\left\langle A e_{n}, e_{n}\right\rangle$, should $A$ be trace class. A bounded linear operator $A \in \mathcal{L}(\mathcal{H})$ is called Hilbert-Schmidt if $\operatorname{tr}\left(A^{*} A\right)<\infty$. The family of Hilbert-Schmidt operators is denoted by $\mathcal{I}_{2}$.

Recall that $\mathcal{I}_{1}$ denotes the space of trace class operators defined in $\S 4.1$. Both $\mathcal{I}_{1}$ and $\mathcal{I}_{2}$ are subspaces of $\mathcal{L}(\mathcal{H})$, and $A \in \mathcal{I}_{1}$ if and only if there exists $B, C \in \mathcal{I}_{2}$ such that $A=B C([21], \S 6.6)$. The next theorem is stated and proved in ([21], §6.6).

Theorem 5.3.1. Let $(M, \mu)$ be a measure space and $\mathcal{H}=L^{2}(M, \mu)$ be separable. Then $A \in \mathcal{L}(\mathcal{H})$ is Hilbert-Schmidt if and only if there exists a function $K \in L^{2}(M \times M)$ with

$$
(A g)(x)=\int_{M} K(x, y) g(y) d \mu(y) .
$$

for all $g \in \mathcal{H}$ and for $\mu$-almost everywhere $x \in M$. 
The function $K$ is called the integral kernel of $A$. Note that $L^{2}(M, \mu)$ is separable if and only if $M$ is second countable ([8], Thm 24.14). As $G / \Gamma \rtimes \Theta$ is the product of two second countable spaces, it is also second countable. Thus, we can apply this theorem for $L^{2}(G / \Gamma \rtimes \Theta)$.

We would like to show that, for $f \in C_{c}(G \rtimes \Theta)$, the linear operator $R_{f}$ is a HilbertSchmidt operator. By Theorem 5.3.1, we can show this by proving the existence of an integral kernel for $R_{f}$. We will need to perform a change of variable in order to compute the integral kernel. Note that with the substitution $z=y \theta(x)$, we have $d z=d(y \theta(x))=d(\theta(x))=d x$ (by Lemma 5.1.2).

$$
\begin{array}{rlr}
\left(R_{f} g\right)(y \Gamma, \theta) & =\int_{G \rtimes \Theta} f(x, \tau) g(y \theta(x) \Gamma, \theta \tau) d(x, \tau) & \\
& =\sum_{\tau \in \Theta} \int_{G} f(x, \tau) g(y \theta(x) \Gamma, \theta \tau) d x & \\
& =\sum_{\beta \in \Theta} \int_{G} f\left(x, \theta^{-1} \beta\right) g(y \theta(x) \Gamma, \beta) d x \\
& =\sum_{\beta \in \Theta} \int_{G} f\left(\theta^{-1}\left(y^{-1} z\right), \theta^{-1} \beta\right) g(z \Gamma, \beta) d z & z=y \theta(x) \\
& =\sum_{\beta \in \Theta} \int_{G / \Gamma}\left[\sum_{\gamma \in \Gamma} f\left(\theta^{-1}\left(y^{-1} \gamma z\right), \theta^{-1} \beta\right) g(\gamma z \Gamma, \beta)\right] d(z \Gamma) & \\
& =\int_{G / \Gamma \rtimes \Theta}\left[\sum_{\gamma \in \Gamma} f\left(\theta^{-1}\left(y^{-1} \gamma z\right), \theta^{-1} \beta\right)\right] g(z \Gamma, \beta) d(z \Gamma, \beta) .
\end{array}
$$

We define the function $K_{f}:(G / \Gamma \rtimes \Theta) \times(G / \Gamma \rtimes \Theta) \rightarrow \mathbb{C}$ by

$$
K_{f}(y \Gamma, \theta, z \Gamma, \beta)=\sum_{\gamma \in \Gamma} f\left(\theta^{-1}\left(y^{-1} \gamma z\right), \theta^{-1} \beta\right)
$$

We will show that $K_{f}$ is the integral kernel of $R_{f}$. According to Theorem 5.3.1, this is shown by proving that $K_{f} \in L^{2}(G / \Gamma \rtimes \Theta \times G / \Gamma \rtimes \Theta)$, which will also prove that 
$R_{f}$ is a Hilbert-Schmidt operator. Since $\theta^{-1}$ preserves $\Gamma$, we have that

$$
\begin{aligned}
K_{f}(y \Gamma, \theta, z \Gamma, \beta) & =\sum_{\gamma \in \Gamma} f\left(\theta^{-1}\left(y^{-1} \gamma z\right), \theta^{-1} \beta\right) \\
& =\sum_{\gamma \in \Gamma} f\left(\theta^{-1}\left(y^{-1} z\right) \theta^{-1}(\gamma), \theta^{-1} \beta\right) \\
& =\sum_{\gamma \in \Gamma} f\left(\theta^{-1}\left(y^{-1} z\right) \gamma, \theta^{-1} \beta\right) \\
& =f_{\Gamma}\left(\theta^{-1}\left(y^{-1} z\right) \Gamma, \theta^{-1} \beta\right)
\end{aligned}
$$

Theorem 5.3.2. For $f \in C_{c}(G \rtimes \Theta)$, the integral kernel $K_{f}: G / \Gamma \rtimes \Theta \times G / \Gamma \rtimes \Theta \rightarrow \mathbb{C}$ is continuous.

Proof: Since $G / \Gamma \rtimes \Theta$ is a topological group, the inverse function defined by $i_{G / \Gamma \rtimes \Theta}(y \Gamma, \theta)=$ $\left(\theta^{-1}\left(y^{-1}\right) \Gamma, \theta^{-1}\right)$, and multiplication function defined by $m_{G / \Gamma \times \Theta}((y \Gamma, \theta),(z \Gamma, \beta))=$ $(y \theta(z) \Gamma, \theta \beta)$ are continuous. We have that $\left(\theta^{-1}\left(y^{-1} z\right) \Gamma, \theta^{-1} \beta\right)=\left(\theta^{-1}\left(y^{-1}\right) \Gamma, \theta^{-1}\right)(z \Gamma, \beta)$, so the function $p: G / \Gamma \rtimes \Theta \times G / \Gamma \rtimes \Theta \rightarrow G / \Gamma \rtimes \Theta$ defined by $p(y \Gamma, \theta, z \Gamma, \beta)=$ $\left(\theta^{-1}\left(y^{-1} z\right) \Gamma, \theta^{-1} \beta\right)$ is the function $m_{G / \Gamma \rtimes \Theta} \circ i_{G / \Gamma \rtimes \Theta}$, which is the composition of two continuous functions. Thus $p$ is continuous. Since $K_{f}(y \Gamma, \theta, h \Gamma, z)=f_{\Gamma}(p(y \Gamma, \theta, h \Gamma, z))$ and $f_{\Gamma}$ is continuous by Theorem 5.2.1, we know that $K_{f}$ is also continuous.

Corollary 5.3.3. For $f \in C_{c}(G \rtimes \Theta)$, the integral kernel $K_{f}$ of $R_{f}$ is in $L^{2}((G / \Gamma \rtimes \Theta) \times(G / \Gamma \rtimes \Theta))$. 
Proof: Since the group $G / \Gamma \rtimes \Theta \times G / \Gamma \rtimes \Theta$ is compact, by Lemma 2.2.5, we know that $C(G / \Gamma \rtimes \Theta \times G / \Gamma \rtimes \Theta) \subseteq L^{2}(G / \Gamma \rtimes \Theta \times G / \Gamma \rtimes \Theta)$. The result now follows from Theorem 5.3.2.

Corollary 5.3.4. For $f \in C_{c}(G \rtimes \Theta)$, the bounded linear operator $R_{f}$ is a HilbertSchmidt operator.

Proof: By Corollary 5.3.3, the integral kernel of $R_{f}$ is in $L^{2}(G / \Gamma \rtimes \Theta \times G / \Gamma \rtimes \Theta)$. This implies, by Theorem 5.3.1, that $R_{f}$ is Hilbert-Schmidt.

\subsection{The Right Regular Representation of $G / \Gamma \rtimes \Theta$}

We will introduce the (right) regular representation $\bar{R}$ of $G / \Gamma \rtimes \Theta$, and the bounded linear operator $\bar{R}_{f}$, similar to what we did in $\S 4.1$. We will define the convolution $g * h$ of two functions in $L^{2}(H)$ for a locally compact group $H$. Using a theorem proved in [9], we will see that $\bar{R}_{f}$ is trace class if and only if $f \in L^{2}(G / \Gamma \rtimes \Theta) * L^{2}(G / \Gamma \rtimes \Theta)$. Similar to $\S 4.2$, we will prove that $R_{f}=\bar{R}_{f_{\Gamma}}$. Our goal will be to determine for which functions $f \in C_{c}(G \rtimes \Theta)$ we have that $R_{f}=\bar{R}_{f_{\Gamma}}$ is a trace class operator. We will prove that this operator is trace class whenever $f \in C_{c}(G \rtimes \Theta) * C_{c}(G \rtimes \Theta)$, and accomplish this by looking at $R_{f}$ for such a function $f$.

Let $\bar{R}: G / \Gamma \rtimes \Theta \rightarrow \mathcal{U}\left(L^{2}(G / \Gamma \rtimes \Theta)\right)$ be defined by

$$
(\bar{R}(x \Gamma, \tau) g)(y \Gamma, \theta)=g(y \theta(x) \Gamma, \theta \tau)
$$


for all $(x \Gamma, \tau),(y \Gamma, \theta) \in G / \Gamma \rtimes \Theta$ and $g \in L^{2}(G / \Gamma \rtimes \Theta)$. It is easy to check that $\bar{R}$ is well-defined. $\bar{R}$ is called the right regular representation of $G / \Gamma \rtimes \Theta$. That $\bar{R}$ is a unitary representation of $G / \Gamma \rtimes \Theta$ follows almost identically to the proof for the (right) regular representation of $G / \Gamma$ (Proposition 4.1.1).

Suppose $f \in C(G / \Gamma \rtimes \Theta)$. Note that $C(G / \Gamma \rtimes \Theta)$ is referring to the subspace of $L^{2}(G / \Gamma \rtimes \Theta)$ of functions equal $\mu_{G / \Gamma \rtimes \Theta}$-almost everywhere to a continuous function. Define $\bar{R}_{f}: L^{2}(G / \Gamma \rtimes \Theta) \rightarrow L^{2}(G / \Gamma \rtimes \Theta)$ by

$$
\begin{aligned}
\left(\bar{R}_{f} g\right)(y \Gamma, \theta) & =\int_{G / \Gamma \rtimes \Theta} f(x \Gamma, \tau)(\bar{R}(x \Gamma, \tau) g)(y \Gamma, \theta) d(x \Gamma, \tau) \\
& =\int_{G / \Gamma \rtimes \Theta} f(x \Gamma, \tau) g(y \theta(x) \Gamma, \theta \tau) d(x \Gamma, \tau) .
\end{aligned}
$$

for all $(y \Gamma, \theta) \in G / \Gamma \rtimes \Theta$ and $g \in L^{2}(G / \Gamma \rtimes \Theta)$. That $\bar{R}_{f}$ is a bounded linear operator follows almost identically to the proof of Proposition 4.1.2, and is omitted.

Is it always true that, for $f \in C(G / \Gamma \rtimes \Theta)$, the linear operator $\bar{R}_{f}$ is trace class? The answer is no. The set of functions $f$ that make $\bar{R}_{f}$ trace class needs some more specifications. For this we need to discuss the convolution of functions. Suppose $H$ is a locally compact group and $g, h \in L^{2}(H)$. The convolution, $g * h$, of $g$ and $h$ is the function on $H$ given by

$$
g * h(x)=\int_{H} g(y) h\left(y^{-1} x\right) d y
$$

for all $x \in H$, where integration is taken with respect to a Haar measure. If $H$ is unimodular, it is shown in ([7], §2.6) that the convolution is also given by

$$
g * h(x)=\int_{H} g\left(x y^{-1}\right) h(y) d y
$$

for all $x \in H$. As $G / \Gamma \rtimes \Theta$ is unimodular (since it is compact), we will be using (5.13) as our definition of the convolution. The next proposition follows from 
([7], Prop 2.40). Note that $G / \Gamma \rtimes \Theta$ being unimodular is an assumption necessary in the referenced proposition.

Proposition 5.4.1. Suppose that $g, h \in L^{2}(G / \Gamma \rtimes \Theta)$. Then $g * h \in C(G / \Gamma \rtimes \Theta)$.

Let $L^{2}(G / \Gamma \rtimes \Theta) * L^{2}(G / \Gamma \rtimes \Theta)$ denote the space of finite linear combinations of functions $f$ that can be written as $f=g * h$, where $g, h \in L^{2}(G / \Gamma \rtimes \Theta)$. In our case, should $f$ belong to $L^{2}(G / \Gamma \rtimes \Theta) * L^{2}(G / \Gamma \rtimes \Theta)$, Proposition 5.4.1 assures us that $f$ is continuous. Since we defined $\bar{R}_{f}$ only for continuous functions, this is an important result. It allows us to use the next theorem, which is proved with greater generality in $([9]$, Thm 34.16).

Theorem 5.4.2. Let $f \in C(G / \Gamma \rtimes \Theta)$. Then $\bar{R}_{f}$ is trace class if and only if $f \in$ $L^{2}(G / \Gamma \rtimes \Theta) * L^{2}(G / \Gamma \rtimes \Theta)$.

We now know the exact subset of continuous functions $f$ on $G / \Gamma \rtimes \Theta$ that make $\bar{R}_{f}$ a trace class operator.

Proposition 5.4.3. For $f \in C_{c}(G \rtimes \Theta)$, we have $R_{f}=\bar{R}_{f_{\Gamma}}$. 
Proof: Once again we compute a change of variable, seeking a connection between $R_{f}$ and $\bar{R}_{f_{\Gamma}}$. Suppose that $g \in L^{2}(G / \Gamma \rtimes \Theta)$ and $(y \Gamma, \theta) \in G / \Gamma \rtimes \Theta$. Then

$$
\begin{array}{rlr}
\left(\bar{R}_{f_{\Gamma}} g\right)(y \Gamma, \theta) & =\int_{G / \Gamma \rtimes \Theta} f_{\Gamma}(x \Gamma, \tau) g(y \theta(x) \Gamma, \theta \tau) d(x \Gamma, \tau) & \\
& =\sum_{\tau \in \Theta} \int_{G / \Gamma} f_{\Gamma}(x \Gamma, \tau) g(y \theta(x) \Gamma, \theta \tau) d(x \Gamma) & \\
& =\sum_{\beta \in \Theta} \int_{G / \Gamma} f_{\Gamma}\left(x \Gamma, \theta^{-1} \beta\right) g(y \theta(x) \Gamma, \beta) d(x \Gamma) & \\
& =\sum_{\beta \in \Theta} \int_{G / \Gamma} f_{\Gamma}\left(\theta^{-1}\left(y^{-1} z\right) \Gamma, \theta^{-1} \beta\right) g(z \Gamma, \beta) d(z \Gamma) & \\
& =\int_{G / \Gamma \rtimes \Theta}\left[\sum_{\gamma \in \Gamma} f\left(\theta^{-1}\left(y^{-1} z\right) \gamma, \theta^{-1} \beta\right)\right] g(z \Gamma, \beta) d(z \Gamma, \beta) &
\end{array}
$$

Since $\Gamma$ is stable under $\theta^{-1}$, we can replace $\gamma$ with $\theta^{-1}(\gamma)$.

$$
\begin{aligned}
& =\int_{G / \Gamma \rtimes \Theta}\left[\sum_{\gamma \in \Gamma} f\left(\theta^{-1}\left(y^{-1} \gamma z\right), \theta^{-1} \beta\right)\right] g(z \Gamma, \beta) d(z \Gamma, \beta) \\
& =\int_{G / \Gamma \rtimes \Theta} K_{f}(y \Gamma, \theta, z \Gamma, \beta) g(z \Gamma, \beta) d(z \Gamma, \beta) \\
& =\left(R_{f} g\right)(y \Gamma, \theta) .
\end{aligned}
$$

Therefore $R_{f}=\bar{R}_{f_{\Gamma}}$.

We have determined that the space of functions $f_{\Gamma}$ where $\bar{R}_{f_{\Gamma}}$ is trace class is $L^{2}(G / \Gamma \rtimes$ $\Theta) * L^{2}(G / \Gamma \rtimes \Theta)$. Can we get as strong of a result for $R_{f}$ ? Which functions $f \in C_{c}(G \rtimes \Theta)$ will make $R_{f}$ a trace class operator? If we consider the map from $C_{c}(G \rtimes \Theta)$ to $C(G / \Gamma \rtimes \Theta)$ given by the $\Gamma$-projection $f \mapsto f_{\Gamma}$, by Proposition 5.4.3 the desired set of functions will be the pre-image of the set $L^{2}(G / \Gamma \rtimes \Theta) * L^{2}(G / \Gamma \rtimes \Theta)$.

Let $C_{c}(G \rtimes \Theta) * C_{c}(G \rtimes \Theta)$ denote the space of finite linear combinations of functions $f$ which can be written as $f=g * h$, where $g, h \in C_{c}(G \rtimes \Theta)$. We now show that 
$C_{c}(G \rtimes \Theta) * C_{c}(G \rtimes \Theta)$ is a subset of the pre-image of $L^{2}(G / \Gamma \rtimes \Theta) * L^{2}(G / \Gamma \rtimes \Theta)$ with respect to the map $f \mapsto f_{\Gamma}$. In order to do so, we must understand the action of the $\Gamma$-projection on the convolution of two functions.

Proposition 5.4.4. Let $H$ be a locally compact group and $g, h \in C_{c}(H)$. Then $g * h \in C_{c}(H)$.

Proof: Since we have $C_{c}(H) \subseteq L^{2}(H)([10], \S 13)$, by Proposition 5.4.1 we know that $g * h$ is continuous. Let $A=\operatorname{supp}(g)$ and $B=\operatorname{supp}(h)$. By our assumptions $A$ and $B$ are compact. For $x, y \in H$, consider $g\left(x y^{-1}\right) h(y)$. We have $g\left(x y^{-1}\right) h(y)=0$ unless $y \in B$ and $x y^{-1} \in A$. If $x \notin A B$, then for every $y \in B, x y^{-1} \notin A$. Thus

$$
g * h(x)=\int_{H} g\left(x y^{-1}\right) h(y) d y=0
$$

Therefore $g * h$ vanishes outside of $A B$, so $\operatorname{supp}(g * h) \subseteq A B$. The set $A B$ is the continuous image of the compact set $A \times B$ under multiplication, and thus is compact ([23], §6.1). Since $\operatorname{supp}(g * h)$ is a closed subset of the compact set $A B$, we have that $\operatorname{supp}(g * h)$ is compact $([23], \S 6.1)$. This proves that $g * h \in C_{c}(H)$.

Proposition 5.4.4 shows that we are able to take the $\Gamma$-projection of the convolution of two functions in $C_{c}(G \rtimes \Theta)$. The following proposition shows exactly what that $\Gamma$-projection will be.

Proposition 5.4.5. Suppose that $g, h \in C_{c}(G \rtimes \Theta)$ and $f=g * h$. Then $f_{\Gamma}=g_{\Gamma} * h_{\Gamma}$. 
Proof: Let $(y \Gamma, \theta) \in G / \Gamma \rtimes \Theta$. Then

$$
\begin{aligned}
f_{\Gamma}(y \Gamma, \theta) & =\sum_{\gamma \in \Gamma} f(y \gamma, \theta) \\
& =\sum_{\gamma \in \Gamma} \int_{G \rtimes \Theta} g\left((y \gamma, \theta)\left(\tau^{-1}\left(x^{-1}\right), \tau^{-1}\right)\right) h(x, \tau) d(x, \tau) \\
& =\sum_{\gamma \in \Gamma} \int_{G \rtimes \Theta} g\left(y \gamma \theta\left(\tau^{-1}\left(x^{-1}\right)\right), \theta \tau^{-1}\right) h(x, \tau) d(x, \tau) \\
& =\sum_{\gamma \in \Gamma} \int_{G / \Gamma \rtimes \Theta} \sum_{\gamma_{1} \in \Gamma} g\left(y \gamma \theta\left(\tau^{-1}\left(\gamma_{1}^{-1} x^{-1}\right)\right), \theta \tau^{-1}\right) h\left(x \gamma_{1}, \tau\right) d(x \Gamma, \tau) \\
& =\sum_{\gamma \in \Gamma} \sum_{\gamma_{1} \in \Gamma} \int_{G / \Gamma \rtimes \Theta} g\left(y \gamma \theta\left(\tau^{-1}\left(\gamma_{1}^{-1}\right)\right) \theta\left(\tau^{-1}\left(x^{-1}\right)\right), \theta \tau^{-1}\right) h\left(x \gamma_{1}, \tau\right) d(x \Gamma, \tau) \\
& =\sum_{\gamma_{1} \in \Gamma} \int_{G / \Gamma \rtimes \Theta} \sum_{\gamma \in \Gamma} g\left(y \gamma \theta\left(\tau^{-1}\left(\gamma_{1}^{-1}\right)\right) \theta\left(\tau^{-1}\left(x^{-1}\right)\right), \theta \tau^{-1}\right) h\left(x \gamma_{1}, \tau\right) d(x \Gamma, \tau)
\end{aligned}
$$

Set $\gamma^{\prime}=\gamma \theta\left(\tau^{-1}\left(\gamma_{1}^{-1}\right)\right)$. Note that $\Gamma$ is invariant under this substitution.

$$
\begin{aligned}
& =\sum_{\gamma_{1} \in \Gamma} \int_{G / \Gamma \rtimes \Theta} \sum_{\gamma^{\prime} \in \Gamma} g\left(y \gamma^{\prime} \theta\left(\tau^{-1}\left(x^{-1}\right)\right), \theta \tau^{-1}\right) h\left(x \gamma_{1}, \tau\right) d(x \Gamma, \tau) \\
& =\sum_{\gamma_{1} \in \Gamma} \int_{G / \Gamma \rtimes \Theta} g_{\Gamma}\left(y \theta\left(\tau^{-1}\left(x^{-1}\right) \Gamma, \theta \tau^{-1}\right) h\left(x \gamma_{1}, \tau\right) d(x \Gamma, \tau)\right. \\
& =\int_{G / \Gamma \rtimes \Theta} g_{\Gamma}\left((y \Gamma, \theta)\left(\tau^{-1}\left(x^{-1}\right) \Gamma, \tau^{-1}\right)\right) \sum_{\gamma_{1} \in \Gamma} h\left(x \gamma_{1}, \tau\right) d(x \Gamma, \tau) \\
& =\int_{G / \Gamma \rtimes \Theta} g_{\Gamma}\left((y \Gamma, \theta)\left(\tau^{-1}\left(x^{-1}\right) \Gamma, \tau^{-1}\right)\right) h_{\Gamma}(x \Gamma, \tau) d(x \Gamma, \tau) \\
& =\left(g_{\Gamma} * h_{\Gamma}\right)(y \Gamma, \theta) .
\end{aligned}
$$

Therefore $f_{\Gamma}=g_{\Gamma} * h_{\Gamma}$.

Proposition 5.4.5 shows that when $f \in C_{c}(G \rtimes \Theta) * C_{c}(G \rtimes \Theta)$, the $\Gamma$-projection $f_{\Gamma} \in C(G / \Gamma \rtimes \Theta) * C(G / \Gamma \rtimes \Theta) \subseteq L^{2}(G / \Gamma \rtimes \Theta) * L^{2}(G / \Gamma \rtimes \Theta)$. Therefore, by Theorem 5.4.2, we have that $\bar{R}_{f_{\Gamma}}$ is trace class. Since $R_{f}=\bar{R}_{f_{\Gamma}}$ by Proposition 5.4.3, we conclude that $R_{f}$ is trace class. The next proposition shows another way we can prove that $R_{f}$ is trace class in this situation, and what $R_{f}$ will look like. 
Proposition 5.4.6. Suppose $f=f_{1} * f_{2}$, where $f_{1}, f_{2} \in C_{c}(G \rtimes \Theta)$. Then $R_{f}=$ $R_{f_{1}} R_{f_{2}}$.

Proof: Let $g \in L^{2}(G / \Gamma \rtimes \Theta)$ and $(y \Gamma, \theta) \in G / \Gamma \rtimes \Theta$. Then

$$
\begin{aligned}
\left(R_{f_{1}}\left(R_{f_{2}} g\right)\right)(y \Gamma, \theta) & =\int_{G \rtimes \Theta} f_{1}(x, \tau)\left(R(x, \tau)\left(R_{f_{2}} g\right)\right)(y \Gamma, \theta) d(x, \tau) \\
& \left.=\int_{G \rtimes \Theta} f_{1}(x, \tau)\left(R_{f_{2}} g\right)\right)(y \theta(x) \Gamma, \theta \tau) d(x, \tau) \\
& =\int_{G \rtimes \Theta} f_{1}(x, \tau) \int_{G \rtimes \Theta} f_{2}(z, \sigma)(R(z, \sigma) g)((y \Gamma, \theta)(x \Gamma, \tau)) d(z, \sigma) d(x, \tau) \\
& =\int_{G \rtimes \Theta} \int_{G \rtimes \Theta} f_{1}(x, \tau) f_{2}(z, \sigma) g((y \Gamma, \theta)(x \tau(z) \Gamma, \tau \sigma)) d(x, \tau) d(z, \sigma) \\
& \text { Set }\left(x^{\prime}, \tau^{\prime}\right)=(x, \tau)(z, \sigma) . \text { Note } d(x, \tau)=d\left(x^{\prime}, \tau^{\prime}\right) . \\
& =\int_{G \rtimes \Theta} \int_{G \rtimes \Theta} f_{1}\left(\left(x^{\prime}, \tau^{\prime}\right)(z, \sigma)^{-1}\right) f_{2}(z, \sigma) g\left((y \Gamma, \theta)\left(x^{\prime} \Gamma, \tau^{\prime}\right) d\left(x^{\prime}, \tau^{\prime}\right) d(z, \sigma)\right. \\
& \operatorname{Rename} x=x^{\prime}, \tau=\tau^{\prime} . \\
& =\int_{G \rtimes \Theta} \int_{G \rtimes \Theta} f_{1}\left((x, \tau)(z, \sigma)^{-1}\right) f_{2}(z, \sigma) d(z, \sigma) g((y \Gamma, \theta)(x \Gamma, \tau)) d(x, \tau) \\
& =\int_{G \rtimes \Theta}\left(f_{1} * f_{2}\right)(x, \tau)(R(x, \tau) g)(y \Gamma, \theta) d(x, \tau) \\
& =\left(R_{f_{1} * f_{2}} g\right)(y \Gamma, \theta) .
\end{aligned}
$$

Therefore $R_{f}=R_{f_{1}} R_{f_{2}}$.

In $\S 5.3$, we proved that $R_{f}$ is Hilbert-Schmidt when $f \in C_{c}(G \rtimes \Theta)$. Thus, Proposition 5.4.6 shows that when $f \in C_{c}(G \rtimes \Theta) * C_{c}(G \rtimes \Theta), R_{f}$ is the composition of two Hilbert-Schmidt operators. As mentioned in $\S 5.3$, this proves that $R_{f}$ is a trace class operator $([21], \S 6.6)$. As we have just discussed, the previous two propositions have proved the next theorem. 
Theorem 5.4.7. If $f \in C_{c}(G \rtimes \Theta) * C_{c}(G \rtimes \Theta)$, then $R_{f}=\bar{R}_{f_{\Gamma}}$ is a trace class operator.

We have found a subset of $C_{c}(G \rtimes \Theta)$ where $R_{f}$ is trace class. What still remains an open question is whether we can find a more specific result. Is the space of functions where $R_{f}$ is trace class exactly $C_{c}(G \rtimes \Theta) * C_{c}(G \rtimes \Theta)$, or are there more functions? Consider the map $P: C_{c}(G \rtimes \Theta) \rightarrow C(G / \Gamma \rtimes \Theta)$ given by the $\Gamma$-projection. It was mentioned in $\S 5.2$ that $P$ is surjective, and it maps $C_{c}(G \rtimes \Theta) * C_{c}(G \rtimes \Theta)$ into $C(G / \Gamma \rtimes \Theta) * C(G / \Gamma \rtimes \Theta)$ by Proposition 5.4.5. Therefore, if $C(G / \Gamma \rtimes \Theta) * C(G / \Gamma \rtimes \Theta) \neq$ $L^{2}(G / \Gamma \rtimes \Theta) * L^{2}(G / \Gamma \rtimes \Theta)$, we actually can find some functions $f \in C_{c}(G \rtimes \Theta)$ such that $f \notin C_{c}(G \rtimes \Theta) * C_{c}(G \rtimes \Theta)$ but $R_{f}=\bar{R}_{f_{\Gamma}}$ is trace class.

There are some topological groups where we in fact do have $C_{c}(G \rtimes \Theta) * C_{c}(G \rtimes \Theta)=$ $C_{c}(G \rtimes \Theta)$, as shown in the following proposition. In this special case, $R_{f}=\bar{R}_{f_{\mathrm{\Gamma}}}$ is trace class for every $f \in C_{c}(G \rtimes \Theta)$.

Corollary 5.4.8. Suppose that $G \rtimes \Theta$ is a discrete group. Then $C_{c}(G \rtimes \Theta) * C_{c}(G \rtimes \Theta)=C_{c}(G \rtimes \Theta)$.

Proof: Proposition 5.4.4 proved that $C_{c}(G \rtimes \Theta) * C_{c}(G \rtimes \Theta) \subseteq C_{c}(G \rtimes \Theta)$. Suppose $f \in C_{c}(G \rtimes \Theta)$. Let $h: G \rtimes \Theta \rightarrow \mathbb{C}$ be defined by

$$
h(y \Gamma, \theta)=\left\{\begin{array}{ll}
1 & \text { if }(y \Gamma, \theta)=\left(e \Gamma, e_{\Theta}\right) \\
0 & \text { otherwise }
\end{array} .\right.
$$

The function $h$ is continuous, since all function on a discrete space are clearly continuous, and has $\operatorname{supp}(f)=\left\{\left(e \Gamma, e_{\Theta}\right)\right\}$, which is compact. Thus $h \in C_{c}(G \rtimes \Theta)$. As $h$ 
has a value of 0 everywhere except for at the identity of $G \rtimes \Theta$, we have that

$$
\begin{aligned}
(f * h)(y, \theta) & =\int_{G \rtimes \Theta} f\left((y, \theta)\left(\tau^{-1}\left(x^{-1}\right), \tau^{-1}\right)\right) h(x, \tau) d(x, \tau) \\
& =\int_{\{e\} \times\left\{e_{\Theta}\right\}} f(y, \theta) d \mu \\
& =f(y, \theta)
\end{aligned}
$$

where we are integrating with respect to the counting measure $\mu$ on $G \rtimes \Theta$. Therefore $f=f * h$, proving that $C_{c}(G \rtimes \Theta) \subseteq C_{c}(G \rtimes \Theta) * C_{c}(G \rtimes \Theta)$.

\subsection{Computing the Trace of $R_{f}$}

Now that we have a subspace of functions $f \in C_{c}(G \rtimes \Theta)$ for which $R_{f}$ is trace class, we can focus on actually computing what this trace is. We will use a theorem of Duflo proved in $([4], \S 5.3)$ to compute the trace value.

As Duflo works in great generality, we must check that all assumptions of his theorem are satisfied. He assumes a locally compact group which is the countable union of compact spaces. Since $G / \Gamma \rtimes \Theta$ is compact, the assumption is clearly satisfied. He assumes a Radon measure. The measure $\mu_{G / \Gamma \rtimes \Theta}$ satisfies this, since Haar measures are Radon measures ( $\$ 2.1)$. He assumes that the continuous functions on the group are dense in the space of $L^{2}$-functions on the group. This holds in our case, as Lemma 2.2.5 proved that $C(G / \Gamma \rtimes \Theta)$ is dense in $L^{2}(G / \Gamma \rtimes \Theta)$. He also assumes the existence of an integral kernel that is continuous and bounded. Our integral kernel $K_{f}$ satisfies this, as proved by Theorem 5.3.2 and Corollary 5.3.3. Finally, he assumes that the support of the measure is the entire space, where the support of a measure is the smallest closed subset of the measure space which has a compliment of mea- 
sure zero ([3], §7.4)). The closed set $G / \Gamma \rtimes \Theta$ satisfies this assumption, as proved by Lemma 2.1.1, which implies the only open set of measure zero in $G / \Gamma \rtimes \Theta$ is the empty set.

Therefore, all of Duflo's assumptions for Proposition 3.3.1 in [4] are satisfied and we get the following particular case.

Theorem 5.5.1. Suppose that $R_{f}$ is trace class for some $f \in C_{c}(G \rtimes \Theta)$. Then the function $(y \Gamma, \theta) \mapsto K_{f}(y \Gamma, \theta, y \Gamma, \theta)$ is integrable and the trace of $R_{f}$ is given by

$$
\operatorname{tr}\left(R_{f}\right)=\int_{G / \Gamma \rtimes \Theta} K_{f}(y \Gamma, \theta, y \Gamma, \theta) d(y \Gamma, \theta) .
$$

Note that the values on the diagonal of $K_{f}$ are

$$
\begin{aligned}
K_{f}(y \Gamma, \theta, y \Gamma, \theta) & =f_{\Gamma}\left(\theta^{-1}\left(y^{-1} y\right) \Gamma, \theta^{-1} \theta\right) \\
& =f_{\Gamma}\left(\Gamma, e_{\Theta}\right) .
\end{aligned}
$$

Therefore, we get the following result.

Corollary 5.5.2. If $R_{f}$ is trace class for some $f \in C_{c}(G \rtimes \Theta)$, then

$$
\operatorname{tr}\left(R_{f}\right)=\operatorname{tr}\left(\bar{R}_{f_{\Gamma}}\right)=|\Theta| f_{\Gamma}\left(e \Gamma, e_{\Theta}\right)=|\Theta| \sum_{\gamma \in \Gamma} f\left(\gamma, e_{\Theta}\right)
$$

In particular, this holds when $f \in C_{c}(G \rtimes \Theta) * C_{c}(G \rtimes \Theta)$. 
Proof: Suppose $f \in C_{c}(G \rtimes \Theta)$ such that $R_{f}$ is trace class. By Theorem 5.5.1, we know that

$$
\begin{aligned}
\operatorname{tr}\left(R_{f}\right) & =\int_{G / \Gamma \rtimes \Theta} K_{f}(y \Gamma, \theta, y \Gamma, \theta) d(y \Gamma, \theta) \\
& =\int_{G / \Gamma \rtimes \Theta} f_{\Gamma}\left(\theta^{-1}\left(y^{-1} y\right) \Gamma, \theta^{-1} \theta\right) d(y \Gamma, \theta) \\
& =\int_{G / \Gamma \rtimes \Theta} f_{\Gamma}\left(\Gamma, e_{\Theta}\right) d(y \Gamma, \theta) \\
& =\sum_{\theta \in \Theta} \int_{G / \Gamma} f_{\Gamma}\left(\Gamma, e_{\Theta}\right) d(y \Gamma, \theta) \\
& =\sum_{\theta \in \Theta} f_{\Gamma}\left(\Gamma, e_{\Theta}\right) \mu_{G / \Gamma}(G / \Gamma \rtimes \Theta) \\
& =|\Theta| f_{\Gamma}\left(\Gamma, e_{\Theta}\right) \\
& =|\Theta| \sum_{\gamma \in \Gamma} f\left(\gamma, e_{\Theta}\right) .
\end{aligned}
$$

The second assertion follows from Theorem 5.4.7.

We have made the conclusion that the trace of $R_{f}$, should $R_{f}$ be trace class, only depends on the values $f_{\Gamma}$ attains at the identity $e_{\Theta}$ of $\Theta$, or in other words the values of $f$ on all of $\Gamma$.

\section{Example:}

Consider $G=\mathbb{Z}$ as a group under addition with the discrete topology. Let $\Gamma=3 \mathbb{Z}$, so that $G / \Gamma=\mathbb{Z} / 3 \mathbb{Z} \cong \mathbb{Z}_{3}$, and $\mu_{\mathbb{Z}}$ be the counting measure on $\mathbb{Z}$. Let $\Theta=\operatorname{Aut}(\mathbb{Z})=$ $\{i d, \theta\}$, where $i d$ is the identity automorphism and $\theta$ is the automorphism $\theta(m)=-m$. Note that $3 \mathbb{Z}$ is stable under $\Theta$.

Let $f: \mathbb{Z} \rtimes \Theta$ be defined by

$$
f(m, \tau)=\left\{\begin{array}{ll}
m & \text { if } \tau=i d \text { and } 0 \leqslant m \leqslant 5 \\
0 & \text { otherwise }
\end{array} .\right.
$$


Since $\mathbb{Z} \rtimes \Theta$ is a discrete group and the support of $f$ is the compact set $\{(0, i d),(1, i d),(2, i d),(3, i d)$, $(4, i d),(5, i d)\}$, we have that $f \in C_{c}(\mathbb{Z} \rtimes \Theta)$. The bounded linear operator $R_{f}$ is given by

$$
\begin{aligned}
\left(R_{f} g\right)(m, \tau) & =\int_{\mathbb{Z} \times \Theta} f(k, \sigma)(R(k, \sigma) g)(\bar{m}, \tau) d(k, \sigma) \\
& =\int_{\mathbb{Z}} f(k, i d) g(\overline{m \tau(k)}, \tau) d k+\int_{\mathbb{Z}} f(k, \theta) g(\overline{m \tau(k)}, \tau \theta) d k \\
& =\sum_{k=0}^{5} k g(\overline{m \tau(k)}, \tau)
\end{aligned}
$$

for all $g \in L^{2}\left(\mathbb{Z}_{3} \rtimes \Theta\right)$. If we look at the $\Gamma$-projection of $f$, we see that, for $0 \leqslant m \leqslant 2$

$$
\begin{gathered}
f_{\Gamma}(\bar{m}, i d)=f(m, i d)+f(m+3, i d)=2 m+3 . \\
f_{\Gamma}(\bar{m}, \theta)=0 .
\end{gathered}
$$

As $\mathbb{Z} \rtimes \Theta$ is discrete, by Theorem 5.4.7 and Proposition 5.4.8 we know that $R_{f}=\bar{R}_{f_{\Gamma}}$ is a trace class operator. Using Corollary 5.5.2, we have that

$$
\begin{aligned}
\operatorname{tr}\left(R_{f}\right) & =\operatorname{tr}\left(\bar{R}_{f_{\Gamma}}\right) \\
& =|\Theta| f_{\Gamma}(\overline{0}, i d) \\
& =6 .
\end{aligned}
$$




\section{Chapter 6}

\section{Irreducible Representations of $G / \Gamma \rtimes \Theta$}

We continue to work under the assumption that $G$ is a second countable LCA group with a discrete subgroup $\Gamma$ such that $G / \Gamma$ is compact, and that $\Theta$ is a finite subgroup of $\operatorname{Aut}(G)$. We will classify all irreducible unitary representations of $G / \Gamma \rtimes \Theta$ and determine their trace values. The process for finding these irreducible representations follows the presentation given in $([19], \S 8.2)$ for the case of a finite group. The proof of Mackey's Irreducibility Criterion has been excluded. It is proved in ([12], Thm 5.20) in the case of a finite group, but it is mentioned that in actuality the proof only depends on the assumption of complete reducibility, which holds for compact groups ([7], §5.1). Therefore Mackey's Irreducibility Criterion holds true in the case of a compact group.

\subsection{A Group Action of $\Theta$ on $\widehat{G / \Gamma}$}

We will let the group $\Theta$ act on the continuous characters $\widehat{G / \Gamma}$. Using a complete 
set of orbit representatives $\left\{\varphi_{j}: j \in J\right\}$, we will create irreducible representations of $G / \Gamma \rtimes \Theta_{\varphi_{\jmath}}$, where $\Theta_{\varphi_{j}}$ is the stabilizer of $\varphi_{\jmath}$. These irreducible representations will be identified with the tensor products of $\varphi_{j}$ with an irreducible unitary representations $\rho$ of the finite group $\Theta_{\varphi_{3}}$. More precisely, they will be given by $\left(\varphi_{j} \otimes \rho\right)(y \Gamma, \theta)=\varphi_{j}(y \Gamma) \rho(\theta)$ for every $(y \Gamma, \theta) \in G / \Gamma \rtimes \Theta$.

We define a (left) group action of the group $\Theta$ on the set $\widehat{G / \Gamma}$ by

$$
(\theta \cdot \varphi)(x \Gamma)=\varphi\left(\theta^{-1}(x) \Gamma\right)
$$

for all $x \Gamma \in G / \Gamma, \theta \in \Theta$ and $\varphi \in \widehat{G / \Gamma}$. Let $\left\{\Theta \cdot \varphi_{j}: j \in J\right\}$ denote the set of distinct orbits $\{\Theta \cdot \varphi: \varphi \in \widehat{G / \Gamma}\}$, where $J$ is the appropriate index set, and let $\Theta_{\varphi_{j}}=\left\{\theta \in \Theta: \theta \cdot \varphi_{j}=\varphi_{j}\right\}$ be the stabilizer subgroup of $\varphi_{j}$. Consider the subgroup $G / \Gamma \rtimes \Theta_{\varphi_{j}}=\left\{(x \Gamma, \theta): \quad x \Gamma \in G / \Gamma, \quad \theta \in \Theta_{\varphi_{\jmath}}\right\}$ of $G / \Gamma \rtimes \Theta$. We will first consider irreducible representations of $G / \Gamma \rtimes \Theta_{\varphi_{3}}$ and later induce them to create irreducible representations of $G / \Gamma \rtimes \Theta$.

Let $\rho: \Theta_{\varphi_{j}} \rightarrow \mathcal{U}\left(V_{j, \rho}\right)$ be an irreducible unitary representation of the finite group $\Theta_{\varphi_{\jmath}}$. Since $\Theta_{\varphi}$ is finite, $\rho$ is a finite-dimensional representation ([7], §5.1), meaning the complex vector space $V_{j, \rho}$ is finite-dimensional. Define $\varphi_{j} \otimes \rho: G / \Gamma \rtimes \Theta_{\varphi_{j}} \rightarrow \mathcal{U}\left(V_{j, \rho}\right)$ by

$$
\left(\varphi_{j} \otimes \rho\right)(y \Gamma, \theta)=\varphi_{\jmath}(y \Gamma) \rho(\theta)
$$

for every $(y \Gamma, \theta) \in G / \Gamma \rtimes \Theta$.

Proposition 6.1.1. $\varphi_{j} \otimes \rho$ is an irreducible unitary representation of $G / \Gamma \rtimes \Theta_{\varphi_{j}}$.

Proof: Let $(y \Gamma, \theta),(x \Gamma, \tau) \in G / \Gamma \rtimes \Theta_{\varphi_{\jmath}}$. Since $\theta^{-1} \in \Theta_{\varphi_{\jmath}}$, we have $\theta^{-1} \cdot \varphi_{\jmath}=\varphi_{\jmath}$. 
Therefore

$$
\begin{aligned}
\left(\left(\varphi_{\jmath} \otimes \rho\right)(y \Gamma, \theta)\right)\left(\left(\varphi_{\jmath} \otimes \rho\right)(x \Gamma, \tau)\right) & =\varphi_{\jmath}(y \Gamma) \rho(\theta) \varphi_{\jmath}(x \Gamma) \rho(\tau) \\
& =\varphi_{\jmath}(y \Gamma)\left(\theta^{-1} \cdot \varphi_{\jmath}\right)(x \Gamma) \rho(\theta) \rho(\tau) \\
& =\varphi_{\jmath}(y \Gamma) \varphi_{\jmath}(\theta(x) \Gamma) \rho(\theta \tau) \\
& =\left(\varphi_{\jmath} \otimes \rho\right)(y \theta(x) \Gamma, \theta \tau)
\end{aligned}
$$

Thus, $\varphi_{\jmath} \otimes \rho$ preserves the group law. We now must check that $\varphi_{\jmath} \otimes \rho$ is continuous. Since we have equipped $G L\left(V_{\jmath, \rho}\right)$ with the strong operator topology, as mentioned in $\S 4.1$, it suffices to show that as $(x \Gamma, \theta) \rightarrow\left(e \Gamma, e_{\Theta}\right)$ in $G / \Gamma \rtimes \Theta_{\varphi_{\jmath}}$, then for all $v \in V_{\jmath, \rho}$ we have $\varphi_{\jmath} \otimes \rho(x \Gamma, \theta) v \rightarrow v$ in $V_{\jmath, \rho}$. Since $\Theta$ is discrete, eventually $(x \Gamma, \theta)$ becomes $\left(x \Gamma, e_{\Theta}\right)$ as it approaches $\left(e \Gamma, e_{\Theta}\right)$. Therefore, we only need to show that $\left(\varphi_{\jmath} \otimes \rho\right)\left(x \Gamma, e_{\Theta}\right) v \rightarrow v$ in $V_{\jmath, \rho}$. We have that

$$
\left(\varphi_{\jmath} \otimes \rho\right)\left(x \Gamma, e_{\Theta}\right) v=\varphi_{\jmath}(x \Gamma) \rho\left(e_{\Theta}\right) v=\varphi_{\jmath}(x \Gamma) v \rightarrow \varphi_{\jmath}(e \Gamma) v=v
$$

as $\varphi_{\jmath}(x \Gamma) \rightarrow \varphi_{\jmath}(e \Gamma)$ follows from the continuity of $\varphi_{\jmath}$. This proves that $\varphi_{\jmath} \otimes \rho$ is continuous. That $\varphi_{j} \otimes \rho$ is unitary follows easily from $\rho$ being unitary and the image of $\varphi_{\jmath}$ being in the unit circle. Thus, $\varphi_{\jmath} \otimes \rho$ is indeed a unitary representation of $G / \Gamma \rtimes \Theta_{\varphi_{3}}$.

All that remains to be shown is that it is irreducible. Let $W$ be an invariant proper subspace of $V_{\jmath, \rho}$ with respect to the representation $\varphi_{\jmath} \otimes \rho$ of $G / \Gamma \rtimes \Theta_{\varphi_{j}}$. This means that for all $w \in W$ and $(x \Gamma, \theta) \in G / \Gamma \rtimes \Theta_{\varphi_{\jmath}}$, we have $\varphi_{\jmath} \otimes \rho(x \Gamma, \theta) w=\varphi_{\jmath}(x \Gamma) \rho(\theta) w \in W$. In particular, for every $\theta \in \Theta_{\varphi_{\jmath}}, \varphi_{\jmath} \otimes \rho(\Gamma, \theta) w=\rho(\theta) w \in W$. So $W$ is an invariant proper subspace of $V_{\jmath, \rho}$ with respect to the representation $\rho$ of $\Theta_{\varphi_{j}}$. Since $\rho$ is irreducible, $W=\{0\}$. Therefore the only invariant subspaces of $V_{\jmath, \rho}$ with respect to the representation $\varphi_{\jmath} \otimes \rho$ of $G / \Gamma \rtimes \Theta_{\varphi_{j}}$ are $W$ and $\{0\}$, proving that $\varphi_{\jmath} \otimes \rho$ is irreducible. 


\subsection{Induced Representations and Mackey's Irre- ducibility Criterion}

We will describe induced spaces of representations. We will state Mackey's Irreducibility Criterion, and use it to prove that the induced representation of $\varphi_{\jmath} \otimes \rho$ from $G / \Gamma \rtimes \Theta_{\varphi,}$ to $G / \Gamma \rtimes \Theta$ is irreducible.

Consider a compact group $H$. Let $V$ be a finite-dimensional non-zero complex Hilbert space with inner product $\langle\cdot, \cdot\rangle$. Set $\|\cdot\|$ to be the norm corresponding to this inner product. Let $L^{2}(H, V)$ denote the space of functions (under equivalence of almost everywhere equality) such that

$$
\int_{H}\|f(x)\|^{2} d x<\infty
$$

where integration is taken with respect to a fixed Haar measure $\mu_{H}$. The space $L^{2}(H, V)$ forms a Hilbert space with respect to the $L^{2}$ inner product given by

$$
\left\langle f_{1}, f_{2}\right\rangle_{2}=\int_{H}\left\langle f_{1}(x), f_{2}(x)\right\rangle d x
$$

for every $f_{1}, f_{2} \in L^{2}(H, V)$. The $L^{2}$-norm on $L^{2}(H, V)$ is given by

$$
\|f\|_{2}=\sqrt{\langle f, f\rangle_{2}}
$$

for every $f \in L^{2}(H, V)$.

Let $K$ be a finite index subgroup of $H$ and $\omega: K \rightarrow G L(V)$ be a representation of $K$. Define $W_{\omega}^{H}=\left\{f \in L^{2}(H, V): f(k x)=\omega(k) f(x) \forall k \in K, x \in H\right\}$. The set $W_{\omega}^{H}$ forms a subspace of the Hilbert space $L^{2}(H, V)$, and we call it the induced space 
with respect to $\omega$. It can be shown that the induced space is finite-dimensional, which we will explicitly show for a particular case in $\S 6.3$. It is mentioned in $([7], \S 6.0)$ that if the assumption of finite index subgroup were removed, we could no longer conclude that the induced space is finite-dimensional. In fact, we could conclude in that case that the induced space would be infinite-dimensional.

The induced representation $\operatorname{Ind}_{K}^{H} \omega: H \rightarrow \mathcal{U}\left(W_{\omega}^{H}\right)$ of $\omega$ from $K$ to $H$ is defined by

$$
\left(\left(\operatorname{Ind} d_{K}^{H} \omega\right)(h) f\right)(x)=f(x h)
$$

for all $h, x \in H$ and $f \in W_{\omega}^{H}$. We first check that the image of $I n d_{K}^{H} \omega$ is contained in $\mathcal{U}\left(W_{\omega}^{H}\right)$. Suppose $h \in H$ and $f \in W_{\omega}^{H}$. Then

$$
\begin{aligned}
\left\|\left(\operatorname{Ind}_{K}^{H} \omega\right)(h) f\right\|_{2}^{2} & =\int_{H}\left\|\left(\left(\operatorname{Ind} d_{K}^{H} \omega\right)(h) f\right)(x)\right\|^{2} d x \\
& =\int_{H}\|f(x h)\|^{2} d x \\
& =\int_{H}\|f(x)\|^{2} d x \\
& =\|f\|_{2}^{2} \\
& <\infty .
\end{aligned}
$$

Thus, $\left(\operatorname{Ind}_{K}^{H} \omega\right)(h) f \in L^{2}(H, V)$. If $k \in K$ and $x \in H$, then

$$
\begin{aligned}
\left(\left(\operatorname{Ind}_{K}^{H} \omega\right)(h) f\right)(k x) & =f(k x h) \\
& =\omega(k) f(x h) \\
& =\omega(k)\left(\left(\operatorname{Ind}_{K}^{H} \omega\right)(h) f\right)(x) .
\end{aligned}
$$

This proves that indeed $\left(\operatorname{Ind}_{K}^{H} \omega\right)(h) f \in W_{\omega}^{H}$. We showed above that $\left\|\left(\operatorname{Ind}_{K}^{H} \omega\right)(h) f\right\|_{2}=$ $\|f\|_{2}$, which proves that $\left(\operatorname{Ind}_{K}^{H} \omega\right)(h) \in \mathcal{U}\left(W_{\omega}^{H}\right)$.

Proposition 6.2.1. Ind $d_{K}^{H} \omega$ is a unttary representation of $H$. 
Proof: We need to show the continuity of $\operatorname{In} d_{K}^{H} \omega$ in the strong operator topology. Suppose $h \rightarrow e$ is a convergent net in $H$ and $f \in W_{\omega}^{H}$. Following a similar augment to the one in $\S 2.2$ which proved that $C(H)$ is dense $L^{2}(H)$, it can be shown that the space of continuous functions $g: H \rightarrow V$, which we will denote by $C(H, V)$, is dense in $L^{2}(H, V)$.

Let $\epsilon>0$. Suppose $g \in C(H, V)$ is such that $\|f-g\|_{2}<\frac{\epsilon}{3}$. As $g$ is a continuous function on a compact group, we know that $g$ is uniformly continuous ([23], §9.2). Therefore we can choose a neighbourhood $U$ of $e$ such that for every $x \in H$ and every $h$ in our converging net that is residually in $U$, we have $\|g(x h)-g(x)\|<\frac{\epsilon}{3 \sqrt{\mu_{H}(H)}}$. For such $h$ we have that

$$
\begin{aligned}
\left\|I n d_{K}^{H} \omega(h) g-g\right\|_{2}^{2} & =\int_{H}\left\|\operatorname{Ind} d_{K}^{H} \omega(h) g(x)-g(x)\right\|^{2} d x \\
& =\int_{H}\|g(x h)-g(x)\|^{2} d x \\
& <\int_{H} \frac{\epsilon^{2}}{9 \mu_{H}(H)} d x \\
& =\frac{\epsilon^{2}}{9} .
\end{aligned}
$$

Therefore, for every such $h$ in our converging net that is residually in $U$, we have that

$$
\begin{aligned}
\left\|I n d_{K}^{H} \omega(h) f-f\right\|_{2} & \leqslant\left\|I n d_{K}^{H} \omega(h) f-\operatorname{In} d_{K}^{H} \omega(h) g\right\|_{2}+\left\|\operatorname{In} d_{K}^{H} \omega(h) g-g\right\|_{2}+\|g-f\|_{2} \\
& <\frac{\epsilon}{3}+\frac{\epsilon}{3}+\frac{\epsilon}{3}
\end{aligned}
$$

This proves that for $h \rightarrow e$ in $H$, we have $\left\|\operatorname{Ind} d_{K}^{H} \omega(h) f-f\right\|_{2} \rightarrow 0$. Thus $\operatorname{Ind} d_{K}^{H} \omega$ is continuous. 
Let $h_{1}, h_{2}, x \in H$ and $f \in W_{\omega}^{H}$. Then

$$
\begin{aligned}
\left(\operatorname{Ind}_{K}^{H} \omega\left(h_{1}\right)\left(\operatorname{Ind}_{K}^{H} \omega\left(h_{2}\right) f\right)\right)(x) & =\left(\operatorname{Ind}_{K}^{H} \omega\left(h_{2}\right) f\right)\left(x h_{1}\right) \\
& =f\left(x h_{1} h_{2}\right) \\
& =\left(\operatorname{Ind}_{K}^{H} \omega\left(h_{1} h_{2}\right) f\right)(x)
\end{aligned}
$$

Thus $\operatorname{Ind} d_{K}^{H} \omega\left(h_{1} h_{2}\right)=\operatorname{In} d_{K}^{H} \omega\left(h_{1}\right) \operatorname{In} d_{K}^{H} \omega\left(h_{2}\right)$, proving that $\operatorname{Ind} d_{K}^{H} \omega$ is a homomorphism. That $I n d_{K}^{H} \omega$ is unitary has already been shown. Therefore we have proven that $I n d_{K}^{H} \omega$ is a unitary representation.

Now let $\varrho: H \rightarrow G L(V)$ be a representation of $H$, where $V$ is a non-zero complex vector space. Then the restriction $\operatorname{Res}_{K}^{H} \varrho: K \rightarrow G L(V)$ of $\varrho$ to $K$ is defined by

$$
\operatorname{Res}_{K}^{H} \varrho(k)=\varrho(k)
$$

for all $k \in K$. Obviously, $\operatorname{Res}_{K}^{H} \varrho$ will be a representation of $K$, and unitary when $\varrho$ is unitary.

In order to state Mackey's Irreducibility Criterion, we need to to define some more notation. Let $H$ be a group with a subgroup $K$ and let $\omega: K \rightarrow A u t(V)$ be a representation of $K$. For $s \in H$, define $\omega^{s}: s K s^{-1} \rightarrow A u t(V)$ by $\omega^{s}(x)=\omega\left(s^{-1} x s\right)$ for all $x \in s K s^{-1}$. It can be shown that $\omega^{s}$ is a representation of the group $s K s^{-1}$. For any representation $\varrho: H \rightarrow A u t(W)$ of a group $H$, an invariant subspace of $W$ under $\varrho$ is a vector subspace $W^{\prime}$ of $W$ such that for every $h \in H$ and for all $w \in W^{\prime}$, $\varrho(h) w \in W^{\prime}$. A subrepresentation $\varrho^{\prime}$ of $\varrho$ is a representation $\varrho^{\prime}: H \rightarrow \operatorname{Aut}\left(W^{\prime}\right)$, where $W^{\prime}$ is an invariant subspace of $W$ under $\varrho$, such that $\varrho(h) w=\varrho^{\prime}(h) w$ for every $h \in H$ and $w \in W^{\prime}$. We will typically denote the subrepresentation by $\varrho^{\prime}=\left.\varrho\right|_{W^{\prime}}$. Two finite-dimensional representations of a group are called disjoint if none of their 
subrepresentations are equivalent. The following theorem is proved in ([12], Thm $5.20)$.

Theorem 6.2.2 (Mackey's Irreducibility Criterion). Let $H$ be a compact group, $K \leqslant H$ be a finite index subgroup, and $\omega: K \rightarrow G L(V)$ be a representation of $K$. Then Ind ${ }_{K}^{H} \omega$ is irreducible if and only if $\omega$ is irreducible and the representations $\operatorname{Res}_{K \cap s K s^{-1}}^{K} \omega$ and $\operatorname{Res}_{K n s K s^{-1}}^{s K s^{-1}} \omega^{s}$ are disjoint for all $s \in H$ with $s \notin K$.

Recall from $\S 6.1$ the representation $\varphi_{j} \otimes \rho$ of $G / \Gamma \rtimes \Theta_{\varphi_{j}}$. Consider the induced representation of $\varphi_{\jmath} \otimes \rho$ from $G / \Gamma \rtimes \Theta_{\varphi_{\jmath}}$ to $G / \Gamma \rtimes \Theta$, denoted by

$$
\psi_{3, \rho}=\operatorname{Ind} d_{G / \Gamma \times \Theta_{\varphi_{j}}^{G / \Gamma \times \Theta}\left(\varphi_{\jmath} \otimes \rho\right)}
$$

Note that $G / \Gamma \rtimes \Theta_{\varphi}$ is a finite index subgroup of $G / \Gamma \rtimes \Theta$ of index $\left|\Theta: \Theta_{\varphi_{\jmath}}\right|$.

Theorem 6.2.3. The induced representation $\psi_{\jmath, \rho}$ is irreducible for all $j \in J$ and irreducible representations $\rho$ of $\Theta_{\varphi_{j}}$.

Proof: To simplify notation, let $K=G / \Gamma \rtimes \Theta_{\varphi_{3}}$ and $K_{s}=s\left(G / \Gamma \rtimes \Theta_{\varphi_{\jmath}}\right) s^{-1}$, where $s \in G / \Gamma \rtimes \Theta$.

Let $s=(z \Gamma, \tau)$ be such that $s \notin G / \Gamma \rtimes \Theta_{\varphi_{3}}$. We have that

$$
\begin{aligned}
K \cap K_{s} & =\left(G / \Gamma \rtimes \Theta_{\varphi_{\jmath}}\right) \cap\left(s\left(G / \Gamma \rtimes \Theta_{\varphi_{\jmath}}\right) s^{-1}\right) \\
& =\left(G / \Gamma \rtimes \Theta_{\varphi_{\jmath}}\right) \cap\left(G / \Gamma \rtimes \tau\left(\Theta_{\varphi_{\jmath}}\right) \tau^{-1}\right) \\
& =G / \Gamma \rtimes\left(\Theta_{\varphi_{\jmath}} \cap \tau\left(\Theta_{\varphi_{\jmath}}\right) \tau^{-1}\right) .
\end{aligned}
$$

We need to show that $\operatorname{Res}_{K \cap K_{s}}^{K} \varphi_{j} \otimes \rho$ and $\operatorname{Res}_{K \cap K_{s}}^{K_{s}}\left(\varphi_{\jmath} \otimes \rho\right)^{s}$ are disjoint. We will prove that it suffices to show the restriction of these two representations to the subgroup $G / \Gamma=G / \Gamma \times\left\{e_{\Theta}\right\}$ of $K \cap K_{s}$ are disjoint, using the contrapositive. Suppose 
that $\sigma$ is a common subrepresentation of $\operatorname{Res}_{K \cap K_{s}}^{K} \varphi_{\jmath} \otimes \rho$ and $\operatorname{Res}_{K \cap K_{s}}^{K_{s}}\left(\varphi_{\jmath} \otimes \rho\right)^{s}$. We are restricting $\varphi_{\jmath} \otimes \rho$ and $\left(\varphi_{\jmath} \otimes \rho\right)^{s}$ to the subgroup $G / \Gamma \rtimes\left(\Theta_{\varphi_{\jmath}} \cap \tau\left(\Theta_{\varphi_{\jmath}}\right) \tau^{-1}\right)$, so we can consider the restriction $\operatorname{Res}_{G / \Gamma}^{K \cap K_{s}} \sigma$ of $\sigma$ to the subgroup $G / \Gamma$ of $K \cap K_{s}$. Obviously this is a common subrepresentation of both $\operatorname{Res}_{G / \Gamma}^{K \cap K_{s}} \operatorname{Res}_{K \cap K_{s}}^{K} \varphi_{j} \otimes \rho$ and $\operatorname{Res}_{G / \Gamma}^{K \cap K_{s}} \operatorname{Res}_{K \cap K_{s}}^{K_{s}}\left(\varphi_{\jmath} \otimes \rho\right)^{s}$.

Thus, we have shown if the restrictions of $\operatorname{Res}_{K \cap K_{s}}^{K}\left(\varphi_{\jmath} \otimes \rho\right)$ and $\operatorname{Res}_{K \cap K_{s}}^{K_{s}}\left(\varphi_{\jmath} \otimes \rho\right)^{s}$ to $G / \Gamma$ are disjoint, then $\operatorname{Res}_{K \cap K_{s}}^{K}\left(\varphi_{\jmath} \otimes \rho\right)$ and $\operatorname{Res}_{K_{n} K_{s}}^{K_{s}}\left(\varphi_{\jmath} \otimes \rho\right)^{s}$ are disjoint.

Consider $\left(x \Gamma, e_{\Theta}\right) \in G / \Gamma \times\left\{e_{\Theta}\right\}$ and $v \in V_{\jmath, \rho}$. Then

$$
\begin{aligned}
\left(\operatorname{Res}_{K_{\cap} K_{s}}^{K}\left(\varphi_{\jmath} \otimes \rho\right)\right)\left(x \Gamma, e_{\Theta}\right) v & =\left(\left(\varphi_{\jmath} \otimes \rho\right)\left(x \Gamma, e_{\Theta}\right)\right) v \\
& =\varphi_{\jmath}(x \Gamma) v
\end{aligned}
$$

Note that $s^{-1}\left(x \Gamma, e_{\Theta}\right) s=\left(\tau^{-1}(x) \Gamma, e_{\Theta}\right)$. Thus

$$
\begin{aligned}
\operatorname{Res}_{K \cap K_{s}}^{K_{s}}\left(\varphi_{\jmath} \otimes \rho\right)^{s}\left(x \Gamma, e_{\Theta}\right) v & =\left(\varphi_{\jmath} \otimes \rho\right)^{s}\left(x \Gamma, e_{\Theta}\right) v \\
& =\varphi_{\jmath} \otimes \rho\left(\tau^{-1}(x) \Gamma, e_{\Theta}\right) v \\
& =\tau \cdot \varphi_{\jmath}(x \Gamma) v
\end{aligned}
$$

As $s \notin G / \Gamma \rtimes \Theta_{\varphi_{\jmath}}, \tau \notin \Theta_{\varphi_{\jmath}}$, so $\tau \cdot \varphi_{\jmath} \neq \varphi_{\jmath}$. Therefore, $\operatorname{Res}_{K \cap K_{s}}^{K} \varphi_{\jmath} \otimes \rho\left(x \Gamma, e_{\Theta}\right) v \neq$ $\operatorname{Res}_{K \cap K_{s}}^{K_{s}}\left(\varphi_{\jmath} \otimes \rho\right)^{s}\left(x \Gamma, e_{\Theta}\right) v$ holds true for some $x \Gamma \in G / \Gamma$ and some non-zero $v \in V_{\jmath, \rho}$. This proves that the restrictions of $\operatorname{Res}_{K \cap K_{s}}^{K} \varphi_{J} \otimes \rho$ and $\operatorname{Res}_{K \cap K_{s}}^{K_{s}}\left(\varphi_{J} \otimes \rho\right)^{s}$ to $G / \Gamma$ are disjoint, which shows that $\operatorname{Res}_{K \cap K_{s}}^{K} \varphi_{\jmath} \otimes \rho$ and $\operatorname{Res}_{K \cap K_{s}}^{K_{s}}\left(\varphi_{\jmath} \otimes \rho\right)^{s}$ are disjoint. Since $\varphi_{\jmath} \otimes \rho$ is irreducible by Proposition 6.1.1, we conclude by Mackey's Criterion that $\psi_{3, \rho}$ is irreducible. 


\subsection{The Classification of all Irreducible Represen- tations of $G / \Gamma \rtimes \Theta$}

In $\S 6.2$ we showed that representations of the form $\psi_{3, \rho}$ of $G / \Gamma \rtimes \Theta$ are irreducible. We will prove that representations of this form are inequivalent and exhaust all of the possible irreducible representations of $G / \Gamma \rtimes \Theta$. We begin by discussing the structure of the induced space with respect to $\varphi_{\jmath} \otimes \rho, W_{\varphi_{j} \otimes \rho}^{G / \Gamma \rtimes \Theta}$, which was defined in $\S 6.2$.

Let $\left\{\theta_{1}, \ldots, \theta_{m_{\jmath}}\right\}$ be a complete set of coset representatives for $\Theta_{\varphi_{j}} \backslash \Theta$ with $\theta_{1}=e_{\Theta}$. Simplify the notation by setting $W_{\jmath, \rho}=W_{\varphi_{\jmath} \otimes \rho}^{G / \Gamma \times \Theta}$. Since $\psi_{\jmath, \rho}$ is an irreducible representation (Theorem 6.2.3), the vector space it acts on, $W_{\jmath, \rho}$, must be finite-dimensional $([7], \S 5.1)$. Let $d_{\jmath, \rho}=\operatorname{dim}\left(W_{\jmath, \rho}\right)$. For each $1 \leqslant l \leqslant m_{\jmath}$, define $W_{\jmath, \rho}^{l}$ to be the set of functions $f: G / \Gamma \rtimes \Theta \rightarrow V_{\jmath, \rho}$ such that

$$
f(y \Gamma, \theta)= \begin{cases}\varphi_{\jmath}(y \Gamma) \rho\left(\theta^{\prime}\right) f\left(\Gamma, \theta_{l}\right) & \text { if } \theta=\theta^{\prime} \theta_{l} \text { for some } \theta^{\prime} \in \Theta_{\varphi_{\jmath}} \\ 0 & \text { otherwise }\end{cases}
$$

\section{Lemma 6.3.1.}

1) $W_{\jmath, \rho}^{l}$ is a subspace of $W_{\jmath, \rho}$.

2) $W_{\jmath, \rho}^{l} \simeq V_{\jmath, \rho}$ as vector spaces.

3) $W_{\jmath, \rho}=\oplus_{l=1}^{m_{\jmath}} W_{\jmath, \rho}^{l}$.

4) $\operatorname{Res}_{G / \Gamma}^{G / \Gamma \times \Theta} \psi_{3, \rho}$ is equivalent to $\oplus_{l=1}^{m_{3}}\left(\oplus_{l=1}^{d \imath m V_{3, \rho}} \theta_{l}^{-1} \cdot \varphi_{\jmath}\right)$.

5) $\left.\operatorname{Res}_{\Theta_{\varphi_{j}}}^{G / \Gamma \times \Theta} \psi_{3, \rho}\right|_{W_{3, \rho}^{1}}$ is equivalent to $\rho$.

\section{Proof:}

1) That $W_{\jmath, \rho}^{l}$ is closed under addition, closed under scalar multiplication, and $0 \epsilon$ $W_{\jmath, \rho}^{l}$, are all clear. What is left to show is that $W_{\jmath, \rho}^{l} \subseteq W_{\jmath \rho}$ Suppose $f \in W_{\jmath, \rho}^{l}$, 
$(y \Gamma, \theta) \in G / \Gamma \rtimes \Theta$ and $(x \Gamma, \tau) \in G / \Gamma \rtimes \Theta_{\varphi_{\jmath}}$. First we consider the case where $\theta=\theta^{\prime} \theta_{l}$, where $\theta^{\prime} \in \Theta_{\varphi_{j}}$. Then $\tau \theta=\tau \theta^{\prime} \theta_{l}$, so that

$$
\begin{aligned}
f((x \Gamma, \tau)(y \Gamma, \theta)) & =f(x \tau(y) \Gamma, \tau \theta) \\
& =\varphi_{\jmath}(x \Gamma) \varphi_{\jmath}(\tau(y) \Gamma) \rho\left(\tau \theta^{\prime}\right) f\left(\Gamma, \theta_{l}\right) \\
& =\varphi_{\jmath}(x \Gamma) \tau^{-1} \cdot \varphi_{\jmath}(y \Gamma) \rho(\tau) \rho\left(\theta^{\prime}\right) f\left(\Gamma, \theta_{l}\right) \\
& =\varphi_{\jmath}(x \Gamma) \rho(\tau) \varphi_{\jmath}(y \Gamma) \rho\left(\theta^{\prime}\right) f\left(\Gamma, \theta_{l}\right) \quad \text { since } \tau^{-1} \in \Theta_{\varphi_{\jmath}} \\
& =\varphi_{\jmath} \otimes \rho(x \Gamma, \tau) f(y \Gamma, \theta) .
\end{aligned}
$$

If $\theta$ cannot be written as $\theta^{\prime} \theta_{l}$, then $\tau \theta$ cannot be either. Thus:

$$
f((x \Gamma, \tau)(y \Gamma, \theta))=f(x \tau(y) \Gamma, \tau \theta)=0=\left(\varphi_{\jmath} \otimes \rho\right)(x \Gamma, \tau) f(y \Gamma, \theta)
$$

Therefore we have $f \in W_{3, \rho}$.

2) Define $T_{l}: W_{\jmath, \rho}^{l} \rightarrow V_{\jmath, \rho}$ by $T_{l}(f)=f\left(\Gamma, \theta_{l}\right)$. It is clear that $T_{l}$ is a linear map. For $v \in V_{\jmath, \rho}$, define $f_{v} \in W_{\jmath, \rho}^{l}$ by

$$
f_{v}(y \Gamma, \theta)= \begin{cases}\varphi_{\jmath}(y \Gamma) \rho\left(\theta^{\prime}\right) v & \text { if } \theta=\theta^{\prime} \theta_{l} \text { for some } \theta^{\prime} \in \Theta_{\varphi_{\jmath}} \\ 0 & \text { otherwise }\end{cases}
$$

Since $f_{v}\left(\Gamma, \theta_{l}\right)=v$, by construction $f_{v} \in W_{\jmath, \rho}^{l}$. Let $S_{l}: V_{\jmath, \rho} \rightarrow W_{\jmath, \rho}^{l}$ be the map $S_{l}(v)=f_{v}$. It is easy to check that $S_{l}$ is also a linear map.

Let $f \in W_{\jmath, \rho}^{l}$. Then taking $v=f\left(\Gamma, \theta_{l}\right)$ above we see that

$$
f_{f\left(\Gamma, \theta_{l}\right)}(y \Gamma, \theta)=\left\{\begin{array}{ll}
\varphi_{\jmath}(y \Gamma) \rho\left(\theta^{\prime}\right) f\left(\Gamma, \theta_{l}\right) & \text { if } \theta=\theta^{\prime} \theta_{l} \text { for some } \theta^{\prime} \in \Theta_{\varphi_{3}} \\
0 & \text { otherwise }
\end{array} .\right.
$$

Thus $S_{l}\left(T_{l}(f)\right)=S_{l}\left(f\left(\Gamma, \theta_{l}\right)\right)=f_{f\left(\Gamma, \theta_{l}\right)}=f$. If $v \in V_{\jmath, \rho}$, then $f_{v}\left(\Gamma, \theta_{l}\right)=v$, so 
$T_{l}\left(S_{l}(v)\right)=T_{l}\left(f_{v}\right)=f_{v}\left(\Gamma, \theta_{l}\right)=v$. Therefore $S_{l}=T_{l}^{-1}$, making $T_{l}$ an invertible linear map from $W_{j, \rho}^{l}$ to $V_{\jmath, \rho}$.

3) Suppose $f \in W_{\jmath, \rho}$. Define $f_{l}: G / \Gamma \rtimes \Theta \rightarrow V_{j, \rho}$ by

$$
f_{l}(y \Gamma, \theta)=\left\{\begin{array}{ll}
f(y \Gamma, \theta) & \text { if } \theta=\theta^{\prime} \theta_{l} \text { for some } \theta^{\prime} \in \Theta_{\varphi_{j}} \\
0 & \text { otherwise }
\end{array} .\right.
$$

Note that $f_{l}\left(y \Gamma, \theta_{l}\right)=f\left(y \Gamma, \theta_{l}\right)$. Suppose that $\theta=\theta^{\prime} \theta_{l}$ for some $\theta^{\prime} \in \Theta_{\varphi_{j}}$. Let $y \Gamma \in G / \Gamma$. Then

$$
\begin{aligned}
f_{l}(y \Gamma, \theta) & =f(y \Gamma, \theta) \\
& =f\left(y \Gamma, \theta^{\prime} \theta_{l}\right) \\
& =f\left(\left(y \Gamma, \theta^{\prime}\right)\left(\Gamma, \theta_{l}\right)\right) \\
& =\varphi_{j}(y \Gamma) \rho\left(\theta^{\prime}\right) f\left(\Gamma, \theta_{l}\right) \\
& =\varphi_{j}(y \Gamma) \rho\left(\theta^{\prime}\right) f_{l}\left(\Gamma, \theta_{l}\right) .
\end{aligned}
$$

Thus $f_{l} \in W_{j, \rho}^{l}$. Suppose that $(y \Gamma, \theta) \in G / \Gamma \rtimes \Theta$. We can write $\theta=\theta^{\prime} \theta_{r}$, where $\theta^{\prime} \in \Theta_{\varphi_{j}}$ and $1 \leqslant r \leqslant m_{j}$ are unique. We have that

$$
\sum_{l=1}^{m_{3}} f_{l}(y \Gamma, \theta)=f_{r}(y \Gamma, \theta)=f(y \Gamma, \theta)
$$

Therefore $f=\sum_{l=1}^{m_{j}} f_{l}$, showing $W_{j}=\oplus_{l=1}^{m_{j}} W_{j, \rho}^{l}$.

4) Suppose $f \in W_{j, \rho}^{l}, x \Gamma \in G / \Gamma$, and $(y \Gamma, \theta) \in G / \Gamma \rtimes \Theta$. First suppose $\theta=\theta^{\prime} \theta_{l}$ 
for some $\theta^{\prime} \in \Theta_{\varphi_{3}}$. Then

$$
\begin{aligned}
\left(\psi_{\jmath, \rho}\left(x \Gamma, e_{\Theta}\right) f\right)(y \Gamma, \theta) & =f\left((y \Gamma, \theta)\left(x \Gamma, e_{\Theta}\right)\right) \\
& =f(y \theta(x) \Gamma, \theta) \\
& =\varphi_{\jmath}(y \theta(x) \Gamma) \rho\left(\theta^{\prime}\right) f\left(\Gamma, \theta_{l}\right) \\
& =\varphi_{\jmath}\left(\theta^{\prime}\left(\theta_{l}(x)\right) \Gamma\right) \varphi_{\jmath}(y \Gamma) \rho\left(\theta^{\prime}\right) f\left(\Gamma, \theta_{l}\right) \\
& =\theta^{\prime-1} \cdot \varphi_{\jmath}\left(\theta_{l}(x) \Gamma\right) \varphi_{\jmath}(y \Gamma) \rho\left(\theta^{\prime}\right) f\left(\Gamma, \theta_{l}\right) \\
& =\varphi_{\jmath}\left(\theta_{l}(x) \Gamma\right) f(y \Gamma, \theta) \\
& =\theta_{l}^{-1} \cdot \varphi_{\jmath}(x \Gamma) f(y \Gamma, \theta) .
\end{aligned}
$$

In the case where $\theta$ is not equal to $\theta^{\prime} \theta_{l}$ for some $\theta^{\prime} \in \Theta_{\varphi_{j}}$, we have

$$
\begin{aligned}
\left(\psi_{\jmath, \rho}\left(x \Gamma, e_{\Theta}\right) f\right)(y \Gamma, \theta) & =f\left((y \Gamma, \theta)\left(x \Gamma, e_{\Theta}\right)\right) \\
& =f(y \theta(x) \Gamma, \theta) \\
& =0 \\
& =\theta_{l}^{-1} \cdot \varphi_{\jmath}(x \Gamma) \varphi_{\jmath}(x \Gamma) f(y \Gamma, \theta) .
\end{aligned}
$$

Therefore, $\left.\operatorname{Res}_{G / \Gamma}^{G / \Gamma \times \Theta} \psi_{\jmath, \rho}\right|_{W_{j, \rho}^{l}}=\left(\theta_{l}^{-1} \cdot \varphi_{\jmath}\right) I_{W_{\jmath, \rho}^{l}}$. This means that the representation $\operatorname{Res}_{G / \Gamma}^{G / \Gamma \times \Theta} \psi_{\jmath, \rho}$ and $\oplus_{l=1}^{m_{\jmath}}\left(\oplus_{l=1}^{d 2 m V_{J, \rho}} \theta_{l}^{-1} \cdot \varphi_{\jmath}\right)$ are equivalent.

5) Suppose $f \in W_{3, \rho}^{1}$ and $\tau \in \Theta_{\varphi_{3}}$. Consider the map $T_{1}$, the special case that $l=1$ of the map $T_{l}$ defined in 2). Then

$$
\begin{aligned}
\left(\rho(\tau) T_{1}\right)(f) & =\rho(\tau) f\left(\Gamma, e_{\Theta}\right) \\
& =f(\Gamma, \tau) \\
& =f\left(\left(\Gamma, e_{\Theta}\right)(\Gamma, \tau)\right) \\
& =\left(\psi_{\jmath, \rho}(\Gamma, \tau) f\right)\left(\Gamma, e_{\Theta}\right) \\
& =T_{1}\left(\psi_{\jmath, \rho}(\Gamma, \tau) f\right)
\end{aligned}
$$


Therefore $T_{1}$ is an intertwining map of $\left.\operatorname{Res}_{\Theta_{\varphi_{j}}}^{G / \Gamma \times \Theta} \psi_{\jmath, \rho}\right|_{W_{1, \rho}^{1}}$ and $\rho$, proving that those two representations are equivalent.

Theorem 6.3.2. Let $\rho^{\prime}: \Theta_{\varphi_{k}} \rightarrow G L\left(V_{k, \rho^{\prime}}\right)$ be an irreducıble representation of $\Theta_{\varphi_{k}}$, where $V_{k, \rho^{\prime}}$ us a finıte-dimensional complex vector space. If $\psi_{\jmath, \rho}$ is equivalent to $\psi_{k, \rho^{\prime}}$, then $\jmath=k$ and $\rho$ is equivalent to $\rho^{\prime}$.

Proof: Suppose $\jmath \neq k$. Then $\varphi_{\jmath}$ and $\varphi_{k}$ are from different $\Theta$-orbits. Thus $\theta \cdot \varphi_{\jmath} \nsucc$ $\theta^{\prime} \cdot \varphi_{k}$ for all $\theta, \theta^{\prime} \in \Theta$.

If $\psi_{\jmath, \rho} \sim \psi_{k, \rho^{\prime}}$, then $\operatorname{Res}_{G / \Gamma}^{G / \Gamma \times \Theta} \psi_{\jmath, \rho} \sim \operatorname{Res}_{G / \Gamma}^{G / \Gamma \times \Theta} \psi_{k, \rho^{\prime}}$. By (4) of Lemma 6.3.1, $\operatorname{Res}_{G / \Gamma}^{G / \Gamma \rtimes \Theta} \psi_{\jmath, \rho} \sim$ $\oplus_{l=1}^{m_{\jmath}}\left(\oplus_{l=1}^{d m V_{J, \rho}} \theta_{l}^{-1} \cdot \varphi_{\jmath}\right)$ and $\operatorname{Res}_{G / \Gamma}^{G / \Gamma \times \Theta} \psi_{k, \rho^{\prime}} \sim \oplus_{l=1}^{t_{k}}\left(\oplus_{l=1}^{\operatorname{dim}_{k, \rho^{\prime}}} \tau_{l}^{-1} \cdot \varphi_{k}\right)$, where $\left\{\tau_{1}, \ldots, \tau_{t_{k}}\right\}$ is a complete set of coset representatives for $\Theta_{\varphi_{k}} \backslash \Theta$. The uniqueness of a decomposition into irreducible representations given by Maschke's Theorem ([19], §1.4) is up to isomorphısm and reordering, as proved in $([19], \S 2.3)$ for the case of a finite group. It is mentioned in $([19], \S 4.3)$ that this also is true for compact groups. Therefore, we can conclude that $\theta_{l}^{-1} \cdot \varphi_{\jmath} \sim \tau_{l}^{-1} \cdot \varphi_{k}$ for some appropriate $\imath, l$. This implies that $\jmath=k$, using the contrapositive of the first statement.

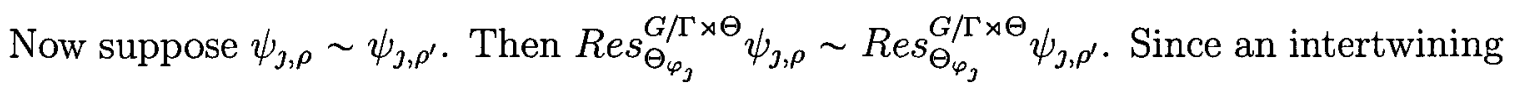
map between $\psi_{\jmath, \rho}$ and $\psi_{\jmath, \rho^{\prime}}$ will send $W_{\jmath, \rho}^{1}$ to $W_{\jmath, \rho^{\prime}}^{1}$, we have that $\left.\operatorname{Res}_{\Theta_{\varphi_{\jmath}}}^{G / \Gamma \rtimes \Theta_{\jmath, \rho}}\right|_{W_{\jmath, \rho}^{1}} \sim$ $\left.\operatorname{Res}_{\Theta_{\varphi_{j}}^{G / \Gamma \rtimes \Theta}}^{G /{ }^{\prime} \rho^{\prime}}\right|_{W_{\jmath \rho^{\prime}}^{1}}$. This implies, by (5) of Lemma 631 , that $\rho \sim \rho^{\prime}$.

For a finite-dimensional representation $\pi$ of a compact group $H$, let $\chi_{\pi}: H \rightarrow \mathbb{C}$ be the defined by $\chi_{\pi}(x)=\operatorname{tr}(\pi(x))$. The function $\chi_{\pi}$ is called the character of 
$\pi$. We can define an inner product on the space of characters of finite-dimensional representations of $H$ given by

$$
\left\langle\chi_{\pi_{1}}, \chi_{\pi_{2}}\right\rangle=\int_{H} \chi_{\pi_{1}}(x) \overline{\chi_{\pi_{2}}(x)} d x
$$

where integration is taken with respect to a Haar measure on $H$ that has been normalized to 1 . For two irreducible representations $\pi_{1}$ and $\pi_{2}$, we have that $\left\langle\chi_{\pi_{1}}, \chi_{\pi_{2}}\right\rangle=1$ if and only if $\pi_{1}$ and $\pi_{2}$ are equivalent. Also, we have $\left\langle\chi_{\pi_{1}}, \chi_{\pi_{2}}\right\rangle=0$ whenever $\pi_{1}$ and $\pi_{2}$ are inequivalent. These are proven in $([19], \S 2.3)$ in the case of a finite group, and mentioned in $([19], \S 4.3)$ that this proof carries over to the case of a compact group.

Theorem 6.3.3. Every irreducible representation of $G / \Gamma \rtimes \Theta$ is isomorphic to $\psi_{j, \rho}$ for some $j \in J$ and some irreducible representation $\rho$ of $\Theta_{\varphi_{j}}$.

Proof: Let $\sigma: G / \Gamma \rtimes \Theta \rightarrow G L(U)$ be an irreducible representation of $G / \Gamma \rtimes \Theta$. According to $([7], \S 5.1), \sigma$ is a finite-dimensional representation. Without loss of generality, $\operatorname{Res}_{G / \Gamma}^{G / \Gamma \times \Theta} \sigma$ has $\varphi_{j}$ as a subrepresentation for some $1 \leqslant j \leqslant m$. This means that the subspace $U_{\varphi_{\jmath}}=\left\{u \in U: \sigma\left(y, e_{\Theta}\right) u=\varphi_{\jmath}(y) u \forall y \in G / \Gamma\right\}$ is non-zero.

We show that $U_{\varphi^{\prime}}$ is invariant under $\sigma(e \Gamma, \theta)$ when $\theta \in \Theta_{\varphi_{j}}$. Let $\theta \in \Theta_{\varphi_{3}}, u \in U_{\varphi_{3}}$ and $y \Gamma \in G / \Gamma$. Then

$$
\begin{aligned}
\sigma\left(y \Gamma, e_{\Theta}\right) \sigma(e \Gamma, \theta) u & =\sigma(y \Gamma, \theta) u \\
& =\sigma(e \Gamma, \theta) \sigma\left(\theta^{-1}(y) \Gamma, e_{\Theta}\right) u \\
& =\sigma(e \Gamma, \theta) \varphi_{\jmath}\left(\theta^{-1}(y) \Gamma\right) u \\
& =\sigma(e \Gamma, \theta) \theta \cdot \varphi_{\jmath}(y \Gamma) u \\
& =\varphi_{\jmath}(y \Gamma) \sigma(e \Gamma, \theta) u
\end{aligned}
$$

Thus $\sigma(e \Gamma, \theta) u \in U_{\varphi_{\jmath}}$. Therefore $\sigma(e \Gamma, \theta) U_{\varphi_{\jmath}} \subseteq U_{\varphi_{\jmath}}$ for all $\theta \in \Theta_{\varphi_{\jmath}}$. 
Let $U_{\varphi_{3}}^{\prime}$ be a non-zero subspace of $U_{\varphi_{3}}$ of minimal dimension such that $\sigma(e \Gamma, \theta) U_{\varphi_{3}}^{\prime} \subseteq$ $U_{\varphi_{j}}^{\prime}$ for all $\theta \in \Theta_{\varphi_{j}}$. Define $\rho: \Theta_{\varphi_{\jmath}} \rightarrow G L\left(U_{\varphi_{j}}^{\prime}\right)$ by $\rho(\theta) u=\sigma(e \Gamma, \theta) u$ for all $\theta \in \Theta_{\varphi_{3}}$ and all $u \in U_{\varphi_{j}}^{\prime}$. If $\rho$ was not irreducible, then there would exist a proper non-zero subspace of $W$ of $U_{\varphi}^{\prime}$, such that $\rho(\theta) W=\sigma(e \Gamma, \theta) W \subseteq W$, which contradicts the minimality of $U_{\varphi_{3}}^{\prime}$. Thus $\rho$ is irreducible. For $(y \Gamma, \theta) \in G / \Gamma \rtimes \Theta_{\varphi_{\jmath}}$ and $u \in U_{\varphi_{\jmath}}^{\prime}$, we have

$$
\begin{aligned}
\left(\varphi_{\jmath} \otimes \rho\right)(y \Gamma, \theta) u & =\varphi_{\jmath}(y \Gamma) \rho(\theta) u \\
& =\varphi_{\jmath}(y \Gamma) \sigma(e \Gamma, \theta) u \\
& =\sigma\left(y \Gamma, e_{\Theta}\right) \sigma(e \Gamma, \theta) u \\
& =\sigma(y \Gamma, \theta) u
\end{aligned}
$$

Therefore $\varphi_{\jmath} \otimes \rho=\left.\operatorname{Res}_{G / \Gamma \times \Theta_{\varphi_{j}}}^{G / \Gamma \times \Theta}\right|_{U_{\varphi_{j}}^{\prime}}$. Thus, $\varphi_{\jmath} \otimes \rho$ is a subrepresentation of $\operatorname{Res}_{G / \Gamma \times \Theta_{\varphi_{j}}^{G / \Gamma \times \Theta}}^{G} \sigma$. By the unique decomposition of $\operatorname{Res}_{G / \Gamma \times \Theta_{\varphi_{j}}^{G}}^{G / \Gamma \times \varphi}$ into a direct sum of irreducible representations, we must have

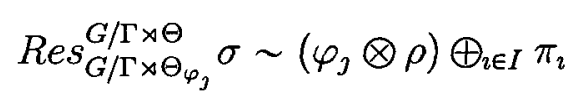

where $I$ is a finite indexing set and $\pi_{\imath}$ is irreducible for each $i \in I$. As both $\pi_{\imath}$ and $\varphi_{\jmath} \otimes \rho$ are irreducible representations of $G / \Gamma \rtimes \Theta_{\varphi_{\jmath}}$, we must have

$$
\left\langle\chi_{\varphi_{j} \otimes \rho}, \chi_{\pi_{2}}\right\rangle \in\{0,1\}
$$

for every $i \in I$. Therefore, we have that

$$
\begin{aligned}
\left\langle\chi_{\varphi_{j} \otimes \rho}, R e s_{G / \Gamma \rtimes \Theta_{\varphi_{j}}}^{G / \Gamma \rtimes \Theta} \chi_{\sigma}\right\rangle & =\left\langle\chi_{\varphi_{\jmath} \otimes \rho}, \chi_{\varphi_{j} \otimes \rho}\right\rangle+\sum_{\imath \in I}\left\langle\chi_{\varphi_{\jmath} \otimes \rho}, \chi_{\pi_{\imath}}\right\rangle \\
& \geqslant\left\langle\chi_{\varphi_{\jmath} \otimes \rho}, \chi_{\varphi_{j} \otimes \rho}\right\rangle \\
& =1 .
\end{aligned}
$$

Since both $\sigma$ and $\psi_{\jmath, \rho}$ are irreducible representations of $G / \Gamma \rtimes \Theta$, we must have 
$\left\langle\chi_{\psi_{3, \rho}}, \chi_{\sigma}\right\rangle \in\{0,1\}$. By Frobenius Reciprocity $([19], \S 7.2)$, we have

$$
\left\langle\chi_{\varphi_{j} \otimes \rho}, \operatorname{Res}_{G / \Gamma \times \Theta_{\varphi_{j}}}^{G / \Gamma \times \Theta} \chi_{\sigma}\right\rangle=\left\langle\chi_{\psi_{3, \rho}}, \chi_{\sigma}\right\rangle
$$

The left hand side is $\geqslant 1$ and the right hand side is $\leqslant 1$, meaning that $\left\langle\chi_{\psi_{3, \rho}}, \chi_{\sigma}\right\rangle=1$. Therefore $\psi_{3, \rho}$ and $\sigma$ are equivalent.

Recall that $J$ is the index set for our complete set of orbit representatives $\left\{\varphi_{J}: j \in J\right\}$.

Corollary 6.3.4. The complete set of all irreducible representations of $G / \Gamma \rtimes \Theta$ is

$$
\left\{\psi_{j, \rho}: j \in J, \rho \text { is an irreducible representation of } \Theta_{\varphi_{\jmath}}\right\} \text {. }
$$

Proof: This follows from Theorem 6.2.3, Thm 6.3.2 and Theorem 6.3.3.

\subsection{Computing the Traces of the Irreducible Rep- resentations}

We now will compute the trace values for each irreducible representation of $G / \Gamma \rtimes \Theta$. We do this so that we may later compute the trace of the regular representation in Chapter 7. Recall from $\S 6.3$ that $\left\{\theta_{1}, \ldots, \theta_{m_{3}}\right\}$ is a complete set of coset representatives for $\Theta_{\varphi_{j}} \backslash \Theta$ with $\theta_{1}=e_{\Theta}$. For every $\theta \in \Theta$, there exists a permutation $\sigma_{\theta} \in S_{m}$ such that $\Theta_{\varphi_{j}} \theta_{l} \theta=\Theta_{\varphi_{j}} \theta_{\sigma_{\theta}(l)}$ for all $1 \leqslant l \leqslant m_{\jmath}$. Thus, there exists $\tau_{l} \in \Theta_{\varphi_{j}}$ such that $\theta_{l} \theta=\tau_{l} \theta_{\sigma_{\theta}(l)}$. Note that $\tau_{l}$ depends on $\theta$. 
Lemma 6.4.1. Suppose $\theta \in \Theta, 1 \leqslant k \leqslant m_{\jmath}$ and $f \in W_{\jmath, \rho}^{k}$ (§6.3). Then $\psi_{\jmath, \rho}(\Gamma, \theta) f \in$ $W_{\jmath, \rho}^{\sigma_{\theta}^{-1}(k)}$.

Proof: Taking the value of $\psi_{\jmath, \rho}(\Gamma, \theta) f$ at $\left(\Gamma, \theta_{l}\right)$, we get

$$
\begin{aligned}
\left(\psi_{\jmath, \rho}(\Gamma, \theta) f\right)\left(\Gamma, \theta_{l}\right) & =f\left(\Gamma, \theta_{l} \theta\right) \\
& =f\left(\Gamma, \tau_{l} \theta_{\sigma_{\theta}(l)}\right)
\end{aligned}
$$

Therefore we get that

$$
\left(\psi_{\jmath, \rho}(\Gamma, \theta) f\right)\left(\Gamma, \theta_{l}\right)= \begin{cases}\rho\left(\tau_{l}^{\prime}\right) f\left(\Gamma, \theta_{k}\right) & \text { if } \tau_{l} \theta_{\sigma_{\theta}(l)}=\tau_{l}^{\prime} \theta_{k} \text { for some } \tau_{l}^{\prime} \in \Theta_{\varphi_{\jmath}} \\ 0 & \text { otherwise }\end{cases}
$$

But $\tau_{l} \theta_{\sigma_{\theta}(l)}=\tau_{l}^{\prime} \theta_{k}$ for some $\tau_{l}^{\prime} \in \Theta_{\varphi_{\jmath}}$ if and only if $\Theta_{\varphi_{\jmath}} \theta_{\sigma_{\theta}(l)}=\Theta_{\varphi_{j}} \theta_{k}$ which means that $\sigma_{\theta}(l)=k$.

If $\sigma_{\theta}(l)=k$, then $\tau_{l} \theta_{\sigma_{\theta}(l)}=\tau_{l}^{\prime} \theta_{k}$ implies that $\tau_{l}=\tau_{l}^{\prime}$. Thus

$$
\left(\psi_{\jmath, \rho}(\Gamma, \theta) f\right)\left(\Gamma, \theta_{l}\right)= \begin{cases}\rho\left(\tau_{l}\right) f\left(\Gamma, \theta_{k}\right) & \text { if } \sigma_{\theta}(l)=k \\ 0 & \text { otherwise }\end{cases}
$$

Suppose $\alpha \in \Theta$. Then $\alpha=\theta^{\prime} \theta_{l}$ for some $l$ and $\theta^{\prime} \in \Theta_{\varphi_{j}}$. Suppose that $\sigma_{\theta}(l)=k$. Then

$$
\begin{aligned}
\left(\psi_{\jmath, \rho}(\Gamma, \theta) f\right)(y \Gamma, \alpha) & =f\left(y \Gamma, \theta^{\prime} \theta_{l} \theta\right) \\
& =f\left(y \Gamma, \theta^{\prime} \tau_{l} \theta_{k}\right) \\
& =\varphi_{\jmath}(y \Gamma) \rho\left(\theta^{\prime} \tau_{l}\right) f\left(\Gamma, \theta_{k}\right) \\
& =\varphi_{\jmath}(y \Gamma) \rho\left(\theta^{\prime}\right)\left(\psi_{3, \rho}(\Gamma, \theta) f\right)\left(\Gamma, \theta_{l}\right)
\end{aligned}
$$

Therefore we have

$\left(\psi_{\jmath, \rho}(\Gamma, \theta) f\right)(y \Gamma, \alpha)= \begin{cases}\varphi_{\jmath}(y \Gamma) \rho\left(\theta^{\prime}\right)\left(\psi_{\jmath, \rho}(\Gamma, \theta) f\right)\left(\Gamma, \theta_{l}\right) & \text { if } \alpha=\theta^{\prime} \theta_{\sigma_{\theta}^{-1}(k)} \text { for some } \theta^{\prime} \in \Theta_{\varphi_{3}} \\ 0 & \text { otherwise }\end{cases}$ 
This proves that $\psi_{3, \rho}(\Gamma, \theta) f \in W_{\jmath, \rho}^{\sigma_{\theta}^{-1}(k)}$.

Define $\rho^{\theta_{k}}: \theta_{k}^{-1} \Theta_{\varphi_{\jmath}} \theta_{k} \rightarrow G L\left(V_{\jmath, \rho}\right)$ by $\rho^{\theta_{k}}(\theta)=\rho\left(\theta_{k} \theta \theta_{k}^{-1}\right)$. We now compute the trace of $\psi_{3, \rho}(\Gamma, \theta)$.

Theorem 6.4.2. For $(y \Gamma, \theta) \in G / \Gamma \rtimes \Theta$, the trace of $\psi_{3, \rho}(y \Gamma, \theta)$ is given by

$$
\operatorname{tr}\left(\psi_{\jmath, \rho}(y \Gamma, \theta)\right)=\sum_{k \in \in \theta_{k}^{-1} \Theta_{\varphi_{\jmath}} \theta_{k}}\left(\theta_{k}^{-1} \cdot \varphi_{\jmath}\right)(y \Gamma) \operatorname{tr}\left(\rho^{\theta_{k}}(\theta)\right)
$$

Proof: Recall Lemma 6.3.1 (3), which stated that $W_{3, \rho}=\oplus_{l=1}^{m_{\jmath}} W_{\jmath, \rho}^{l}$. For $1 \leqslant k \leqslant m$, let $\left\{f_{1}^{k}, \ldots, f_{d_{k}}^{k}\right\}$ be a basis for $W_{\jmath, \rho}^{k}$, where $d_{k}=\operatorname{dim}\left(W_{\jmath, \rho}^{k}\right)$. Consider the matrix of $\psi_{\jmath, \rho}(\Gamma, \theta)$ in terms of the basis $\left\{f_{1}^{1}, \ldots, f_{d_{1}}^{1}, f_{1}^{2}, \ldots, f_{d_{m}}^{m}\right\}$. The $d_{k} \times d_{k}$ block corresponding to $W_{\jmath, \rho}^{k}$ in this matrix can have non-zero entries if and only if there exists $f \in W_{\jmath, \rho}^{k}$ such that $\psi_{\jmath, \rho}(\Gamma, \theta) f \in W_{\jmath, \rho}^{k}$, which implies that $\sigma_{\theta}(k)=k$ by Lemma 6.4.1. Note that $\sigma_{\theta}(k)=k$ if and only if $\Theta_{\varphi_{j}} \theta_{k} \theta=\Theta_{\varphi_{3}} \theta_{k}$ if and only if $\theta_{k} \theta \theta_{k}^{-1} \in \Theta_{\varphi_{j}}$. Therefore we have that

$$
\operatorname{tr} \psi_{\jmath, \rho}(\Gamma, \theta)=\left.\sum_{\left\{k \theta_{k} \theta \theta_{k}^{-1} \in \Theta_{\varphi_{j}}\right\}} \operatorname{tr} \psi_{\jmath, \rho}(\Gamma, \theta)\right|_{W_{\jmath, \rho}^{k}}
$$

Suppose $\theta_{k} \theta \theta_{k}^{-1} \in \Theta_{\varphi_{\jmath}}, y \Gamma, x \Gamma \in G / \Gamma$ and $f \in W_{\jmath, \rho}^{k}$. Then

$$
\begin{aligned}
\left(\psi_{3, \rho}(y \Gamma, \theta) f\right)\left(x \Gamma, \theta_{k}\right) & =f\left(x \theta_{k}(y) \Gamma, \theta_{k} \theta \theta_{k}^{-1} \theta_{k}\right) \\
& =\varphi_{\jmath}(x \Gamma) \theta_{k}^{-1} \cdot \varphi_{\jmath}(y \Gamma) \rho^{\theta_{k}}(\theta) f\left(\Gamma, \theta_{k}\right) \\
& =\theta_{k}^{-1} \cdot \varphi_{\jmath}(y \Gamma) \rho^{\theta_{k}}(\theta) f\left(x \Gamma, \theta_{k}\right)
\end{aligned}
$$

Therefore $\psi_{\jmath, \rho}(y \Gamma, \theta) f=\theta_{k}^{-1} \cdot \varphi_{\jmath}(y \Gamma) \rho^{\theta_{k}}(\theta) f$, which shows that

$$
\left.\operatorname{tr} \psi_{\jmath, \rho}(y \Gamma, \theta)\right|_{W_{\jmath, \rho}^{k}}=\theta_{k}^{-1} \cdot \varphi_{\jmath}(y \Gamma) \operatorname{tr}\left(\rho^{\theta_{k}}(\theta)\right)
$$


This implies that we have the equation

$$
\operatorname{tr} \psi_{j, \rho}(y \Gamma, \theta)=\sum_{k: \theta \in \theta_{k}^{-1} \Theta_{\varphi_{j}} \theta_{k}} \theta_{k}^{-1} \cdot \varphi_{j}(y \Gamma) \operatorname{tr}\left(\rho^{\theta_{k}}(\theta)\right)
$$

for the trace of an irreducible representation of $G / \Gamma \rtimes \Theta$ at $(y \Gamma, \theta)$.

\subsection{Examples}

We look at two examples to help demonstrate the main ideas from this chapter.

\section{Example:}

Consider $G=\mathbb{Z}$ as a group under addition with the discrete topology. Let $\Gamma=n \mathbb{Z}$, so that $G / \Gamma=\mathbb{Z} / n \mathbb{Z} \cong \mathbb{Z}_{n}$. Let $\mu_{\mathbb{Z}}$ be the counting measure. Then the quotient measure $\mu_{\mathbb{Z} / n \mathbb{Z}}$ (See $\S 3.1$ ) on $\mathbb{Z} / n \mathbb{Z}$ will be the counting measure as well.

In $\S 2.2$ we saw that the group of irreducible characters on $\mathbb{Z}_{n}$ is the set $\widehat{\mathbb{Z}_{n}}=$ $\left\{\varphi_{0}, \varphi_{1}, \ldots, \varphi_{n-1}\right\}$, where $\varphi_{k}(\bar{m})=e^{\frac{2 k \pi r m}{n}}$.

Let $\Theta=A u t(\mathbb{Z})=\{i d, \theta\}$, where $i d$ is the identity automorphism and $\theta$ is the automorphism $\theta(m)=-m$. Note that $n \mathbb{Z}$ is stable under $\Theta$.

We now take the specific case where $n=3$. Since the only non-abelian group of order six is $S_{3}$ up to isomorphism ([1], §7.7), we have $\mathbb{Z}_{3} \rtimes \Theta \cong S_{3}$.

$\left\{\Theta \cdot \varphi_{0}, \Theta \cdot \varphi_{1}\right\}$ is the set of distinct character orbits, since $\Theta \cdot \varphi_{0}=\left\{\varphi_{0}\right\}$ and $\Theta \cdot \varphi_{1}=\left\{\varphi_{1}, \varphi_{2}\right\}$. The stabilizer subgroups are $\Theta_{\varphi_{0}}=\Theta$ and $\Theta_{\varphi_{1}}=\{i d\}$. There are two inequivalent irreducible representations for $\Theta, \rho_{0}$ and $\rho_{1}$, where $\rho_{0}$ is the triv- 
ial representation and $\rho_{1}$ is the sign character given by $\rho_{1}(\theta)=-1$. The subgroup $\{i d\}$ of $\Theta$ has only the trivial representation, which we denote by 1 .

By Corollary 6.3.4, the complete set inequivalent irreducible representations for $\mathbb{Z}_{3} \rtimes \Theta$ is $\psi_{0, \rho_{0}}, \psi_{0, \rho_{1}}$ and $\psi_{1,1}$. The first two are extensions of the trivial character on $\mathbb{Z}_{3}$ to $\mathbb{Z}_{3} \rtimes \Theta$, where specifically $\psi_{0, \rho_{0}}$ is the trivial character, while $\psi_{0, \rho_{1}}(\bar{m}, \tau)=\rho_{1}(\tau)$ for all $\bar{m} \in \mathbb{Z}_{3}$.

$\psi_{1,1}=\operatorname{In} d_{\mathbb{Z}_{3}}^{\mathbb{Z}_{3} \times \Theta} \varphi_{1} \otimes 1$ is a two-dimensional representation, since

$$
\operatorname{deg}\left(\psi_{1,1}\right)=\left[\mathbb{Z}_{3} \rtimes \Theta: \mathbb{Z}_{3}\right]=2 .
$$

The complete set of coset representatives for $\{i d\} \backslash \Theta$ is $\left\{\theta_{1}=i d, \theta_{2}=\theta\right\}$. By Theorem 6.4 .2

$$
\operatorname{tr}\left(\psi_{1, \mathbf{1}}(\bar{m}, \tau)\right)=\sum_{k: \tau \in \theta_{k}^{-1} \Theta_{\varphi_{1}} \theta_{k}} \theta_{k}^{-1} \cdot \varphi_{1}(\bar{m}) \operatorname{tr}\left(\mathbf{1}^{\theta_{k}}(\tau)\right)
$$

for $(\bar{m}, \tau) \in \mathbb{Z}_{3} \rtimes \Theta$. Since $\Theta_{\varphi_{1}}=\{i d\}$, the group $\theta_{k}^{-1} \Theta_{\varphi_{1}} \theta_{k}$ is trivial. Therefore the sum on the right is over both $k=1$ and $k=2$ when $\tau=i d$, and there is no sum when $\tau=\theta$. In other words, $\operatorname{tr}\left(\psi_{1,1}(\bar{m}, \theta)\right)=0$. Since 1 is trivial and one-dimensional, $\operatorname{tr}\left(\mathbf{1}^{\theta_{k}}(\tau)\right)=1$. Thus, for $\bar{m} \in \mathbb{Z}_{3}$

$$
\operatorname{tr}\left(\psi_{1,1}(\bar{m}, i d)\right)=\varphi_{1}(\bar{m})+\varphi_{1}(-\bar{m})=e^{\frac{2 \pi \imath m}{3}}+e^{\frac{4 \pi i m}{3}}
$$

\section{Example:}

Let $G=\mathbb{R}$ under the usual topology, and $\Gamma=\mathbb{Z}$. We saw in $\S 2.1$ that $\mathbb{R} / \mathbb{Z}$ is a compact group isomorphic to the circle group $\mathbb{T}$. Let $\mu_{\mathbb{R}}$ be the usual Borel measure on $\mathbb{R}$.

The group of irreducible characters is the set $\widehat{\mathbb{T}}=\left\{\varphi_{k}: k \in \mathbb{Z}\right\}$, where $\varphi_{k}(x)=e^{2 \pi i k x}$ (§2.2). 
Let $\Theta=\{i d, \theta\}$, where $i d$ is the identity automorphism and $\theta$ is the automorphism $\theta(x)=-x$. Note that $\mathbb{Z}$ is stable under $\Theta$.

We need to find a complete set of distinct orbits. For $x+\mathbb{Z} \in \mathbb{R} / \mathbb{Z}$, we have that $\theta \cdot \varphi_{k}(x+\mathbb{Z})=\varphi_{k}(-x+\mathbb{Z})=e^{-2 \pi \imath k x}$. Therefore $\theta \cdot \varphi_{k}=\varphi_{-k}$. Thus a complete set of orbits is $\left\{\Theta \cdot \varphi_{k}: k \geqslant 0\right\}$.

The stabilizer subgroups are $\Theta_{\varphi_{0}}=\Theta$ and $\Theta_{\varphi_{k}}=\{i d\}$ when $k>0$. There are two inequivalent irreducible representations of $\Theta, \rho_{0}$ and $\rho_{1}$, where $\rho_{0}$ is the trivial representation and $\rho_{1}$ is the sign character. The trivial group $\{i d\}$ has only the trivial representation 1 .

Therefore the complete set inequivalent irreducible representations for $\mathbb{R} / \mathbb{Z} \rtimes \Theta$ is

$$
\left\{\psi_{0, \rho_{0}}, \psi_{0, \rho_{1}}\right\} \cup\left\{\psi_{k, \mathbf{1}}: k>0\right\}
$$

where the first two are extensions of the trivial character on $\mathbb{R} / \mathbb{Z}$ to $\mathbb{R} / \mathbb{Z} \rtimes \Theta$. More specifically $\psi_{0, \rho_{0}}$ is the trivial character, while $\psi_{0, \rho_{1}}(x, \tau)=\rho_{1}(\tau)$ for all $x \in \mathbb{T}$.

For $k>0, \psi_{k, \mathbf{1}}=\operatorname{Ind} d_{\mathbb{T}}^{\mathbb{T} \times \Theta}\left(\varphi_{k} \otimes \mathbf{1}\right)$ is a two-dimensional representation, since $\operatorname{deg}\left(\psi_{k, \mathbf{1}}\right)=$ $[\mathbb{R} / \mathbb{Z} \rtimes \Theta: \mathbb{R} / \mathbb{Z}]=2$. The complete set of coset representatives for $\{i d\} \backslash \Theta$ is $\left\{\theta_{1}=\right.$ $\left.i d, \theta_{2}=\theta\right\}$. By Theorem 6.4.2, for $(x+\mathbb{Z}, \tau) \in \mathbb{R} / \mathbb{Z} \rtimes \Theta$

$$
\operatorname{tr}\left(\psi_{k, \mathbf{1}}(x+\mathbb{Z}, \tau)\right)=\sum_{m} \theta_{\tau \in \theta_{m}^{-1} \Theta_{\varphi_{k}} \theta_{m}} \theta_{m}^{-1} \cdot \varphi_{k}(x+\mathbb{Z}) \operatorname{tr}\left(\mathbf{1}^{\theta_{m}}(\tau)\right)
$$

Since $\Theta_{\varphi_{k}}=\{i d\}$, the group $\theta_{m}^{-1} \Theta_{\varphi_{k}} \theta_{m}$ is trivial. Therefore the sum on the right is over both $m=1$ and $m=2$ when $\tau=i d$ and there is no sum when $\tau=\theta$. Therefore, $\operatorname{tr}\left(\psi_{k, 1}(x+\mathbb{Z}, \theta)=0\right.$ for all $x+\mathbb{Z} \in \mathbb{R} / \mathbb{Z}$. Since $\mathbf{1}$ is trivial and one-dimensional, 
$\operatorname{tr}\left(\mathbf{1}^{\theta_{m}}(i d)\right)=1$. Thus, for $x+\mathbb{Z} \in \mathbb{R} / \mathbb{Z}$

$$
\operatorname{tr}\left(\psi_{k, 1}(x+\mathbb{Z}, i d)\right)=\varphi_{k}(x+\mathbb{Z})+\varphi_{k}(-x+\mathbb{Z})=e^{2 \pi \imath k x}+e^{-2 \pi \imath k x}=2 \cos (2 \pi i k x)
$$




\section{Chapter 7}

\section{Extension of the Poisson}

\section{Summation Formula}

We continue to work under the assumption that $G$ is a second countable LCA group with a discrete subgroup $\Gamma$ such that $G / \Gamma$ is compact, and that $\Theta$ is a finite subgroup of $A u t(G)$. We carry over the definition of the representations $\psi_{j, \rho}$ of $G / \Gamma \rtimes \Theta$, defined in Chapter 6 , where we proved that representations of this form exhaust all the irreducible representations of $G / \Gamma \rtimes \Theta$. We also determined a formula for the traces of these representations, given in Theorem 6.4.2. The right regular representation $\bar{R}$ of $G / \Gamma \rtimes \Theta$ was defined in Chapter 5. In this chapter, we prove a version of the PeterWeyl Theorem, which states that $\bar{R}$ is unitarily equivalent to $\bigoplus_{j, \rho} d_{j, \rho} \psi_{3, \rho}$. We will first define the representation $d_{j, \rho} \psi_{j, \rho}$ of $G / \Gamma \rtimes \Theta$, where $d_{j, \rho}$ is the dimension of $W_{j, \rho}$, a vector space defined in Chapter 6 . As its name suggests, it acts as $d_{j, \rho}$ copies of $\psi_{j, \rho}$, and the vector space it acts on is $\operatorname{End}\left(W_{j, \rho}\right)$, which isomorphic to $d_{j, \rho}$ copies of $W_{j, \rho}$ as a vector space. For $f \in C(G / \Gamma \rtimes \Theta)$, we define bounded linear operators $\psi_{j, \rho}(f)$ and $d_{j, \rho} \psi_{j, \rho}(f)$ on $W_{j, \rho}$ in a similar way to how we defined $\bar{R}_{f}$ in Chapter 5 . We will determine the traces of both of these linear operators, and discover that they relate to one another in a way the notation would suggest - that $\operatorname{tr}\left(d_{j, \rho} \psi_{j, \rho}(f)\right)=d_{j, \rho} \operatorname{tr}\left(\psi_{j, \rho}(f)\right)$. This will all work towards proving that, for $f \in C(G / \Gamma \rtimes \Theta) * C(G / \Gamma \rtimes \Theta)$, the trace 
of $\bar{R}_{f}$ is equal to the sum of $d_{\jmath, \rho} \operatorname{tr}\left(\psi_{\jmath, \rho}(f)\right)$ over all $j \in J$ and all irreducible representations $\rho$ of $\Theta_{\varphi_{j}}$. This, combined with the formula for $\bar{R}_{f}$ given in Corollary 5.5.2, give us an extension of the Poisson Summation Formula to $G \rtimes \Theta$. It is an extension in the sense that, when $\Theta$ is trivial, we recover the Poisson Summation Formula for $G / \Gamma$ given in Chapter 3.

\subsection{Computing the Trace of a Linear Operator on $W_{j, \rho}$}

Recall the irreducible representation $\psi_{\jmath, \rho}$ of $G / \Gamma \rtimes \Theta$ and the induced space $W_{\jmath, \rho}$, which were defined in $\S 6.2$ and $\S 6.3$ respectively. We will define a linear operator $\psi_{\jmath, \rho}(f)$ on $W_{\jmath, \rho}$ and compute its trace.

Recall that $W_{\jmath, \rho}$ is a finite-dimensional vector space $(\S 6.3)$, and that $d_{\jmath, \rho}=\operatorname{dim}\left(W_{\jmath, \rho}\right)$. Fix a basis $\beta$ of $W_{\jmath, \rho}$. For a function of the form $g: G / \Gamma \rtimes \Theta \rightarrow \operatorname{Aut}\left(W_{\jmath, \rho}\right)$, we can consider $g(x \Gamma, \tau)$ as a matrix in terms of the basis $\beta$. That is, $g(x \Gamma, \tau)=\left[g_{s, t}(x \Gamma, \tau)\right]$, where $g_{s, t}(x \Gamma, \tau) \in \mathbb{C}$ are the matrix entries. We can define the integral of $g$ with respect to $\beta$ by

$$
\int_{G / \Gamma \rtimes \Theta} g(x \Gamma, \tau) d(x \Gamma, \tau)=\left[\int_{G / \Gamma \rtimes \Theta} g_{s, t}(x \Gamma, \tau) d(x \Gamma, \tau)\right] .
$$

For $f \in C(G / \Gamma \rtimes \Theta)$, let $\psi_{\jmath, \rho}(f)$ be defined by

$$
\psi_{\jmath, \rho}(f)=\int_{G / \Gamma \rtimes \Theta} f(x \Gamma, \theta) \psi_{\jmath, \rho}(x \Gamma, \theta) d(x \Gamma, \theta) .
$$

where we are integrating over the matrix $f(x \Gamma, \theta) \psi_{\jmath, \rho}(x \Gamma, \theta)$ with respect to $\beta$. The function $\psi_{\jmath, \rho}$ is a $d_{\jmath, \rho} \times d_{\jmath, \rho}$ matrix, so we can view $\psi_{\jmath, \rho}(f)$ is a linear operator on $W_{\jmath, \rho}$, 
that is $\psi_{\jmath, \rho}(f): W_{\jmath, \rho} \rightarrow W_{\jmath, \rho}$.

Proposition 7.1.1. For $f \in C(G / \Gamma \rtimes \Theta)$, we have

$$
\operatorname{tr}\left(\psi_{\jmath, \rho}(f)\right)=\int_{G / \Gamma \rtimes \Theta} f(x \Gamma, \theta) \operatorname{tr}\left(\psi_{\jmath, \rho}(x \Gamma, \theta)\right) d(x \Gamma, \theta) .
$$

Proof: Since $W_{\jmath, \rho}$ is finite-dimensional, the trace of $\psi_{\jmath, \rho}(f)$ is given by

$$
\begin{aligned}
\operatorname{tr}\left(\psi_{\jmath, \rho}(f)\right) & =\operatorname{tr}\left(\int_{G / \Gamma \rtimes \Theta} f(x \Gamma, \theta) \psi_{\jmath, \rho}(x \Gamma, \theta) d(x \Gamma, \theta)\right) \\
& =\operatorname{tr}\left(\left[\int_{G / \Gamma \rtimes \Theta} f(x \Gamma, \theta)\left(\psi_{\jmath, \rho}\right)_{s, t}(x \Gamma, \theta) d(x \Gamma, \theta)\right]\right) \\
& =\sum_{s=1}^{d_{3, \rho}} \int_{G / \Gamma \rtimes \Theta} f(x \Gamma, \theta)\left(\psi_{\jmath, \rho}\right)_{s, s}(x \Gamma, \theta) d(x \Gamma, \theta) \\
& =\int_{G / \Gamma \rtimes \Theta} f(x \Gamma, \theta) \sum_{s=1}^{d_{3, \rho}}\left(\left(\psi_{\jmath, \rho}\right)_{s, s}(x \Gamma, \theta)\right) d(x \Gamma, \theta) \\
& =\int_{G / \Gamma \rtimes \Theta} f(x \Gamma, \theta) \operatorname{tr}\left(\psi_{\jmath, \rho}(x \Gamma, \theta)\right) d(x \Gamma, \theta) .
\end{aligned}
$$

Note that this value of the trace does not depend on our choice of basis $\beta$ for $W_{\jmath, \rho}$, since $\operatorname{tr}\left(\psi_{3, \rho}(x \Gamma, \tau)\right)$ is invariant under a change of basis. In Theorem 6.4.2, it was determined that

$$
\operatorname{tr}\left(\psi_{\jmath, \rho}(x \Gamma, \theta)\right)=\sum_{\{k} \sum_{\left.\theta \in \theta_{k}^{-1} \Theta_{\varphi_{\jmath}} \theta_{k}\right\}} \theta_{k}^{-1} \cdot \varphi_{\jmath}(x \Gamma) \operatorname{tr}\left(\rho^{\theta_{k}}(\theta)\right) .
$$

Therefore the trace of $\psi_{3, \rho}(f)$ is given by

$$
\operatorname{tr}\left(\psi_{\jmath, \rho}(f)\right)=\int_{G / \Gamma \rtimes \Theta} f(x \Gamma, \theta) \sum_{\left\{k \in \in \theta_{k}^{-1} \Theta_{\varphi_{\jmath}} \theta_{k}\right\}} \theta_{k}^{-1} \cdot \varphi_{\jmath}(x \Gamma) \operatorname{tr}\left(\rho^{\theta_{k}}(\theta)\right) d(x \Gamma, \theta) .
$$




\subsection{The Peter-Weyl Theorem}

We will define a representation $d_{3, \rho} \psi_{3, \rho}$ of $G / \Gamma \rtimes \Theta$, as well as an associated linear operator $d_{\jmath, \rho} \psi_{\jmath, \rho}(f)$ on $\operatorname{End}\left(W_{\jmath, \rho}\right)$. We also define the direct sum representation $\oplus_{\jmath, \rho} d_{\jmath, \rho} \psi_{\jmath, \rho}$ of $G / \Gamma \rtimes \Theta$. We will prove a particular version of the Peter-Weyl Theorem, showing that the representations $\bar{R}$ and $\oplus_{\jmath, \rho} d_{\jmath, \rho} \psi_{\jmath, \rho}$ are unitarily equivalent. In order to prove it, we will construct a unitary isomorphism and intertwining map $\alpha$ between $W=\bigoplus_{\jmath, \rho} \operatorname{End}\left(W_{\jmath, \rho}\right)$ and $L^{2}(G / \Gamma \rtimes \Theta)$.

Let $\left\{\mathcal{H}_{\imath}: i \in I\right\}$ be a family of Hilbert spaces. The Hulbert space direct sum, denoted by $\bigoplus_{\imath \in I} \mathcal{H}_{\imath}$, is the set of elements $h=\left(h_{\imath}\right)_{\imath \in I}$ of the vector space direct product such that $\sum_{\imath \in I}\left|h_{\imath}\right|$ is finite. The Hilbert space direct sum is also a Hilbert space ([17], $\S 12.9)$ with inner product

$$
\langle h, v\rangle=\sum_{\imath \in I}\left\langle h_{\imath}, v_{\imath}\right\rangle
$$

Recall that $d_{\jmath, \rho}=\operatorname{dim}\left(W_{3, \rho}\right)$. Fix an element $j \in J$, an irreducible representation $\rho$ of $\Theta_{\varphi_{\jmath}}$ and a basis $\left\{f_{1}, \ldots, f_{d_{\jmath, \rho}}\right\}$ of $W_{\jmath, \rho}$. For $1 \leqslant k, l \leqslant d_{\jmath, \rho}$, let $A_{k, l} \in \operatorname{End}\left(W_{\jmath, \rho}\right)$ be defined by

$$
A_{k, l}\left(\sum_{r=1}^{d_{\jmath, \rho}} c_{r} f_{r}\right)=c_{l} f_{k}
$$

where $\sum_{r=1}^{d_{j, \rho}} c_{r} f_{r} \in W_{\jmath, \rho}$. The matrix of $A_{k, l}$ with respect to the basis $\left\{f_{1}, \ldots, f_{d_{3, \rho}}\right\}$ is the matrix with a value of 1 in row $k$ and column $l$, and a value of 0 everywhere else. This matrix, a member of the vector space $M_{d_{j, \rho} \times d_{j, \rho}}(\mathbb{C})$, is denoted by $E_{k, l}$. The space $\operatorname{End}\left(W_{\jmath, \rho}\right)$ is a Hilbert space with Hilbert-Schmidt inner product $\langle A, B\rangle=\operatorname{tr}\left(A B^{*}\right)$ ([21], §6.6). It can be shown that $\left\{A_{k, l}: 1 \leqslant k, l \leqslant d\right\}$ is an orthonormal basis for $\operatorname{End}\left(W_{\jmath, \rho}\right)$, and thus $\operatorname{drm}\left(\operatorname{End}\left(W_{\jmath, \rho}\right)\right)=d_{\jmath, \rho}^{2}$. It also can be shown that $\operatorname{End}\left(W_{\jmath, \rho}\right)$ and the Hilbert space direct sum $\bigoplus_{\imath=1}^{d_{\jmath, \rho}} W_{\jmath, \rho}$ are isomorphic as inner product spaces. 
We can view elements of $\operatorname{End}\left(W_{\jmath, \rho}\right)$ as matrices with respect to our basis $\left\{A_{k, l}: 1 \leqslant k, l \leqslant d\right\}$ of $W_{\jmath, \rho}$. In this sense, define $d_{\jmath, \rho} \psi_{\jmath, \rho}: G / \Gamma \rtimes \Theta \rightarrow G L\left(\operatorname{End}\left(W_{\jmath, \rho}\right)\right)$ by

$$
\left(d_{\jmath, \rho} \psi_{\jmath, \rho}\right)(y \Gamma, \theta) A=A\left(\psi_{\jmath, \rho}(y \Gamma, \theta)\right)^{T}
$$

where $A \in \operatorname{End}\left(W_{\jmath, \rho}\right),(y \Gamma, \theta) \in G / \Gamma \rtimes \Theta$ and $T$ denotes the transpose. The space $G L\left(\operatorname{End}\left(W_{\jmath, \rho}\right)\right)$ is a topological space under the strong-operator topology ( $\left.\S 4.1\right)$. We check that $d_{\jmath, \rho} \psi_{\jmath, \rho}$ is a representation of $G / \Gamma \rtimes \Theta$. The continuity of $d_{\jmath, \rho} \psi_{\jmath, \rho}$ follows from the fact that matrix multiplication and transposing a matrix are both continuous functions on $\operatorname{End}\left(W_{3, \rho}\right)$. All that remains to be shown is that $d_{\jmath, \rho} \psi_{\jmath, \rho}$ preserves the group law. Suppose that $(y \Gamma, \theta)$ and $(x \Gamma, \tau)$ are elements of $G / \Gamma \rtimes \Theta$, and $A \in \operatorname{End}\left(W_{\jmath, \rho}\right)$. Then

$$
\begin{aligned}
\left(d_{\jmath, \rho} \psi_{\jmath, \rho}\right)(y \Gamma, \theta)\left(\left(d_{\jmath, \rho} \psi_{\jmath, \rho}\right)(x \Gamma, \tau) A\right) & =\left(d_{\jmath, \rho} \psi_{\jmath, \rho}\right)(y \Gamma, \theta)\left(A\left(\psi_{\jmath, \rho}(x \Gamma, \tau)\right)^{T}\right) \\
& =A\left(\psi_{\jmath, \rho}(x \Gamma, \tau)^{T}\right)\left(\psi_{\jmath, \rho}(y \Gamma, \theta)\right)^{T} \\
& =A\left(\psi_{\jmath, \rho}(y \Gamma, \theta) \psi_{\jmath, \rho}(x \Gamma, \tau)\right)^{T} \\
& =A\left(\psi_{\jmath, \rho}((y \Gamma, \theta)(x \Gamma, \tau))\right)^{T} \\
& =\left(d_{\jmath, \rho} \psi_{\jmath, \rho}\right)((y \Gamma, \theta),(x \Gamma, \tau)) .
\end{aligned}
$$

For $f \in C(G / \Gamma \rtimes \Theta)$, define $d_{\jmath, \rho} \psi_{\jmath, \rho}(f): \operatorname{End}\left(W_{\jmath, \rho}\right) \rightarrow \operatorname{End}\left(W_{\jmath, \rho}\right)$ by

$$
d_{\jmath, \rho} \psi_{\jmath, \rho}(f)=\int_{G / \Gamma \rtimes \Theta} f(x \Gamma, \tau)\left(d_{\jmath, \rho} \psi_{\jmath, \rho}\right)(x \Gamma, \tau) d(x \Gamma, \tau)
$$

where we are integrating over the matrix $f(x \Gamma, \tau) d_{\jmath, \rho} \psi_{\jmath, \rho}(x \Gamma, \tau)$ with respect to the fixed basis $\left\{A_{k, l}: 1 \leqslant k, l \leqslant d\right\}$ of $\operatorname{End}\left(W_{\jmath, \rho}\right)$. Clearly $d_{\jmath, \rho} \psi_{\jmath, \rho}(f)$ is a linear operator.

Let $W=\oplus_{\jmath, \rho} \operatorname{End}\left(W_{\jmath, \rho}\right)$, where the Hilbert space direct sum is taken over all $\jmath \in J$ and all equivalence classes of irreducible representations $\rho$ of $\Theta_{\varphi_{3}}$. Define the direct 
sum representation $\bigoplus_{\jmath, \rho} d_{\jmath, \rho} \psi_{\jmath, \rho}: G / \Gamma \rtimes \Theta \rightarrow A u t(W)$ by

$$
\left(\bigoplus_{\jmath, \rho} d_{\jmath, \rho} \psi_{\jmath, \rho}(y \Gamma, \theta)\right)\left(\bigoplus_{\jmath, \rho} A_{\jmath, \rho}\right)=\bigoplus_{\jmath, \rho} A_{\jmath, \rho} \psi_{\jmath, \rho}(y \Gamma, \theta)^{T}
$$

where $\bigoplus_{\jmath, \rho} A_{\jmath, \rho} \in W$ and $(y \Gamma, \theta) \in G / \Gamma \rtimes \Theta$.

Recall the representation $\bar{R}$ of $G / \Gamma \rtimes \Theta$ defined in $\S 6.3$. Our goal is to prove a particular version of the Peter-Weyl Theorem, which states that $\bar{R}$ and $\bigoplus_{\jmath, \rho} d_{\jmath, \rho} \psi_{\jmath, \rho}$ are unitarily equivalent. Before we can do that, we need to state another version of the Peter-Weyl Theorem which is proved in $([20], \S 7.10)$. Define the matrix coeffi$\operatorname{cient}\left[\psi_{3, \rho}\right]_{k, l} \in L^{2}(G / \Gamma \rtimes \Theta)$ by $\left[\psi_{\jmath, \rho}\right]_{k, l}(y \Gamma, \theta)=\left[\psi_{\jmath, \rho}(y \Gamma, \theta)\right]_{k, l}=\left\langle\psi_{\jmath, \rho}(y \Gamma, \theta) f_{k}, f_{l}\right\rangle$.

Theorem 7.2.1 (Peter-Weyl Theorem (Part 1)). The set $\left\{\sqrt{d_{\jmath, \rho}}\left[\psi_{\jmath, \rho}\right]_{k, l}: 1 \leqslant k, l \leqslant\right.$ $d_{\jmath, \rho}, j \in J, \rho$ is irreducible $\}$ is an orthonormal basis for $L^{2}(G / \Gamma \rtimes \Theta)$.

The proof of part one is omitted, as it involves high-level functional analysis. The second part of the theorem, which will be stated shortly, is easier to prove. Its proof mostly follows from part one, and is mainly an exercise in defining a unitary intertwining map between $W$ and $L^{2}(G / \Gamma \rtimes \Theta)$.

Theorem 7.2.2 (Peter-Weyl Theorem (Part 2)). The representations $\bigoplus_{\jmath, \rho} d_{\jmath, \rho} \psi_{\jmath, \rho}$ and $\bar{R}$ are unitarlly equivalent.

Proof: Consider the space $M_{d \times d}(\mathbb{C})$, which is a Hilbert space with inner product defined by $\langle A, B\rangle=\operatorname{tr}\left(A B^{*}\right)$, where $A, B \in M_{d \times d}(\mathbb{C})$. The map $\beta_{\jmath, \rho}: \operatorname{End}\left(W_{\jmath, \rho}\right) \rightarrow$ $M_{d \times d}(\mathbb{C})$ given by $\beta_{\jmath, \rho}\left(\sum_{k, l=1}^{d} c_{k, l} A_{k, l}\right)=\sum_{k, l=1}^{d} c_{k, l} E_{k, l}$ is a unitary isomorphism. This follows from the fact that $\operatorname{End}\left(W_{3, \rho}\right)$ has Hilbert-Schmidt inner product given by 
$\langle A, B\rangle=\operatorname{tr}\left(A B^{*}\right)$ for $A, B \in \operatorname{End}\left(W_{\jmath, \rho}\right)$, which gives the same value as the HilbertSchmidt inner product on $M_{d \times d}(\mathbb{C})$. Let $\epsilon_{\jmath, \rho}: M_{d \times d}(\mathbb{C}) \rightarrow L^{2}(G / \Gamma \rtimes \Theta)$ be given by $\epsilon_{\jmath, \rho}\left(\sum_{k, l=1}^{d} c_{k, l} E_{k, l}\right)=\sum_{k, l=1}^{d} \sqrt{d_{\jmath, \rho}} c_{k, l}\left[\psi_{\jmath, \rho}\right]_{k, l}$, and $\alpha_{\jmath, \rho}: \operatorname{End}\left(W_{\jmath, \rho}\right) \rightarrow L^{2}(G / \Gamma \rtimes \Theta)$ be given by $\alpha_{\jmath, \rho}=\epsilon_{\jmath, \rho} \circ \beta_{\jmath, \rho}$. One may combine the $\alpha_{\jmath, \rho}$ to form a map $\alpha: W \rightarrow$ $L^{2}(G / \Gamma \rtimes \Theta)$ by setting $\alpha\left(\oplus_{\jmath, \rho} A_{\jmath, \rho}\right)=\sum_{\jmath, \rho} \alpha_{\jmath, \rho}\left(A_{\jmath, \rho}\right)$. We will prove the theorem by showing the map $\alpha$ is an intertwining map and unitary isomorphism.

We first show that $\epsilon_{\jmath, \rho}: M_{d \times d}(\mathbb{C}) \rightarrow L^{2}(G / \Gamma \rtimes \Theta)$ is unitary. It suffices to show that it preserves the inner product on the basis $\left\{E_{k, l}\right\}$ of $M_{d \times d}(\mathbb{C})$.

$$
\begin{aligned}
\left\langle E_{k, l}, E_{k^{\prime}, l^{\prime}}\right\rangle & =\operatorname{tr}\left(E_{k, l} E_{k^{\prime}, l^{\prime}}^{*}\right) \\
& =\operatorname{tr}\left(E_{k, l} E_{l^{\prime}, k^{\prime}}\right) \\
& =\delta_{l, l^{\prime}} \delta_{k, k^{\prime}} \\
& =\left\langle\sqrt{d_{\jmath, \rho}}\left[\psi_{\jmath, \rho}\right]_{k, l}, \sqrt{d_{\jmath, \rho}}\left[\psi_{\jmath, \rho}\right]_{k^{\prime}, l^{\prime}}\right\rangle \\
& =\left\langle\epsilon_{\jmath, \rho}\left(E_{k, l}\right), \epsilon_{\jmath, \rho}\left(E_{k^{\prime}, l^{\prime}}\right)\right\rangle .
\end{aligned}
$$

Since $\beta_{\jmath, \rho}$ is unitary, the map $\alpha_{\jmath, \rho}$ is unitary. This shows that the map $\alpha$ on the Hilbert space direct sum $W$ is unitary.

The Peter-Weyl Theorem (Part 1) shows that $\alpha$ sends an orthonormal basis of $W$ to an orthonormal basis of $L^{2}(G / \Gamma \rtimes \Theta)$, verifying that $\alpha$ is a unitary isomorphism of vector spaces. All that remains to be shown is that $\alpha$ is an intertwining map. Since $\left\{A_{k, l}\right\}$ is a basis for $\operatorname{End}\left(W_{\jmath, \rho}\right)$, it is enough to show that, for a fixed $j \in J$ and irreducible representation $\rho$,

$$
\bar{R}(y \Gamma, \theta) \alpha_{\jmath, \rho}\left(A_{k, l}\right)=\alpha_{\jmath, \rho}\left(\bigoplus_{\jmath, \rho} d_{\jmath, \rho} \psi_{\jmath, \rho}(y \Gamma, \theta)\left(A_{k, l}\right)\right)
$$


We examine the left and right hand sides of this equation separately. The LHS equals

$$
\bar{R}(y \Gamma, \theta) \alpha_{\jmath, \rho}\left(A_{k, l}\right)=\sqrt{d_{\jmath, \rho}} \bar{R}(y \Gamma, \theta)\left[\psi_{\jmath, \rho}\right]_{k, l} .
$$

Consider $A_{k, l} \psi_{\jmath, \rho}(y \Gamma, \theta)^{T}$ as a matrix in terms of the basis $\left\{f_{1}, \ldots, f_{d}\right\}$ of $W_{\jmath, \rho}$. The matrix coefficients are given by the matrix multiplication formula

$$
\begin{aligned}
{\left[A_{k, l} \psi_{\jmath, \rho}(y \Gamma, \theta)^{T}\right]_{s, t} } & =\sum_{r=1}^{d}\left[A_{k, l}\right]_{s, r}\left[\psi_{\jmath, \rho}(y \Gamma, \theta)^{T}\right]_{r, t} \\
& =\sum_{r=1}^{d}\left[A_{k, l}\right]_{s, r}\left[\psi_{\jmath, \rho}(y \Gamma, \theta)\right]_{t, r} \\
& =\left\{\begin{array}{ll}
{\left[\psi_{\jmath, \rho}(y \Gamma, \theta)\right]_{t, l}} & \text { if } s=k \\
0 & \text { otherwise }
\end{array} .\right.
\end{aligned}
$$

Now, for the RHS,

$$
\begin{aligned}
\alpha_{\jmath, \rho}\left(\bigoplus_{\jmath, \rho} d_{\jmath, \rho} \psi_{\jmath, \rho}(y \Gamma, \theta)\left(A_{k, l}\right)\right) & =\epsilon_{\jmath, \rho} \beta_{\jmath, \rho}\left(A_{k, l} \psi_{\jmath, \rho}(y \Gamma, \theta)^{T}\right) \\
& =\epsilon_{\jmath, \rho}\left(\sum_{t=1}^{d}\left[\psi_{\jmath, \rho}(y \Gamma, \theta)\right]_{t, l} E_{k, t}\right) \\
& =\sqrt{d_{\jmath, \rho}} \sum_{t=1}^{d}\left[\psi_{\jmath, \rho}(y \Gamma, \theta)\right]_{t, l}\left[\psi_{\jmath, \rho}\right]_{k, t} .
\end{aligned}
$$

To show equality of the left and right hand sides, we apply both to an element $(x \Gamma, \tau)$ of $G / \Gamma \rtimes \Theta$. In the former case

$$
\sqrt{d_{\jmath, \rho}} \bar{R}(y \Gamma, \theta)\left[\psi_{\jmath, \rho}\right]_{k, l}(x \Gamma, \tau)=\sqrt{d_{\jmath, \rho}}\left[\psi_{\jmath, \rho}(x \Gamma, \tau)(y \Gamma, \theta)\right]_{k, l} .
$$

In the latter case

$$
\begin{aligned}
\sqrt{d_{\jmath, \rho}} \sum_{t=1}^{d}\left[\psi_{\jmath, \rho}(y \Gamma, \theta)\right]_{t, l}\left[\psi_{\jmath, \rho}\right]_{k, t}(x \Gamma, \tau) & =\sqrt{d_{\jmath, \rho}} \sum_{t=1}^{d}\left[\psi_{\jmath, \rho}(x \Gamma, \tau)\right]_{k, t}\left[\psi_{\jmath, \rho}(y \Gamma, \theta)\right]_{t, l} \\
& =\sqrt{d_{\jmath, \rho}}\left[\psi_{\jmath, \rho}(x \Gamma, \tau)(y \Gamma, \theta)\right]_{k, l} .
\end{aligned}
$$


Therefore $\bar{R}(y \Gamma, \theta) \alpha_{\jmath, \rho}\left(A_{k, l}\right)=\alpha_{\jmath, \rho}\left(\oplus_{\jmath, \rho} d_{\jmath, \rho} \psi_{\jmath, \rho}(y \Gamma, \theta)\left(A_{k, l}\right)\right)$, proving that $\alpha$ is an intertwining map.

\subsection{Extending the Poisson Summation Formula to $G \rtimes \Theta$}

Applying the version of the Peter-Weyl theorem proved in the previous section, for $f \in C_{c}(G \rtimes \Theta) * C_{c}(G \rtimes \Theta)$ we will determine a new formula for $\operatorname{tr}\left(R_{f}\right)=\operatorname{tr}\left(\bar{R}_{f_{\Gamma}}\right)$ as the sum of $d_{\jmath, \rho} \operatorname{tr}\left(\psi_{\jmath, \rho}(f)\right)$ over all $j \in J$ and equivalence classes of irreducible representations $\rho$ of $\Theta_{\varphi_{\jmath}}$. First, we will need to show that $\operatorname{tr}\left(d_{\jmath, \rho} \psi_{\jmath, \rho}(f)\right)=d_{\jmath, \rho} \operatorname{tr}\left(\psi_{3, \rho}(f)\right)$. We will combine our new formula for $\operatorname{tr}\left(R_{f}\right)$ with the one in Theorem 5.5.2 to give an extension of the Poisson Summation formula to $G \rtimes \Theta$.

Recall the representation $d_{\jmath, \rho} \psi_{\jmath, \rho}$ of $G / \Gamma \rtimes \Theta$ defined in $\S 7.2$. The next propositions shows us that its trace at $(y \Gamma, \theta)$ relates to $\psi_{\jmath, \rho}$ exactly as its notation suggests.

Proposition 7.3.1. $\operatorname{tr}\left(d_{\jmath, \rho} \psi_{\jmath, \rho}(y \Gamma, \theta)\right)=d_{\jmath, \rho} \operatorname{tr}\left(\psi_{\jmath, \rho}(y \Gamma, \theta)\right)$.

Proof: Recall that

$$
\left[A_{k, l} \psi_{\jmath, \rho}(y \Gamma, \theta)^{T}\right]_{s, t}= \begin{cases}{\left[\psi_{\jmath, \rho}(y \Gamma, \theta)\right]_{t, l}} & \text { if } s=k \\ 0 & \text { otherwise }\end{cases}
$$


This gives us that

$$
\begin{aligned}
{\left[A_{k, l} \psi_{\jmath, \rho}(y \Gamma, \theta)^{T} A_{l, k}\right]_{s, r} } & =\sum_{t=1}^{d}\left[A_{k, l} \psi_{\jmath, \rho}(y \Gamma, \theta)^{T}\right]_{s, t}\left[A_{l, k}\right]_{t, r} \\
& = \begin{cases}{\left[\psi_{\jmath, \rho}(y \Gamma, \theta)\right]_{l, l}} & \text { if } s=r=k \\
0 & \text { otherwise }\end{cases}
\end{aligned}
$$

For $(y \Gamma, \theta) \in G / \Gamma \rtimes \Theta$, we have

$$
\begin{aligned}
\operatorname{tr}\left(d_{\jmath, \rho} \psi_{\jmath, \rho}(y \Gamma, \theta)\right) & =\sum_{k=1}^{d_{\jmath, \rho}} \sum_{l=1}^{d_{\jmath, \rho}}\left\langle d_{\jmath, \rho} \psi_{\jmath, \rho}(y \Gamma, \theta) A_{k, l}, A_{l, k}\right\rangle \\
& =\sum_{k=1}^{d_{\jmath, \rho}} \sum_{l=1}^{d_{\jmath, \rho}} \operatorname{tr}\left(A_{k, l} \psi_{\jmath, \rho}(y \Gamma, \theta)^{T} A_{k, l}^{*}\right) \\
& =\sum_{k=1}^{d_{\jmath, \rho}} \sum_{l=1}^{d_{\jmath, \rho}} \sum_{s=1}^{d_{\jmath, \rho}}\left[A_{k, l} \psi_{\jmath, \rho}(y \Gamma, \theta)^{T} A_{l, k}\right]_{s, s} \\
& =\sum_{k=1}^{d_{\jmath, \rho}} \sum_{l=1}^{d_{\jmath, \rho}}\left[\psi_{\jmath, \rho}(y \Gamma, \theta)\right]_{l, l} \\
& =\sum_{k=1}^{d_{\jmath, \rho}} \operatorname{tr}\left(\psi_{\jmath, \rho}(y \Gamma, \theta)\right) \\
& =d_{\jmath, \rho} \operatorname{tr}\left(\psi_{\jmath, \rho}(y \Gamma, \theta)\right) .
\end{aligned}
$$

Only non-zero when $s=k$

Recall the linear operator $d_{\jmath, \rho} \psi_{\jmath, \rho}(f)$ defined in $\$ 7.2$. We will determine its trace value.

Corollary 7.3.2. For $f \in C(G / \Gamma \rtimes \Theta)$, we have $\operatorname{tr}\left(d_{\jmath, \rho} \psi_{\jmath, \rho}(f)\right)=d_{\jmath, \rho} \operatorname{tr}\left(\psi_{\jmath, \rho}(f)\right)$. 
Proof: Since $\operatorname{End}\left(W_{\jmath, \rho}\right)$ is finite dimensional with $\operatorname{dim}\left(\operatorname{End}\left(W_{\jmath, \rho}\right)\right)=d_{\jmath, \rho}^{2}$, the trace is given by

$$
\begin{aligned}
\operatorname{tr}\left(d_{\jmath, \rho} \psi_{\jmath, \rho}(f)\right) & =\operatorname{tr}\left(\int_{G / \Gamma \rtimes \Theta} f(x \Gamma, \theta) d_{\jmath, \rho} \psi_{\jmath, \rho}(x \Gamma, \theta) d(x \Gamma, \theta)\right) \\
& =\operatorname{tr}\left(\left[\int_{G / \Gamma \rtimes \Theta} f(x \Gamma, \theta)\left(d_{\jmath, \rho} \psi_{\jmath, \rho}\right)_{s, t}(x \Gamma, \theta) d(x \Gamma, \theta)\right]\right) \\
& =\sum_{s=1}^{d_{\jmath, \rho}^{2}} \int_{G / \Gamma \rtimes \Theta} f(x \Gamma, \theta)\left(d_{\jmath, \rho} \psi_{\jmath, \rho}\right)_{s, s}(x \Gamma, \theta) d(x \Gamma, \theta) \\
& =\int_{G / \Gamma \rtimes \Theta} f(x \Gamma, \theta) \sum_{s=1}^{d_{\jmath, \rho}^{2}}\left(\left(d_{\jmath, \rho} \psi_{\jmath, \rho}\right)_{s, s}(x \Gamma, \theta)\right) d(x \Gamma, \theta) \\
& =\int_{G / \Gamma \rtimes \Theta} f(x \Gamma, \theta) \operatorname{tr}\left(d_{\jmath, \rho} \psi_{\jmath, \rho}(x \Gamma, \theta)\right) d(x \Gamma, \theta) .
\end{aligned}
$$

By Proposition 7.3.1, we have

$$
\begin{aligned}
\operatorname{tr}\left(d_{\jmath, \rho} \psi_{\jmath, \rho}(f)\right) & =\int_{G / \Gamma \rtimes \Theta} f(x \Gamma, \theta) \operatorname{tr}\left(d_{\jmath, \rho} \psi_{\jmath, \rho}(x \Gamma, \theta)\right) d(x \Gamma, \theta) \\
& =d_{\jmath, \rho} \int_{G / \Gamma \rtimes \Theta} f(x \Gamma, \theta) \operatorname{tr}\left(\psi_{\jmath, \rho}(x \Gamma, \theta)\right) d(x \Gamma, \theta) \\
& =d_{\jmath, \rho} \operatorname{tr}\left(\psi_{\jmath, \rho}(f)\right) .
\end{aligned}
$$

We now prove the main result of this section, which gives a formula for the trace of the bounded linear operator $\bar{R}_{f}$ that was defined in $\S 5.4$. This formula is in a different form than the one given by Corollary 5.5.2.

Theorem 7.3.3. For $f \in L^{2}(G / \Gamma \rtimes \Theta) * L^{2}(G / \Gamma \rtimes \Theta), \bar{R}_{f}$ us trace class and

$$
\operatorname{tr}\left(\bar{R}_{f}\right)=\sum_{\jmath, \rho} d_{\jmath, \rho} \operatorname{tr}\left(\psi_{\jmath, \rho}(f)\right)
$$

where the sum on the right is taken over all $\jmath \in J$ and all equivalence classes of irreducible representations $\rho$ of $\Theta_{\varphi_{j}}$, or equivalently over all equivalence classes of 
ırreducıble representatıons of $G / \Gamma \rtimes \Theta$ (by Corollary 6.3.4). Since $\bar{R}_{f}$ is trace class, it follows that the sum on the right hand side is absolutely convergent.

Proof: The Peter-Weyl Theorem (Part 1) states that $\left\{\sqrt{d_{\jmath, \rho}}\left[\psi_{\jmath, \rho}\right]_{k, l}: 1 \leqslant k, l \leqslant\right.$ $\left.d_{\jmath, \rho}\right\}$ forms an orthonormal basis for $L^{2}(G / \Gamma \rtimes \Theta)$. Thus, for $(y \Gamma, \theta) \in G / \Gamma \rtimes \Theta$

$$
\begin{aligned}
\operatorname{tr}\left(\bar{R}_{f}\right) & =\sum_{\jmath, \rho} \sum_{k, l}\left\langle\sqrt{d_{\jmath, \rho}} \bar{R}_{f}\left[\psi_{\jmath, \rho}\right]_{k, l}, \sqrt{d_{\jmath, \rho}}\left[\psi_{\jmath, \rho}\right]_{k, l}\right\rangle \\
& =\sum_{\jmath, \rho} \sum_{k, l}\left\langle\int_{G / \Gamma \rtimes \Theta} f(x \Gamma, \tau) \sqrt{d_{\jmath, \rho}} \bar{R}(x \Gamma, \tau)\left[\psi_{\jmath, \rho}\right]_{k, l}, \sqrt{d_{\jmath, \rho}}\left[\psi_{\jmath, \rho}\right]_{k, l}\right\rangle
\end{aligned}
$$

The integral above may be pulled out of the inner product, as shown in greater generality in ([17], pg. 74). Therefore

$$
\operatorname{tr}\left(\bar{R}_{f}\right)=\sum_{\jmath, \rho} \sum_{k, l} \int_{G / \Gamma \times \Theta} f(x \Gamma, \tau)\left\langle\sqrt{d_{\jmath, \rho}} \bar{R}(x \Gamma, \tau)\left[\psi_{\jmath, \rho}\right]_{k, l}, \sqrt{d_{\jmath, \rho}}\left[\psi_{\jmath, \rho}\right]_{k, l}\right\rangle
$$

The Peter-Weyl Theorem (Part 2) showed that $\alpha$ is a unitary intertwining map. Therefore, we get

$$
\begin{aligned}
\operatorname{tr}\left(\bar{R}_{f}\right) & =\sum_{\jmath, \rho} \sum_{k, l} \int_{G / \Gamma \rtimes \Theta} f(x \Gamma, \tau)\left\langle d_{\jmath, \rho} \psi_{\jmath, \rho}(x \Gamma, \tau) A_{k, l}, A_{k, l}\right\rangle \\
& =\sum_{\jmath, \rho} \sum_{k, l}\left\langle\int_{G / \Gamma \rtimes \Theta} f(x \Gamma, \tau) d_{\jmath, \rho} \psi_{\jmath, \rho}(x \Gamma, \tau) A_{k, l}, A_{k, l}\right\rangle \\
& =\sum_{\jmath, \rho} \sum_{k, l}\left\langle d_{\jmath, \rho} \psi_{\jmath, \rho}(f) A_{k, l}, A_{k, l}\right\rangle \\
& =\sum_{\jmath, \rho} \operatorname{tr}\left(d_{\jmath, \rho} \psi_{\jmath, \rho}(f)\right) \\
& =\sum_{\jmath, \rho} d_{\jmath, \rho} \operatorname{tr}\left(\psi_{\jmath, \rho}(f)\right)
\end{aligned}
$$

by Proposition 7.3.2.

We have now extended the Poisson Summation formula to $G \rtimes \Theta$ 
Corollary 7.3.4. Suppose $f \in C_{c}(G \rtimes \Theta) * C_{c}(G \rtimes \Theta)$. Then we have

$$
|\Theta| \sum_{\gamma \in \Gamma} f\left(\gamma, e_{\Theta}\right)=\sum_{j, \rho} d_{j, \rho} \operatorname{tr}\left(\psi_{j, \rho}(f)\right)
$$

where the sum on the right is taken over all $j \in J$ and all equivalence classes of irreducible representations $\rho$ of $\Theta_{\varphi_{j}}$, and is absolutely convergent.

Proof: This follows immediately from Theorem 5.5.2 and Theorem 7.3.3.

\subsection{The Independence of the Traces from $\Theta$}

In $\S 7.3$, we combined Theorem 5.5.2 and Theorem 7.3.3 together to gain an extension of the Poisson Summation Formula to $G \rtimes \Theta$. In this section, we consider the special case of a function $f \in C_{c}(G \rtimes \Theta) * C_{c}(G \rtimes \Theta)$ which vanishes at any $(y \Gamma, \theta) \in G / \Gamma \rtimes \Theta$ with $\theta \neq e_{\Theta}$. For such a function, we confirm that the right hand side of the formula given by Theorem 7.3.3 can be simplified to give the right hand side of the formula given by Theorem 5.5.2. Using this, we simplify the formula given by Corollary 7.3.4 to have no dependence on the values $f \in C_{c}(G \rtimes \Theta) * C_{c}(G \rtimes \Theta)$ attains at non-identity elements of $\Theta$.

For $f \in C_{c}(G \rtimes \Theta)$, define $\tilde{f}: G \rtimes \Theta \rightarrow \mathbb{C}$ by

$$
\tilde{f}(x, \theta)=\left\{\begin{array}{ll}
f\left(x, e_{\Theta}\right) & \text { if } \theta=e_{\Theta} \\
0 & \text { otherwise }
\end{array} .\right.
$$

The function $\tilde{f}$ sends all values of $f$ with a non-trivial $\theta \in \Theta$ to 0 . It can be viewed as transforming a function in $C_{c}(G \rtimes \Theta)$ into a function in $C_{c}(G)$. 
Proposition 7.4.1. If $f=g * h$, where $g, h \in C_{c}(G \rtimes \Theta)$, then $\tilde{f} \in C_{c}(G \rtimes \Theta)$ * $C_{c}(G \rtimes \Theta)$.

Proof: For $\tau \in \Theta$, let $g_{\tau}, h_{\tau} \in C_{c}(G \rtimes \Theta)$ be defined by

$$
\begin{aligned}
& g_{\tau}(x, \theta)=\left\{\begin{array}{ll}
g\left(x, \tau^{-1}\right) & \text { if } \theta=e_{\Theta} \\
0 & \text { otherwise }
\end{array} .\right. \\
& h_{\tau}(x, \theta)=\left\{\begin{array}{ll}
h(\tau(x), \tau) & \text { if } \theta=e_{\Theta} \\
0 & \text { otherwise }
\end{array} .\right.
\end{aligned}
$$

A routine check shows these are continuous of compact support.

We claim that $\tilde{f}=\sum_{\tau \in \Theta} g_{\tau} * h_{\tau}$. Suppose $y \in G$. Then

$$
\begin{aligned}
\left(\sum_{\tau \in \Theta} g_{\tau} * h_{\tau}\right)\left(y, e_{\Theta}\right) & =\sum_{\tau \in \Theta} \int_{G \rtimes \Theta} g_{\tau}\left(\left(y, e_{\Theta}\right)\left(\theta^{-1}\left(x^{-1}\right), \theta^{-1}\right)\right) h_{\tau}(x, \theta) d(x, \theta) \\
& =\sum_{\tau \in \Theta} \int_{G} \sum_{\theta \in \Theta} g_{\tau}\left(y \theta^{-1}\left(x^{-1}\right), \theta^{-1}\right) h_{\tau}(x, \theta) d(x) \\
& =\sum_{\tau \in \Theta} \int_{G} g_{\tau}\left(y x^{-1}, e_{\Theta}\right) h_{\tau}\left(x, e_{\Theta}\right) d(x) \\
& \text { since } g_{\tau}, h_{\tau} \text { are only non-zero at } e_{\Theta} . \\
& =\sum_{\tau \in \Theta} \int_{G} g\left(y x^{-1}, \tau^{-1}\right) h(\tau(x), \tau) d(x) \\
& \text { Set } x_{1}=\tau(x) . \text { Note that } d\left(x_{1}\right)=d(x) . \\
& =\sum_{\tau \in \Theta} \int_{G} g\left(y \tau^{-1}\left(x_{1}^{-1}\right), \tau^{-1}\right) h\left(x_{1}, \tau\right) d\left(x_{1}\right) \\
& \text { Set } x=x_{1} \text { for convenience. } \\
& =\int_{G \rtimes \Theta} g\left(\left(y, e_{\Theta}\right)\left(\tau^{-1}\left(x^{-1}\right), \tau^{-1}\right)\right) h(x, \tau) d(x, \tau) \\
& =g * h\left(y, e_{\Theta}\right) \\
& =\tilde{f}\left(y, e_{\Theta}\right) .
\end{aligned}
$$


Let $\sigma \in \Theta$ be such that $\sigma \neq e_{\Theta}$. Then

$$
\begin{aligned}
\left(\sum_{\tau \in \Theta} g_{\tau} * h_{\tau}\right)(y, \sigma) & =\sum_{\tau \in \Theta} \int_{G \rtimes \Theta} g_{\tau}\left((y, \sigma)\left(\theta^{-1}\left(x^{-1}\right), \theta^{-1}\right)\right) h_{\tau}(x, \theta) d(x, \theta) \\
& =\sum_{\tau \in \Theta} \int_{G} \sum_{\theta \in \Theta} g_{\tau}\left(y \sigma\left(\theta^{-1}\left(x^{-1}\right)\right), \sigma \theta^{-1}\right) h_{\tau}(x, \theta) d(x) \\
& =\sum_{\tau \in \Theta} \int_{G} g_{\tau}\left(y x^{-1}, e_{\Theta}\right) h_{\tau}(x, \sigma) d(x)
\end{aligned}
$$

since $g_{\tau}$ is only non-zero at $e_{\Theta}$. As $\sigma \neq e_{\Theta}$, we must have $h_{\tau}(x, \sigma)=0$ for every $\tau \in \Theta$. This proves that

$$
\left(\sum_{\tau \in \Theta} g_{\tau} * h_{\tau}\right)(y, \sigma)=0=\tilde{f}(y, \sigma)
$$

Therefore $\tilde{f}=\sum_{\tau \in \Theta} g_{\tau} * h_{\tau} \in C_{c}(G \rtimes \Theta) * C_{c}(G \rtimes \Theta)$.

It should be noted that, in general we do not have $\tilde{f}=\widetilde{g} * \widetilde{h}$, which is why Proposition 7.4.1 had to be proved in such a complicated manner.

Corollary 7.4.2. If $f=g * h$, where $g, h \in C_{c}(G \rtimes \Theta)$, then $\tilde{f}_{\Gamma} \in L^{2}(G / \Gamma \rtimes \Theta)$ * $L^{2}(G / \Gamma \rtimes \Theta)$.

Proof: This follows from Proposition 7.4.1, which showed that $\tilde{f} \in C_{c}(G \rtimes \Theta)$ * $C_{c}(G \rtimes \Theta)$, and Proposition 5.4.5.

Corollary 7.4.3. If $f=g * h$, where $g, h \in C_{c}(G \rtimes \Theta)$, then $\bar{R}_{\tilde{f}_{\Gamma}}$ is trace class. 
Proof: This follows from the previous corollary and Theorem 5.4.2 which states that the space of functions such that $\bar{R}_{f}$ is trace class is $L^{2}(G / \Gamma \rtimes \Theta) * L^{2}(G / \Gamma \rtimes \Theta)$.

Proposition 7.4.4. If $f=g * h$, where $g, h \in C_{c}(G \rtimes \Theta)$, then $\operatorname{tr}\left(R_{f}\right)=\operatorname{tr}\left(\bar{R}_{f_{\mathrm{\Gamma}}}\right)=$ $\operatorname{tr}\left(\bar{R}_{\tilde{f}_{\Gamma}}\right)$.

Proof: By Theorem 5.5.2, we have

$$
\begin{aligned}
\operatorname{tr}\left(\overline{R_{f_{\Gamma}}}\right) & =f_{\Gamma}\left(\Gamma, e_{\Theta}\right) \\
& =\sum_{\gamma \in \Gamma} f\left(\gamma, e_{\Theta}\right) \\
& =\sum_{\gamma \in \Gamma} \tilde{f}\left(\gamma, e_{\Theta}\right) \\
& =\tilde{f}_{\Gamma}\left(\Gamma, e_{\Theta}\right) \\
& =\operatorname{tr}\left(\overline{R_{\tilde{f_{\Gamma}}}}\right) .
\end{aligned}
$$

The following theorem confirms that the formulas given by Theorem 5.5.2 and Theorem 7.3.3 for $\operatorname{tr}\left(R_{f}\right)=\operatorname{tr}\left(\bar{R}_{f_{\Gamma}}\right)$ coincide in the special case that $f \in C_{c}(G \rtimes \Theta)$ * $C_{c}(G \rtimes \Theta)$ vanishes away from the identity of $\Theta$.

Theorem 7.4.5. For $f \in C_{c}(G \rtimes \Theta) * C_{c}(G \rtimes \Theta)$, we have

$$
\sum_{\jmath, \rho} d_{\jmath, \rho} \operatorname{tr}\left(\psi_{\jmath, \rho}\left(\tilde{f}_{\Gamma}\right)\right)=|\Theta| \tilde{f}_{\Gamma}\left(e \Gamma, e_{\Theta}\right)
$$


Proof: First we compute $\operatorname{tr}\left(\psi_{\jmath, \rho}\left(\tilde{f}_{\Gamma}\right)\right)$ for $f \in C_{c}(G \rtimes \Theta) * C_{c}(G \rtimes \Theta)$.

$$
\begin{aligned}
\operatorname{tr}\left(\psi_{j, \rho}\left(\tilde{f}_{\Gamma}\right)\right) & =\int_{G / \Gamma \times \Theta} \tilde{f}_{\Gamma}(x \Gamma, \theta) \operatorname{tr}\left(\psi_{\jmath, \rho}(x \Gamma, \theta)\right) d(x \Gamma, \theta) \\
& =\sum_{\theta \in \Theta} \int_{G / \Gamma} \tilde{f}_{\Gamma}(x \Gamma, \theta) \operatorname{tr}\left(\psi_{j, \rho}(x \Gamma, \theta)\right) d(x \Gamma) \\
& =\int_{G / \Gamma} \tilde{f}_{\Gamma}\left(x \Gamma, e_{\Theta}\right) \operatorname{tr}\left(\psi_{j, \rho}\left(x \Gamma, e_{\Theta}\right)\right) d(x \Gamma) \\
& =\int_{G / \Gamma} \tilde{f}_{\Gamma}\left(x \Gamma, e_{\Theta}\right) \sum_{\left\{k: e_{\Theta} \theta_{k}^{-1} \Theta_{\varphi_{j}} \theta_{k}\right\}} \theta_{k}^{-1} \cdot \varphi_{\jmath}(x \Gamma) \operatorname{tr}\left(\rho^{\theta_{k}}\left(e_{\Theta}\right)\right) d(x \Gamma) \quad \text { by Theorem } 6.4 .2 \\
& =\int_{G / \Gamma} \tilde{f}_{\Gamma}\left(x \Gamma, e_{\Theta}\right) \sum_{k=1}^{m_{\jmath}} \varphi_{j}\left(\theta_{k}(x) \Gamma\right) \operatorname{tr}\left(\rho\left(e_{\Theta}\right)\right) d(x \Gamma) \\
& =\operatorname{deg}(\rho) \int_{G / \Gamma} \tilde{f}_{\Gamma}\left(x \Gamma, e_{\Theta}\right) \sum_{k=1}^{m_{\jmath}} \overline{\varphi_{j}\left(\theta_{k}\left(x^{-1}\right) \Gamma\right)} d(x \Gamma) \\
& =\operatorname{deg}(\rho) \sum_{k=1}^{m_{j}} \int_{G / \Gamma} \tilde{f}_{\Gamma}\left(x \Gamma, e_{\Theta}\right) \overline{\theta_{k}^{-1} \cdot \varphi_{\jmath}\left(x^{-1} \Gamma\right)} d(x \Gamma) \\
& =\operatorname{deg}(\rho) \sum_{k=1}^{m_{j}} \widehat{\tilde{f}_{\Gamma}\left(\theta_{k}^{-1} \cdot \varphi_{j}^{-1}\right) .}
\end{aligned}
$$

where we are considering $\tilde{f}_{\Gamma}$ as a function on $G / \Gamma$ defined by $\tilde{f}_{\Gamma}(x \Gamma)=\tilde{f}_{\Gamma}\left(x \Gamma, e_{\Theta}\right)$.

As vector spaces, we have $W_{j, \rho}=\oplus_{l=1}^{m_{j}} W_{j, \rho}^{l} \cong \oplus_{l=1}^{m_{j}} V_{j, \rho}$. Therefore $d_{j, \rho}=\operatorname{dim}\left(W_{j, \rho}\right)=$ $m_{j} \operatorname{dim}\left(V_{j, \rho}\right)=m_{j} \operatorname{deg}(\rho)$.

As mentioned in $\S 6.1,\left\{\Theta \cdot \varphi_{j}: j \in J\right\}$ is a complete set of distinct orbits for the action of $\Theta$ on $\widehat{G / \Gamma}$. It can easily be shown that $\left\{\Theta \cdot \varphi_{j}^{-1}: j \in J\right\}$ is also a complete set of distinct orbits. Also, $\Theta_{\varphi_{\jmath}}=\Theta_{\varphi_{j}^{-1}}$, as $\theta \in \Theta_{\varphi_{j}}$ if and only if $\varphi\left(\theta^{-1}(x) \Gamma\right)=\varphi(x \Gamma)$ for all $x \Gamma \in G / \Gamma$ if and only if $\overline{\varphi\left(\theta^{-1}(x) \Gamma\right)}=\overline{\varphi(x \Gamma)}$ for all $x \Gamma \in G / \Gamma$ if and only if $\varphi^{-1}\left(\theta^{-1}(x) \Gamma\right)=\varphi^{-1}(x \Gamma)$ for all $x \Gamma \in G / \Gamma$ if and only if $\theta \in \Theta_{\varphi_{3}^{-1}}$. Therefore, we have that

$$
\widehat{G / \Gamma}=\cup_{\jmath \in J} \Theta \cdot \varphi_{\jmath}^{-1}=\cup_{\jmath \in J} \Theta / \Theta_{\varphi_{\jmath}} \cdot \varphi_{\jmath}^{-1} .
$$


Recall from $\S 6.3$ that $\theta_{1}, \ldots, \theta_{m_{\jmath}}$ is a complete set of coset representatives for $\Theta_{\varphi_{\jmath}} \backslash \Theta$. It follows easily that $\theta_{1}^{-1}, \ldots, \theta_{m_{j}}^{-1}$ is a complete set of coset representatives for $\Theta / \Theta_{\varphi_{j}}$. This implies that

$$
\widehat{G / \Gamma}=\left\{\theta_{k}^{-1} \cdot \varphi_{j}^{-1}: j \in J, 1 \leqslant k \leqslant m_{j}\right\}
$$

Since $\Theta_{\varphi_{j}}$ is a finite group, we know from the representation theory of finite groups that $\left|\Theta_{\varphi_{3}}\right|=\sum_{\rho} \operatorname{deg}(\rho)^{2}([19], \S 2.4)$. Therefore we have that

$$
\begin{aligned}
& \operatorname{tr}\left(\overline{R_{\tilde{f}_{\Gamma}}}\right)=\sum_{\jmath, \rho} d_{j, \rho} \operatorname{tr}\left(\psi_{j, \rho}\left(\tilde{f}_{\Gamma}\right)\right) \\
& =\sum_{j \in J} \sum_{\rho} m_{j} \operatorname{deg}(\rho)^{2} \sum_{k=1}^{m_{j}} \widehat{f_{\mathrm{I}}}\left(\theta_{k}^{-1} \cdot \varphi_{j}^{-1}\right) \\
& =\sum_{j \in J} m_{j}\left(\sum_{\rho} \operatorname{deg}(\rho)^{2}\right) \sum_{k=1}^{m_{j}} \widehat{\widetilde{f_{\mathrm{I}}}}\left(\theta_{k}^{-1} \cdot \varphi_{j}^{-1}\right) \\
& =\sum_{j \in J} m_{j}\left|\Theta_{\varphi_{j}}\right| \sum_{k=1}^{m_{j}} \widehat{\widetilde{f_{\mathrm{r}}}}\left(\theta_{k}^{-1} \cdot \varphi_{J}^{-1}\right) \\
& =\sum_{\jmath \in J}|\Theta| \sum_{k=1}^{m_{j}} \widehat{\tilde{f}_{\mathrm{I}}}\left(\theta_{k}^{-1} \cdot \varphi_{j}^{-1}\right) \\
& =|\Theta| \sum_{\varphi \in \widehat{G / \Gamma}} \widehat{\widetilde{f}}_{\mathrm{I}}(\varphi) \\
& =|\Theta| \tilde{f}_{\Gamma}\left(\Gamma, e_{\Theta}\right)
\end{aligned}
$$

by the Poisson summation formula.

For $f \in C_{c}(G \rtimes \Theta) * C_{c}(G \rtimes \Theta)$, we can use the function $\tilde{f}$ to get a simplified version our extended Poisson Summation formula given by Corollary 7.3.4. This version no longer has any dependence on $\Theta$ on the spectral side. 
Corollary 7.4.6. For $f \in C_{c}(G \rtimes \Theta) * C_{c}(G \rtimes \Theta)$, we have

$$
|\Theta| \sum_{\gamma \in \Gamma} f\left(\gamma, e_{\Theta}\right)=\sum_{\jmath, \rho} d_{\jmath, \rho} \int_{G / \Gamma} f_{\Gamma}\left(x \Gamma, e_{\Theta}\right) \operatorname{tr}\left(\psi_{\jmath, \rho}\left(x \Gamma, e_{\Theta}\right)\right) d(x \Gamma)
$$

where the sum on the right is taken over all equivalence classes of irreducible representations of $G / \Gamma \rtimes \Theta$, and is absolutely convergent.

Proof: Suppose $f \in C_{c}(G \rtimes \Theta) * C_{c}(G \rtimes \Theta)$. We have

$$
\begin{aligned}
|\Theta| \sum_{\gamma \in \Gamma} f\left(\gamma, e_{\Theta}\right) & =|\Theta| f_{\Gamma}\left(e \Gamma, e_{\Theta}\right) \\
& =|\Theta| \tilde{f}_{\Gamma}\left(e \Gamma, e_{\Theta}\right) \\
& =\sum_{\jmath, \rho} d_{\jmath, \rho} \operatorname{tr}\left(\psi_{\jmath, \rho}\left(\tilde{f}_{\Gamma}\right)\right) \\
& =\sum_{\jmath, \rho} d_{\jmath, \rho} \int_{G / \Gamma \rtimes \Theta} \tilde{f}_{\Gamma}(x \Gamma, \theta) \operatorname{tr}\left(\psi_{\jmath, \rho}(x \Gamma, \theta)\right) d(x \Gamma, \theta) \\
& =\sum_{\jmath, \rho} d_{\jmath, \rho} \int_{G / \Gamma} \tilde{f}_{\Gamma}\left(x \Gamma, e_{\Theta}\right) \operatorname{tr}\left(\psi_{\jmath, \rho}\left(x \Gamma, e_{\Theta}\right)\right) d(x \Gamma) \\
& =\sum_{\jmath, \rho} d_{\jmath, \rho} \int_{G / \Gamma} f_{\Gamma}\left(x \Gamma, e_{\Theta}\right) \operatorname{tr}\left(\psi_{\jmath, \rho}\left(x \Gamma, e_{\Theta}\right)\right) d(x \Gamma) .
\end{aligned}
$$

\subsection{An Example on $\mathbb{Z}_{3}$}

Example: We return to the example from $\S 6.5$ where $G=\mathbb{Z}$ and $\Gamma=3 \mathbb{Z}$, so that $G / \Gamma \cong \mathbb{Z}_{3}$. To simplify notation, we will identify elements $m+\mathbb{Z}$ of $\mathbb{Z} / 3 \mathbb{Z}$ as elements $\bar{m}$ from $\mathbb{Z}_{3}$. Let $\mu_{\mathbb{Z}_{3}}$ be the normalized counting measure on $G / \Gamma=\mathbb{Z}_{3}$, meaning that 
for all $A \subseteq \mathbb{Z}_{3}$

$$
\mu_{\mathbb{Z}_{3}}(A)=\frac{|A|}{3}
$$

Let $\mu_{Z}$ be the counting measure on $\mathbb{Z}$ which is also rescaled by a factor of $1 / 3$. It can be shown that $\mu_{\mathbb{Z}_{3}}$ is the quotient measure on $G / \Gamma$ with respect to $\mu_{Z}$, which was discussed in $\S 3.1$. In $\S 2.2$ we saw that the group of irreducible characters on $\mathbb{Z}_{3}$ is the set $\widehat{\mathbb{Z}_{3}}=\left\{\varphi_{0}, \varphi_{1}, \varphi_{2}\right\}$, where $\varphi_{k}(\bar{m})=e^{\frac{2 k \pi m m}{3}}$. Let $\Theta=A u t(\mathbb{Z})=\{i d, \theta\}$, where $i d$ is the identity automorphism and $\theta$ is the automorphism $\theta(m)=-m$.

In $\S 6.5$, we determined that the complete set inequivalent irreducible representations for $\mathbb{Z}_{3} \rtimes \Theta$ is $\psi_{0, \rho_{0}}, \psi_{0, \rho_{1}}$ and $\psi_{1,1}$, where specifically $\psi_{0, \rho_{0}}$ is the trivial character, $\psi_{0, \rho_{1}}$ is the sign character given by $\psi_{0, \rho_{1}}(\bar{m}, \tau)=\rho_{1}(\tau)$ for all $\bar{m} \in \mathbb{Z}_{3}$, and $\psi_{1,1}=\operatorname{Ind} d_{\mathbb{Z}_{3}}^{\mathbb{Z}_{3} \rtimes \Theta} \varphi_{1} \otimes 1$ is a two-dimensional representation with trace values determined in $\S 6.5$ to be

$$
\begin{gathered}
\operatorname{tr}\left(\psi_{1, \mathbf{1}}(\bar{m}, \theta)\right)=0 \\
\operatorname{tr}\left(\psi_{1, \mathbf{1}}(\bar{m}, i d)\right)=\varphi_{1}(\bar{m})+\varphi_{1}(-\bar{m})=e^{\frac{2 \pi i m}{3}}+e^{\frac{4 \pi m m}{3}} .
\end{gathered}
$$

Suppose $f \in C_{c}(\mathbb{Z} \rtimes \Theta) * C_{c}(\mathbb{Z} \rtimes \Theta)$. By Proposition 7.1.1, we have that

$$
\begin{aligned}
\operatorname{tr}\left(\psi_{0, \rho_{0}}\left(f_{\Gamma}\right)\right) & =\int_{\mathbb{Z}_{3} \rtimes \Theta} f_{\Gamma}(\bar{m}, \tau) \operatorname{tr}\left(\psi_{0, \rho_{0}}(\bar{m}, \tau)\right) d(\bar{m}, \tau) \\
& =\int_{\mathbb{Z}_{3}} f_{\Gamma}(\bar{m}, i d) d(\bar{m})+\int_{\mathbb{Z}_{3}} f_{\Gamma}(\bar{m}, \theta) d(\bar{m}) \\
& =\frac{1}{3} \sum_{m=0}^{2} f_{\Gamma}(\bar{m}, i d)+\frac{1}{3} \sum_{m=0}^{2} f_{\Gamma}(\bar{m}, \theta) . \\
\operatorname{tr}\left(\psi_{0, \rho_{1}}\left(f_{\Gamma}\right)\right) & =\int_{\mathbb{Z}_{3} \rtimes \Theta} f_{\Gamma}(\bar{m}, \tau) \operatorname{tr}\left(\psi_{0, \rho_{1}}(\bar{m}, \tau)\right) d(\bar{m}, \tau) \\
& =\frac{1}{3} \sum_{m=0}^{2} f_{\Gamma}(\bar{m}, i d)+\frac{1}{3}\left(-\sum_{m=0}^{2} f_{\Gamma}(\bar{m}, \theta)\right) .
\end{aligned}
$$




$$
\begin{aligned}
\operatorname{tr}\left(\psi_{1,1}\left(f_{\Gamma}\right)\right) & =\int_{\mathbb{Z}_{3} \rtimes \Theta} f_{\Gamma}(\bar{m}, \tau) \operatorname{tr}\left(\psi_{1, \mathbf{1}}(\bar{m}, \tau)\right) d(\bar{m}, \tau) \\
& =\frac{1}{3} \sum_{m=0}^{2} f_{\Gamma}(\bar{m}, i d)\left(e^{\frac{2 \pi \imath m}{3}}+e^{\frac{4 \pi \imath m}{3}}\right) .
\end{aligned}
$$

Therefore, by Theorem 7.3.3, we have

$$
\begin{aligned}
\operatorname{tr}\left(\overline{R_{f_{\Gamma}}}\right) & =\operatorname{tr}\left(\psi_{0, \rho_{0}}\left(f_{\Gamma}\right)\right)+\operatorname{tr}\left(\psi_{0, \rho_{1}}\left(f_{\Gamma}\right)\right)+2 \operatorname{tr}\left(\psi_{\mathbf{1 , 1}}\left(f_{\Gamma}\right)\right) \\
& =\left(\frac{1}{3} \sum_{m=0}^{2} f_{\Gamma}(\bar{m}, i d)+\frac{1}{3} \sum_{m=0}^{2} f_{\Gamma}(\bar{m}, \theta)\right)+\left(\frac{1}{3} \sum_{m=0}^{2} f_{\Gamma}(\bar{m}, i d)+\frac{1}{3}\left(-\sum_{m=0}^{2} f_{\Gamma}(\bar{m}, \theta)\right)\right) \\
& +2\left(\frac{1}{3} \sum_{m=0}^{2} f_{\Gamma}(\bar{m}, i d)\left(e^{\frac{2 \pi i m}{3}}+e^{\frac{4 \pi i m}{3}}\right)\right) \\
& =\sum_{m=0}^{2}\left(\frac{2}{3} f_{\Gamma}(\bar{m}, i d)+\frac{1}{3} f_{\Gamma}(\bar{m}, \theta)-\frac{1}{3} f_{\Gamma}(\bar{m}, \theta)+\frac{2}{3} f_{\Gamma}(\bar{m}, i d)\left(e^{\frac{2 \pi i m}{3}}+e^{\frac{4 \pi m m}{3}}\right)\right) \\
& =\frac{2}{3} \sum_{m=0}^{2} f_{\Gamma}(\bar{m}, i d)\left(1+e^{\frac{2 \pi i m}{3}}+e^{\frac{4 \pi i m}{3}}\right) .
\end{aligned}
$$

When $m=1$ or $m=2$, the value of $1+e^{\frac{2 \pi \imath m}{3}}+e^{\frac{4 \pi \imath m}{3}}$ is 0 . Therefore, we get that

$$
\begin{aligned}
\operatorname{tr}\left(\overline{R_{f_{\Gamma}}}\right) & =\frac{2}{3} \sum_{m=0}^{2} f_{\Gamma}(\bar{m}, i d)\left(1+e^{\frac{2 \pi \imath m}{3}}+e^{\frac{4 \pi \imath m}{3}}\right) \\
& =\frac{2}{3} f_{\Gamma}(\overline{0}, i d)\left(1+e^{0}+e^{0}\right) \\
& =2 f_{\Gamma}(\overline{0}, i d) .
\end{aligned}
$$

This, as expected, gives the same result as the formula given in Theorem 5.5.2

$$
\operatorname{tr}\left(\overline{R_{f_{\Gamma}}}\right)=|\Theta| f_{\Gamma}(\overline{0}, i d)=2 f_{\Gamma}(\overline{0}, i d)
$$

Note that there is some cancellation between the trace values of the linear operators $\psi_{0, \rho_{0}}\left(f_{\Gamma}\right)$ and $\psi_{0, \rho_{1}}\left(f_{\Gamma}\right)$ with the value $\frac{1}{3} \sum_{m=0}^{2} f_{\Gamma}(\bar{m}, \theta)$. 


\section{Chapter 8}

\section{Conclusion}

In the example from $\S 7.5$, we see that the values of $\operatorname{tr}\left(\psi_{0, \rho_{0}}\left(f_{\Gamma}\right)\right)$ and $\operatorname{tr}\left(\psi_{0, \rho_{1}}\left(f_{\Gamma}\right)\right)$ have some cancellation. These representations $\psi_{0, \rho_{0}}$ and $\psi_{0, \rho_{1}}$ are the representations of $G / \Gamma \rtimes \Theta$ which are given by extension (as opposed to induction) from $G / \Gamma$ to $G / \Gamma \rtimes \Theta$. Consider generalizing our methods to the case of $H=\left(G L\left(2, \mathbb{A}_{\mathbb{F}}\right) \rtimes\right.$ $\operatorname{Gal}(\mathbb{F} / \mathbb{Q})) / G L(2, \mathbb{F})$ mentioned in the introduction. In that example, there are representations of $H$ which also extend from $G L\left(2, \mathbb{A}_{\mathbb{F}}\right) / G L(2, \mathbb{F})$ to $H$ and one would like to find a trace formula on $H$ in which no cancellation in the traces of these representations occurs. Perhaps, through analysis of the present formula, one may be inspired to alter it so as to make this possible. 


\section{Bibliography}

[1] M. Artin, Algebra, Second Edition, Pearson Education, Inc., 2011.

[2] B. Bollobas, Linear Analysis, Cambridge University Press, 1999.

[3] D. Cohn, Measure Theory, Birkhauser, Boston, Massachusetts, 1980.

[4] M. Duflo, Généralités sur les Représentations Induites, Représentations des Groupes de Lie Résolubles, Monographies de la Soc. Math. de France volume 4 (1972).

[5] D. Dummit and R. Foote, Abstract Algebra, Third Edition, John Wiley and Sons, Inc., 2004.

[6] R.E. Edwards, Functional Analysis. Theory and Applications, Hlt, Rinehart and Winston, 1965.

[7] G. Folland, A Course in Abstract Harmonic Analysis, CRC Press LLC, 1995.

[8] E. Hewitt and K. Ross., Abstract Harmonic Analysis, Vol. I, Springer-Verlag, 1963.

[9] E. Hewitt and K. Ross, Abstract Harmonic Analysis, Vol. II, Springer-Verlag, 1963.

[10] E. Hewitt and K. Stromberg, Real and Abstract Analysis, Springer-Verlag, 1965.

[11] D. Hilbert and R. Courant, Methoden der mathematischen Physik, Springer-Verlag, Berlin, 1924.

[12] N. Jacobian, Basic Algebra II, 2nd Edition, Freeman and Co., 1989.

[13] S. Lang, Complex Analysis, Springer-Verlag, 1985.

[14] R.P. Langlands, Base Change for GL(2), Annals of Mathematics Studies, 96, Princeton University Press, 1980.

[15] L. Nachbin, The Haar Integral, Robert E. Krieger Publishing Company, Huntington, New York, 1976.

[16] S.D. Poisson, Sur la distribution de la chaleur dans les corps solides, Journal d'Ecole Polytechnique (1823).

[17] W. Rudin, Functional Analysis, McGraw-Hill Book Company, 1973.

[18] D. Serre, Matrices. Theories and Applications, Springer-Verlag, 2002.

[19] J.P. Serre, Linear Representations of Finite Groups, Springer-Verlag, 1977.

[20] B. Simon, Representations of Finite and Compact Groups, American Mathematical Society, 1996.

[21] B. Simon and M. Reed, Methods of Modern Mathematical Physics I: Functional Analysis, Academic Press, 1972.

[22] E.M. Stein, Fourier Analysis. An Introduction, Princeton University Press, 2003.

[23] S. Willard, General Topology, Dover Publications, Inc., Mineola, New York, 1998. 